\title{
An introduction to Total Variation for Image Analysis
}

\author{
A. Chambolle*, V. Caselles; M. Novaga $\ddagger$ \\ D. Cremers ${ }^{\S}$ and T. Pock
}

\begin{abstract}
These notes address various theoretical and practical topics related to Total Variation-based image reconstruction. They focuse first on some theoretical results on functions which minimize the total variation, and in a second part, describe a few standard and less standard algorithms to minimize the total variation in a finite-differences setting, with a series of applications from simple denoising to stereo, or deconvolution issues, and even more exotic uses like the minimization of minimal partition problems.
\end{abstract}

\footnotetext{
${ }^{*}$ CMAP, Ecole Polytechnique, CNRS, 91128, Palaiseau, France. e-mail: antonin.chambolle@polytechnique.fr

${ }^{\dagger}$ Departament de Tecnologia, Universitat Pompeu-Fabra, Barcelona, Spain. e-mail: vicent.caselles@tecn.upf.es

${ }^{\ddagger}$ Dipartimento di Matematica, Università di Padova, Via Trieste 63, 35121 Padova, Italy. email: novaga@math.unipd.it

${ }^{\S}$ Department of Computer Science, University of Bonn, Römerstraße 164, 53117 Bonn, Germany. e-mail: dcremers@cs.uni-bonn.de

`Institute for Computer Graphics and Vision, Graz University of Technology, 8010 Graz, Austria. e-mail pock@icg.tugraz.at
} 


\section{Contents}

1 The total variation 4

1.1 Why is the total variation useful for images? . . . . . . . . . . . . 4

1.1.1 The Bayesian approach to image reconstruction . . . . . . . . 4

1.1.2 Variational models in the continuous setting . . . . . . . . 6

1.1.3 A convex, yet edge-preserving approach . . . . . . . . . 9

1.2 Some theoretical facts: definitions, properties . . . . . . . . . . 12

1.2 .1 Definition . . . . . . . . . . . . . . . . 12

1.2 .2 An equivalent definition $(*) \ldots \ldots \ldots \ldots$

1.2.3 Main properties of the total variation . . . . . . . . . 13

1.2.4 Functions with bounded variation . . . . . . . . . 15

1.3 The perimeter. Sets with finite perimeter . . . . . . . . . . . . 19

1.3.1 Definition, and an inequality . . . . . . . . . . . 19

1.3.2 The reduced boundary, and a generalization of Green's formula 19

1.3.3 The isoperimetric inequality . . . . . . . . . . . . . . 21

1.4 The co-area formula . . . . . . . . . . . . . . . . . . 21

1.5 The derivative of a $B V$ function $(*) \ldots \ldots \ldots \ldots . \ldots \ldots$

2 Some functionals where the total variation appears 25

2.1 Perimeter minimization . . . . . . . . . . . . . . . 25

2.2 The Rudin-Osher-Fatemi problem . . . . . . . . . . . . . 26

2.2.1 The Euler-Lagrange equation . . . . . . . . . . . . . 27

2.2.2 The problem solved by the level sets . . . . . . . . . . 30

2.2 .3 A few explicit solutions . . . . . . . . . . . . . 34

2.2 .4 The discontinuity set $\ldots \ldots \ldots \ldots \ldots$

2.2 .5 Regularity . . . . . . . . . . . . . . . . 39

3 Algorithmic issues $\quad 40$

3.1 Discrete problem . . . . . . . . . . . . . . . . . . 40

3.2 Basic convex analysis - Duality . . . . . . . . . . . . . . . 42

3.2.1 Convex functions - Legendre-Fenchel conjugate . . . . . . . . 42

3.2 .2 Subgradient . . . . . . . . . . . . . . . . . 45

3.2 .3 The dual of $(R O F) \ldots \ldots \ldots \ldots \ldots$. . . . . . . . . 47

3.2 .4 "Proximal" operator . . . . . . . . . . . . . . 48

3.3 Gradient descent . . . . . . . . . . . . . . . . . . . . . . . . 49 
3.3.1 Splitting, and Projected gradient descent . . . . . . . . . 50

3.3.2 Improvements: optimal first-order methods . . . . . . . . . . 53

3.4 Augmented Lagrangian approaches . . . . . . . . . . . . . . . 54

3.5 Primal-dual approaches . . . . . . . . . . . . . . . . . . . 54

3.6 Graph-cut techniques . . . . . . . . . . . . . . . . . 57

3.7 Comparisons of the numerical algorithms . . . . . . . . . 58

4 Applications $\quad 62$

4.1 Total variation based image deblurring and zooming . . . . . . . . 62

4.2 Total variation with $L^{1}$ data fidelity term . . . . . . . . . . . . 63

4.3 Variational models with possibly nonconvex data terms . . . . . . 64

4.3.1 Convex representation . . . . . . . . . . . . . . 64

4.3 .2 Convex relaxation . . . . . . . . . . . . . . 69

4.3 .3 Numerical resolution . . . . . . . . . . . . . . . . . . 70

4.4 The minimal partition problem . . . . . . . . . . . 73

$\begin{array}{ll}\text { A A proof of convergence } & 77\end{array}$ 


\section{Introduction}

These lecture notes have been prepared for the summer school on sparsity organized in Linz, Austria, by Massimo Fornasier and Ronny Romlau, during the week Aug. 31st-Sept. 4th, 2009. They contain work done in collaboration with Vicent Caselles and Matteo Novaga (for the first theoretical parts), and with Thomas Pock and Daniel Cremers, for the algorithmic parts. All are obviously warmly thanked for the work we have done together so far - and the work still to be done! I thank particularly Thomas Pock for pointing out very recent references on primal-dual algorithms and help me clarify the jungle of algorithms.

I also thank, obviously, the organizers of the summer school for inviting me to give these lectures. It was a wonderful scientific event, and we had a great time in Linz.

Antonin Chambolle, nov. 2009

\section{The total variation}

\subsection{Why is the total variation useful for images?}

The total variation has been introduced for image denoising and reconstruction in a celebrated paper of 1992 by Rudin, Osher and Fatemi [68]. Let us discuss how such a model, as well as other variational approaches for image analysis problems, arise in the context of Bayesian inference.

\subsubsection{The Bayesian approach to image reconstruction}

Let us first consider the discrete setting, where images $g=\left(g_{i, j}\right)_{1 \leq i, j \leq N}$ are discrete, bounded $\left(g_{i, j} \in[0,1]\right.$ or $\left.\{0, \ldots, 255\}\right)$ 2D-signals. The general idea for solving (linear) inverse problems is to consider

- A model: $g=A u+n-u \in \mathbb{R}^{N \times N}$ is the initial "perfect" signal, $A$ is some transformation (blurring, sampling, or more generally some linear operator, like a Radon transform for tomography). $n=\left(n_{i, j}\right)$ is the noise: in the simplest situations, we consider a Gaussian norm with average 0 and standard deviation $\sigma$. 
- An a priori probability density for "perfect" original signals, $P(u) \sim e^{-p(u)} d u$. It represents the idea we have of perfect data (in other words, the model for the data).

Then, the a posteriori probability for $u$ knowing $g$ is computed from Bayes' rule, which is written as follows:

$$
P(u \mid g) P(g)=P(g \mid u) P(u)
$$

Since the density for the probability of $g$ knowing $u$ is the density for $n=g-A u$, it is

$$
e^{-\frac{1}{2 \sigma^{2}} \sum_{i, j}\left|g_{i, j}-(A u)_{i, j}\right|^{2}}
$$

and we deduce from $(B R)$ that the density for $P(u \mid g)$, the probability of $u$ knowing the observation $g$ is

$$
\frac{1}{Z(g)} e^{-p(u)} e^{-\frac{1}{2 \sigma^{2}} \sum_{i, j}\left|g_{i, j}-(A u)_{i, j}\right|^{2}}
$$

with $Z(g)$ a renormalization factor which is simply

$$
Z(g)=\int_{u} e^{-\left(p(u)+\frac{1}{2 \sigma^{2}} \sum_{i, j}\left|g_{i, j}-(A u)_{i, j}\right|^{2}\right)} d u
$$

(the integral is on all possible images $u$, that is, $\mathbb{R}^{N \times N}$, or $[0,1]^{N \times N} \ldots$ ).

The idea of "maximum a posteriori" (MAP) image reconstruction is to find the "best" image as the one which maximizes this probability, or equivalently, which solves the minimum problem

$$
\min _{u} p(u)+\frac{1}{2 \sigma^{2}} \sum_{i, j}\left|g_{i, j}-(A u)_{i, j}\right|^{2} .
$$

Let us observe that this is not necessarily a good idea, indeed, even if our model is perfectly well built, the image with highest probability given by the resolution of $(M A P)$ might be very rare. Consider for instance figure 1 where we have plotted a (of course quite strange) density on $[0,1]$, whose maximum is reached at $x=1 / 20$, while, in fact, the probability that $x \in[0,1 / 10]$ is $1 / 10$, while the probability $x \in[1 / 10,1]$ is $9 / 10$. In particular the expectation of $x$ is $1 / 2$. This shows that it might make more sense to try to compute the expectation of $u$ (given $g$ )

$$
E(u \mid g)=\frac{1}{Z(g)} \int_{u} u e^{-\left(p(u)+\frac{1}{2 \sigma^{2}} \sum_{i, j}\left|g_{i, j}-(A u)_{i, j}\right|^{2}\right)} d u .
$$

However, such a computation is hardly tractable in practice, and requires subtle algorithms based on complex stochastic techniques (Monte Carlo methods with 


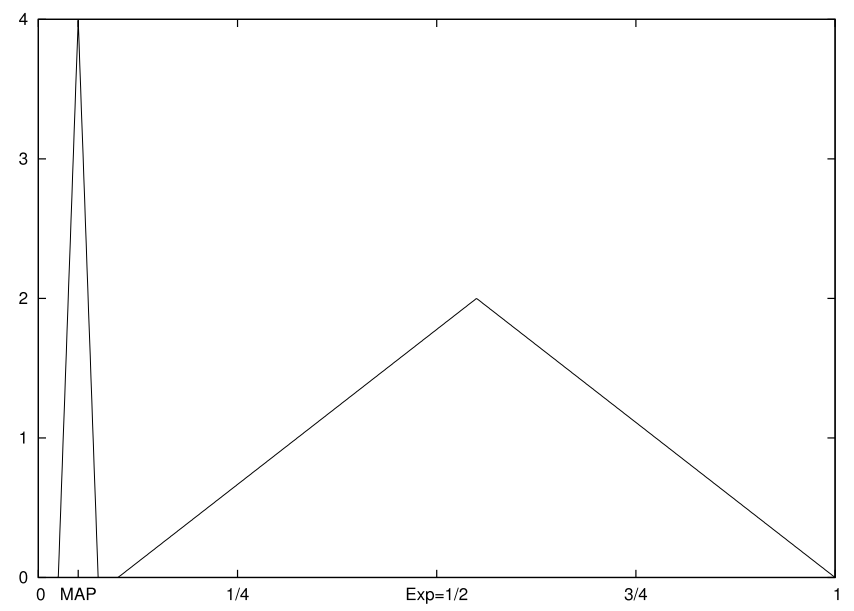

Figure 1: A strange probability density

Markov Chains, or MCMC). These approaches seem yet not efficient enough for complex reconstruction problems. See for instance $[49,66]$ for experiments in this direction.

\subsubsection{Variational models in the continuous setting}

Now, let us forget the Bayesian, discrete model and just retain to simplify the idea of minimizing an energy such as in $(M A P)$. We will now write our images in the continuous setting: as grey-level values functions $g, u: \Omega \mapsto \mathbb{R}$ or $[0,1]$, where $\Omega \subset \mathbb{R}^{2}$ will in practice be (most of the times) the square $[0,1]^{2}$, but in general any (bounded) open set of $\mathbb{R}^{2}$, or more generally $\mathbb{R}^{N}, N \geq 1$.

The operator $A$ will be a bounded, linear operator (for instance from $L^{2}(\Omega)$ to itself), but from now on, to simplify, we will simply consider $A=I d$ (the identity operator $A u=u$ ), and return to more general (and useful) cases in the Section 3 on numerical algorithms.

In this case, the minimization problem $(M A P)$ can be written

$$
\min _{u \in L^{2}(\Omega)} \lambda F(u)+\frac{1}{2} \int_{\Omega}|u(x)-g(x)|^{2} d x
$$

where $F$ is a functional corresponding to the a priori probability density $p(u)$, and which synthetises the idea we have of the type of signal we want to recover, and $\lambda>0$ a weight balancing the respective importance of the two terms in the problem. We consider $u$ in the space $L^{2}(\Omega)$ of functions which are square-integrable, since 
the energy will be infinite if $u$ is not, this might not always be the right choice (with for instance general operators $A$ ).

Now, what is the good choice for $F$ ? Standard Tychonov regularization approaches will usually consider quadratic $F$ 's, such as $F(u)=\frac{1}{2} \int_{\Omega} u^{2} d x$ or $\frac{1}{2} \int_{\Omega}|\nabla u|^{2} d x$. In this last expression,

$$
\nabla u(x)=\left(\begin{array}{c}
\frac{\partial u}{\partial x_{1}}(x) \\
\vdots \\
\frac{\partial u}{\partial x_{N}}(x)
\end{array}\right)
$$

is the gradient of $u$ at $x$. The advantage of these choices is that the corresponding problem to solve is linear, indeed, the Euler-Lagrange equation for the minimization problem is, in the first case,

$$
\lambda u+u-g=0,
$$

and in the second,

$$
-\lambda \Delta u+u-g=0,
$$

where $\Delta u=\sum_{i} \partial^{2} u / \partial x_{i}^{2}$ is the Laplacian of $u$. Now look at Fig. 2: in the first
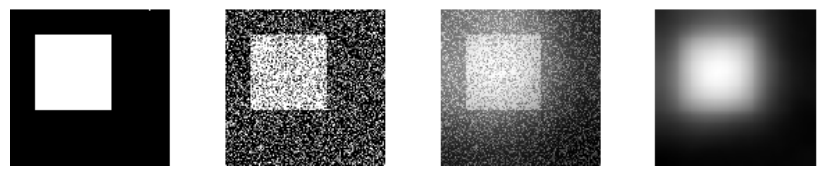

Figure 2: A white square on a dark background, then with noise, then restored with $F=\frac{1}{2} \int|u|^{2}$, then with $F=\frac{1}{2} \int|\nabla u|^{2}$

case, no regularization has occured. This is simply because $F(u)=\frac{1}{2} \int u^{2}$ enforces no spatial regularization of any kind. Of course, this is a wrong choice, since all "natural" images show a lot of spatial regularity. On the other hand, in the second case, there is too much spatial regularization. Indeed, the image $u$ must belong to the space $H^{1}(\Omega)$ of functions whose derivative is square-integrable. However, it is well-known that such functions cannot present discontinuities across hypersurfaces, that is, in 2 dimension, across lines (such as the edges or boundaries of objects in an image).

A quick argument to justify this is as follows. Assume first $u:[0,1] \rightarrow \mathbb{R}$ is a 1-dimensional function which belongs to $H^{1}(0,1)$. Then for each $0<s<t<1$,

$$
u(t)-u(s)=\int_{s}^{t} u^{\prime}(r) d r \leq \sqrt{t-s} \sqrt{\int_{s}^{t}\left|u^{\prime}(r)\right|^{2} d r} \leq \sqrt{t-s}\|u\|_{H^{1}}^{2}
$$


so that $u$ must be $1 / 2$-Hölder continuous (and in fact a bit better). (This computation is a bit formal, it needs to be performed on smooth functions and is then justified by density for any function of $H^{1}(0,1)$.)

Now if $u \in H^{1}((0,1) \times(0,1))$, one can check that for a.e. $y \in(0,1), x \mapsto$ $u(x, y) \in H^{1}(0,1)$, which essentially comes from the fact that

$$
\int_{0}^{1}\left(\int_{0}^{1}\left|\frac{\partial u}{\partial x}(x, y)\right|^{2} d x\right) d y \leq\|u\|_{H^{1}}^{2}<+\infty .
$$

It means that for a.e. $y, x \mapsto u(x, y)$ will be $1 / 2$-Hölder continuous in $x$, so that it certainly cannot jump across the vertical boundaries of the square in Fig. 2. In fact, a similar kind of regularity can be shown for any $u \in W^{1, p}(\Omega)$, $1 \leq p \leq+\infty$ (although for $p=1$ it is a bit weaker, but still "large" discontinuities are forbidden), so that replacing $\int_{\Omega}|\nabla u|^{2} d x$ with $\int_{\Omega}|\nabla u|^{p} d x$ for some other choice of $p$ should not produce any better result. We will soon check that the reality is a bit more complicated.

So what is a good " $F(u)$ " for images? There have been essentially two types of answers, during the 80's and early 90's, to this question. As we have checked, a good $F$ should simultaneously ensure some spatial regularity, but also preserve the edges. The first idea in this direction is due to D. Geman and S. Geman [37], where it is described in the Bayesian context. They consider an additional variable $\ell=\left(\ell_{i+1 / 2, j}, \ell_{i, j+1 / 2}\right)_{i, j}$ which can take only values 0 and $1: \ell_{i+1 / 2, j}=1$ means that there is an edge between the locations $(i, j)$ and $(i+1, j)$, while 0 means that there is no edge. Then, $p(u)$ in the a priori probability density of $u$ needs to be replaced with $p(u, \ell)$, which typically will be of the form

$$
\begin{array}{r}
p(u, \ell)=\lambda \sum_{i, j}\left(\left(1-\ell_{i+\frac{1}{2}, j}\right)\left(u_{i+1, j}-u_{i, j}\right)^{2}+\left(1-\ell_{i, j+\frac{1}{2}}\right)\left(u_{i, j+1}-u_{i, j}\right)^{2}\right) \\
+\mu \sum_{i, j}\left(\ell_{i+\frac{1}{2}, j}+\ell_{i, j+\frac{1}{2}}\right),
\end{array}
$$

with $\lambda, \mu$ positive parameters. Hence, the problem $(M A P)$ will now look like (taking as before $A=I d)$ :

$$
\min _{u, \ell} p(u, \ell)+\frac{1}{2 \sigma^{2}} \sum_{i, j}\left|g_{i, j}-u_{i, j}\right|^{2}
$$

In the continuous setting, it has been observed by D. Mumford and J. Shah [56] that the set $\{\ell=1\}$ could be considered as a $1-$ dimensional curve $K \subset \Omega$, while 
the way it was penalized in the energy was essentially proportional to its length. So that they proposed to consider the minimal problem

$$
\min _{u, K} \lambda \int_{\Omega \backslash K}|\nabla u|^{2} d x+\mu \text { length }(K)+\int_{\Omega}|u-g|^{2} d x
$$

among all 1-dimensional closed subsets $K$ of $\Omega$ and all $u \in H^{1}(\Omega \backslash K)$. This is the famous "Mumford-Shah" functional whose study has generated a lot of interesting mathematical tools and problems in the past 20 years, see in particular $[54,7,29$, $51]$.

However, besides being particularly difficult to analyse mathematically, this approach is also very complicated numerically since it requires to solve a nonconvex problem, and there is (except in a few particular situations) no way, in general, to know whether a candidate is really a minimizer. The most efficient methods rely either on stochastic algorithms [55], or on variational approximations by " $\Gamma$-convergence", see $[8,9]$ solved by alternate minimizations. The exception is the one-dimensional setting where a dynamical programming principle is available and an exact solution can be computed in polynomial time.

\subsubsection{A convex, yet edge-preserving approach}

In the context of image reconstruction, it was proposed first by Rudin, Osher and Fatemi in [68] to consider the "Total Variation" as a regularizer $F(u)$ for $(M A P c)$. The precise definition will be introduced in the next section. It can be seen as an extension of the energy

$$
F(u)=\int_{\Omega}|\nabla u(x)| d x
$$

well defined for $C^{1}$ functions, and more generally for functions $u$ in the Sobolev space $W^{1,1}$. The big advantage of considering such a $F$ is that it is now convex in the variable $u$, so that the problem $(M A P c)$ will now be convex and many tools from convex optimization can be used to tackle it, with a great chance of success (see Definition 3.2 and Section 3). However, as we have mentioned before, a function in $W^{1,1}$ cannot present a discontinuity accross a line (in 2D) or a hypersurface (in general). Exactly as for $H^{1}$ functions, the idea is that if $u \in W^{1,1}(0,1)$ and $0<s<t<1$,

$$
u(t)-u(s)=\int_{s}^{t} u^{\prime}(r) d r \leq \int_{s}^{t}\left|u^{\prime}(r)\right| d r
$$

and if $u^{\prime} \in L^{1}(0,1)$, the last integral must vanish as $|t-s| \rightarrow 0$ (and, even, in fact, uniformly in $s, t$ ). We deduce that $u$ is (uniformly) continuous on $[0,1]$, and, 
as before, if now $u \in W^{1,1}((0,1) \times(0,1))$ is an image in $2 \mathrm{D}$, we will have that for a.e. $y \in(0,1), u(\cdot, y)$ is a $1 \mathrm{D} W^{1,1}$ function hence continuous in the variable $x$.

But what happens when one tries to resolve

$$
\min _{u} \lambda \int_{0}^{1}\left|u^{\prime}(t)\right| d t+\int_{0}^{1}|u(t)-g(t)|^{2} d t ?
$$

Consider the simple case where $g=\chi_{(1 / 2,1)}$ (that is 0 for $t<1 / 2,1$ for $t>1 / 2$ ). First, there is a "maximum principle": if $u$ is a candidate (which we assume in $W^{1,1}(0,1)$, or to simplify continuous and piecewise $\left.C^{1}\right)$ for the minimization, then also $v=\min \{u, 1\}$ is. Moreover, $v^{\prime}=u^{\prime}$ whenever $u<1$ and $v^{\prime}=0$ a.e. on $\{v=1\}$, that is, where $u \geq 1$. So that clearly, $\int_{0}^{1}\left|v^{\prime}\right| \leq \int_{0}^{1}\left|u^{\prime}\right|$ (and the inequality is strict if $v \neq u$ ). Moreover, since $g \leq 1$, also $\int_{0}^{1}|v-g|^{2} \leq \int_{0}^{1}|u-g|^{2}$. Hence,

$$
\mathcal{E}(v):=\lambda \int_{0}^{1}\left|v^{\prime}(t)\right| d t+\int_{0}^{1}|v(t)-g(t)|^{2} d t \leq \mathcal{E}(u)
$$

(with a strict inequality if $v \neq u$ ). This tells us that a minimizer, if it exists, must be $\leq 1$ a.e. ( 1 here is the maximum value of $g$ ). In the same way, one checks that it must be $\geq 0$ a.e. (the minimum value of $g$ ). Hence we can restrict ourselves to functions between 0 and 1 .

Then, by symmetry, $t \mapsto 1-u(1-t)$ has the same energy as $u$, and by convexity,

$$
\mathcal{E}\left(\frac{1-u(1-\cdot)+u}{2}\right) \leq \frac{1}{2} \mathcal{E}(1-u(1-\cdot))+\frac{1}{2} \mathcal{E}(u)=\mathcal{E}(u)
$$

so that $v: t \mapsto(1-u(1-t)+u(t)) / 2$ has also lower energy (and again, one can show that the energy is "strictly" convex so that this is strict if $v \neq u$ ): but $v=u$ iff $u(1-t)=1-u(t)$, so that any solution must have this symmetry.

Let now $m=\min u=u(a)$, and $M=1-m=\max u=u(b)$ : it must be that (assuming for instance that $a<b$ )

$$
\int_{0}^{1}\left|u^{\prime}(t)\right| d t \geq \int_{a}^{b}\left|u^{\prime}(t)\right| d t \geq \int_{a}^{b} u^{\prime}(t) d t=M-m=1-2 m
$$

(and again all this is strict except when $u$ is nondecreasing, or nonincreasing).

To sum up, a minimizer $u$ of $\mathcal{E}$ should be between two values $0 \leq m \leq M=$ $1-m \leq 1$ (hence $m \in[0,1 / 2]$ ), and have the symmetry $u(1-t)=1-u(t)$. In particular, we should have

$$
\mathcal{E}(u) \geq \lambda(M-m)+\int_{0}^{\frac{1}{2}} m^{2}+\int_{\frac{1}{2}}^{1}(1-M)^{2}=\lambda(1-2 m)+m^{2}
$$


which is minimal for $m=\lambda>0$ provided $\lambda \leq 1 / 2$, and $m=1 / 2$ if $\lambda \geq 1 / 2$ (remember $m \in[0,1 / 2]$ ). In particular, in the latter case $\lambda \geq 1 / 2$, we deduce that the only possible minimizer is the function $u(t) \equiv 1 / 2$.

Assume then that $\lambda<1 / 2$, so that for any $u$,

$$
\mathcal{E}(u) \geq \lambda(1-\lambda)
$$

and consider for $n \geq 2, u_{n}(t)=\lambda$ if $t \in[0,1 / 2-1 / n], u_{n}(t)=1 / 2+n(t-1 / 2)(1 / 2-$ $\lambda)$ if $|t-1 / 2| \leq 1 / n$, and $1-\lambda$ if $t \geq 1 / 2+1 / n$. Then, since $u_{n}$ is nondecreasing, $\int_{0}^{1}\left|u^{\prime}\right|=\int_{0}^{1} u^{\prime}=1-2 \lambda$ so that

$$
\mathcal{E}\left(u_{n}\right) \leq \lambda(1-2 \lambda)+\left(1-\frac{2}{n}\right) \lambda^{2}+\frac{2}{n} \rightarrow \lambda(1-\lambda)
$$

as $n \rightarrow \infty$. Hence: $\inf _{u} \mathcal{E}(u)=\lambda(1-\lambda)$. Now, for a function $u$ to be a minimizer, we see that: it must be nondecreasing and grow from $\lambda$ to $1-\lambda$ (otherwise the term $\int_{0}^{1}\left|u^{\prime}\right|$ will be too large), and it must satisfy as well

$$
\int_{0}^{1}|u(t)-g(t)|^{2} d t=\lambda^{2}
$$

while from the first condition we deduce that $|u-g| \geq \lambda$ a.e.: hence we must have $|u-g|=\lambda$, that is, $u=\lambda$ on $[0,1 / 2)$ and $1-\lambda$ on $(1 / 2,1]$. But this $u$, which is actually the limit of our $u_{n}$ 's, is not differentiable: this shows that one must extend in an appropriate way the notion of derivative to give a solution to problem (1) of minimizing $\mathcal{E}$ : otherwise it cannot have a solution. In particular, we have seen that for all the functions $u_{n}, \int_{0}^{1}\left|u_{n}^{\prime}\right|=1-2 \lambda$, so that for our discontinuous limit $u$ it is reasonable to assume that $\int\left|u^{\prime}\right|$ makes sense. This is what we will soon define properly as the "total variation" of $u$, and we will see that it makes sense for a whole category of non necessarily continuous functions, namely, the "functions with bounded variation" (or $B V$ functions). Observe that we could define, in our case, for any $u \in L^{1}(0,1)$,

$$
F(u)=\inf \left\{\lim _{n \rightarrow \infty} \int_{0}^{1}\left|u_{n}^{\prime}(t)\right| d t: u_{n} \rightarrow u \text { in } L^{1}(0,1) \text { and } \lim _{n} \int_{0}^{1}\left|u_{n}^{\prime}\right| \text { exists. }\right\} .
$$

In this case, we could check easily that our discontinuous solution is the (unique) minimizer of

$$
\lambda F(u)+\int_{0}^{1}|u(t)-g(t)|^{2} d t .
$$


It turns out that this definition is consistent with the more classical definition of the total variation which we will introduce hereafter, in Definition 1.1 (see inequality (2) and Thm. 1).

What have we learned from this example? If we introduce, in Tychonov's regularization, the function $F(u)=\int_{\Omega}|\nabla u(x)| d x$ as a regularizer, then in general the problem $(M A P c)$ will have no solution in $W^{1,1}(\Omega)$ (where $F$ makes sense). But, there should be a way to appropriately extend $F$ to more general functions which can have (large) discontinuities and not be in $W^{1,1}$, so that $(M A P c)$ has a solution, and this solution can have edges! This was the motivation of Rudin, Osher and Fatemi [68] to introduce the Total Variation as a regularizer $F(u)$ for inverse problems of type $(M A P c)$. We will now introduce more precisely, from a mathematical point of view, this functional, and give its main properties.

\subsection{Some theoretical facts: definitions, properties}

The material in this part is mostly extracted from the textbooks $[40,74,34,7]$, which we invite the reader to consult for further details.

\subsubsection{Definition}

Definition 1.1. The total variation of an image is defined by duality: for $u \in$ $L_{\text {loc }}^{1}(\Omega)$ it is given by

$$
J(u)=\sup \left\{-\int_{\Omega} u \operatorname{div} \phi d x: \phi \in C_{c}^{\infty}\left(\Omega ; \mathbb{R}^{N}\right),|\phi(x)| \leq 1 \forall x \in \Omega\right\}
$$

A function is said to have Bounded Variation whenever $J(u)<+\infty$. Typical examples include:

- A smooth function $u \in C^{1}(\Omega)$ (or in fact a function $u \in W^{1,1}(\Omega)$ ): in this case,

$$
-\int_{\Omega} u \operatorname{div} \phi d x=\int_{\Omega} \phi \cdot \nabla u d x
$$

and the sup over all $\phi$ with $|\phi| \leq 1$ is $J(u)=\int_{\Omega}|\nabla u| d x$.

- The characteristic function of a set with smooth (or $C^{1,1}$ ) boundary: $u=\chi_{E}$, in this case

$$
-\int_{\Omega} u \operatorname{div} \phi d x=-\int_{\partial E} \phi \cdot \nu_{E} d \sigma
$$


and one can reach the sup (which corresponds to $\phi=-\nu_{E}$, the outer normal to $\partial E$, on $\partial E \cap \Omega$, while $\phi=0$ on $\partial E \cap \partial \Omega$ ) by smoothing, in a neighborhood of the boundary, the gradient of the signed distance function to the boundary. We obtain that $J(u)=\mathcal{H}^{N-1}(\partial E \cap \Omega)$, the perimeter of $E$ in $\Omega$.

Here, $\mathcal{H}^{N-1}(\cdot)$ is the $(N-1)$-dimensional Hausdorff measure, see for instance [35, $54,7]$ for details.

\subsubsection{An equivalent definition $(*)$}

It is well-known (see for instance [69]) that any $u \in L_{\text {loc }}^{1}(\Omega)$ defines a distribution

$$
\begin{aligned}
T_{u}: \mathcal{D}(\Omega) & \rightarrow \mathbb{R} \\
\phi & \mapsto \int_{\Omega} \phi(x) u(x) d x
\end{aligned}
$$

where here $\mathcal{D}(\Omega)$ is the space of smooth functions with compact support $\left(C_{c}^{\infty}(\Omega)\right)$ endowed with a particular topology, and $T_{u}$ is a continuous linear form on $\mathcal{D}(\Omega)$, that is, $T_{u} \in \mathcal{D}^{\prime}(\Omega)$. The derivative of $T_{u}$ is then defined as $(i=1, \ldots, N)$

$$
\left\langle\frac{\partial T_{u}}{\partial x_{i}}, \phi\right\rangle_{\mathcal{D}^{\prime}, \mathcal{D}}:=-\left\langle T_{u}, \frac{\partial \phi}{\partial x_{i}}\right\rangle_{\mathcal{D}^{\prime}, \mathcal{D}}=-\int_{\Omega} u(x) \frac{\partial \phi}{\partial x_{i}}(x) d x
$$

(which clearly extends the integration by parts: if $u$ is smooth, then $\partial T_{u} / \partial x_{i}=$ $\left.T_{\partial u / \partial x_{i}}\right)$. We denote by $D u$ the (vectorial) distribution $\left(\partial T_{u} / \partial x_{i}\right)_{i=1}^{N}$.

Then, if $J(u)<+\infty$, it means that for all vector field $\phi \in C_{c}^{\infty}\left(\Omega ; \mathbb{R}^{N}\right)$

$$
\langle D u, \phi\rangle_{\mathcal{D}^{\prime}, \mathcal{D}} \leq J(u) \sup _{x \in \Omega}|\phi(x)| .
$$

This means that $D u$ defines a linear form on the space of continuous vector fields, and by Riesz' representation Theorem it follows that it defines a Radon measure (precisely, a vector-valued (or signed) Borel measure on $\Omega$ which is finite on compact sets), which is globally bounded, and its norm (or variation $|D u|(\Omega)=\int_{\Omega}|D u|$ ) is precisely the total variation $J(u)$.

See for instance $[74,34,7]$ for details.

\subsubsection{Main properties of the total variation}

Lower semi-continuity The definition 1.1 has a few advantages. It can be introduced for any locally integrable function (without requiring any regularity or derivability). But also, $J(u)$ is written as a sup of linear forms

$$
L_{\phi}: u \mapsto-\int_{\Omega} u(x) \operatorname{div} \phi(x) d x
$$


which are continuous with respect to very weak topologies (in fact, with respect to the "distributional convergence" related to the space $\mathcal{D}^{\prime}$ introduced in the previous section).

For instance, if $u_{n} \rightarrow u$ in $L^{p}(\Omega)$ for any $p \in[1,+\infty$ ) (or weakly-* for $p=\infty$ ), or even in $L^{p}\left(\Omega^{\prime}\right)$ for any $\Omega^{\prime} \subset \subset \Omega$, then $L_{\phi} u_{n} \rightarrow L_{\phi} u$. But it follows that

$$
L_{\phi} u=\lim _{n} L_{\phi} u_{n} \leq \liminf _{n} J\left(u_{n}\right)
$$

and taking then the sup over all smooth fields $\phi$ with $|\phi(x)| \leq 1$ everywhere, we deduce that

$$
J(u) \leq \liminf _{n \rightarrow \infty} J\left(u_{n}\right),
$$

that is, $J$ is (sequentially) lower semi-continuous (l.s.c.) with respect to all the above mentioned topologies. [The idea is that a sup of continuous functions is l.s.c.]

In particular, it becomes obvious to show that with $F=J$, problem $(M A P c)$ has a solution. Indeed, consider a minimizing sequence for

$$
\min _{u} \mathcal{E}(u):=J(u)+\|u-g\|_{L^{2}(\Omega)}^{2},
$$

which is a sequence $\left(u_{n}\right)_{n \geq 1}$ such that $\mathcal{E}\left(u_{n}\right) \rightarrow \inf _{u} \mathcal{E}(u)$.

As $\mathcal{E}\left(u_{n}\right) \leq \mathcal{E}(0)<+\infty$ for $n$ large enough (we assume $g \in L^{2}(\Omega)$ ), and $J \geq 0$ ), we see that $\left(u_{n}\right)$ is bounded in $L^{2}(\Omega)$ and it follows that up to a subsequence (still denoted $\left(u_{n}\right)$, it converges weakly to some $u$, that is, for any $v \in L^{2}(\Omega)$,

$$
\int_{\Omega} u_{n}(x) v(x) d x \rightarrow \int_{\Omega} u(x) v(x) d x .
$$

But then it is known that

$$
\|u-g\|_{L^{2}} \leq \liminf _{n}\left\|u_{n}-g\right\|_{L^{2}},
$$

and since we also have (2), we deduce that

$$
\mathcal{E}(u) \leq \liminf _{n} \mathcal{E}\left(u_{n}\right)=\inf \mathcal{E}
$$

so that $u$ is a minimizer. 
Convexity Now, is $u$ unique? The second fundamental property of $J$ which we deduce from Definition 1.1 is its convexity: for any $u_{1}, u_{2}$ and $t \in[0,1]$,

$$
J\left(t u_{1}+(1-t) u_{2}\right) \leq t J\left(u_{1}\right)+(1-t) J\left(u_{2}\right) .
$$

It follows, again, because $J$ is the supremum of the linear (hence convex) functions $L_{\phi}$ : indeed, one clearly has

$$
L_{\phi}\left(t u_{1}+(1-t) u_{2}\right)=t L_{\phi}\left(u_{1}\right)+(1-t) L_{\phi}\left(u_{2}\right) \leq t J\left(u_{1}\right)+(1-t) J\left(u_{2}\right)
$$

and taking the sup in the left-hand side yields (3).

Hence in particular, if $u$ and $u^{\prime}$ are two solutions of (MAPc), then

$$
\begin{aligned}
\mathcal{E}\left(\frac{u+u^{\prime}}{2}\right) \leq \frac{\lambda}{2}\left(J(u)+J\left(u^{\prime}\right)\right)+\int_{\Omega} \mid & \frac{u+u^{\prime}}{2}-\left.g\right|^{2} d x \\
& =\frac{1}{2}\left(\mathcal{E}(u)+\mathcal{E}\left(u^{\prime}\right)\right)-\frac{1}{4} \int_{\Omega}\left(u-u^{\prime}\right)^{2} d x
\end{aligned}
$$

which would be strictly less than the $\inf$ of $\mathcal{E}$, unless $u=u^{\prime}$ : hence the minimizer of $(M A P c)$ exists, and is unique.

Homogeneity It is obvious for the definition that for each $u$ and $t>0$,

$$
J(t u)=t J(u)
$$

that is, $J$ is positively one-homogeneous.

\subsubsection{Functions with bounded variation}

We introduce the following definition:

Definition 1.2. The space $B V(\Omega)$ of functions with bounded variation is the set of functions $u \in L^{1}(\Omega)$ such that $J(u)<+\infty$, endowed with the norm $\|u\|_{B V(\Omega)}=$ $\|u\|_{L^{1}(\Omega)}+J(u)$.

This space is easily shown to be a Banach space. It is a natural (weak) "closure" of $W^{1,1}(\Omega)$. Let us state a few essential properties of this space. 
Meyers-Serrin's approximation Theorem We first state a theorem which shows that $B V$ function may be "well" approximated with smooth functions. This is a refinement of a classical theorem of Meyers and Serrin [53] for Sobolev spaces.

Theorem 1. Let $\Omega \subset \mathbb{R}^{N}$ be an open set and let $u \in B V(\Omega)$ : then there exists a sequence $\left(u_{n}\right)_{n \geq 1}$ of functions in $C^{\infty}(\Omega) \cap W^{1,1}(\Omega)$ such that

(i.) $u_{n} \rightarrow u$ in $L^{1}(\Omega)$,

(ii.) $J\left(u_{n}\right)=\int_{\Omega}\left|\nabla u_{n}(x)\right| d x \rightarrow J(u)=\int_{\Omega}|D u|$ as $n \rightarrow \infty$.

Before sketching the proof, let us recall that in Sobolev's spaces $W^{1, p}(\Omega), p<$ $\infty$, the thesis of this classical theorem is stronger, since one proves that $\| \nabla u_{n}-$ $\nabla u \|_{L^{p}} \rightarrow 0$, while here one cannot expect $J\left(u_{n}-u\right)=\int_{\Omega}\left|D u_{n}-D u\right| \rightarrow 0$ as $n \rightarrow \infty$. This is easily illustrated by the following example: let $\Omega=(-1,1)$, and $u(t)=-1$ if $t<0, u(t)=1$ if $t \geq 0$. Then, the sequence $u_{n}(t)=\tanh (n \times t)$ clearly converges to $u$, with

$$
\int_{-1}^{1} u_{n}^{\prime}(t) d t=2 \tanh (n) \rightarrow 2=J(u)
$$

as $n \rightarrow \infty$, but clearly $J\left(u_{n}-u\right) \approx 4$ for large $n$. In fact, it is clear that if $v$ is any smooth approximation of $u$ such as shown on Fig. 3 , then clearly the variation $J(u-v)$ of $w=u-v$ is given by

$$
\begin{aligned}
& \left|w\left(0^{-}\right)-w(-1)\right|+\left|w\left(0^{+}\right)-w\left(0^{-}\right)\right|+\left|w(1)-w\left(0^{+}\right)\right|= \\
& |v(0)-v(-1)|+2+|v(1)-v(0)| \approx 4
\end{aligned}
$$

and cannot be made arbitrarily small.

Proof. Let us now explain how Theorem 1 is proven. The idea is to smooth $u$ with a "mollifier" (or a "smoothing kernel"): as usual one considers a function $\eta \in C_{c}^{\infty}(B(0,1))$ with $\eta \geq 0$ and $\int_{B(0,1)} \eta(x) d x=1$. For each $\varepsilon>0$, one considers $\eta_{\varepsilon}(x):=(1 / \varepsilon)^{N} \eta(x / \varepsilon)$ : then, $\eta_{\varepsilon}$ has support in the ball $B(0, \varepsilon)$, and $\int_{\mathbb{R}^{N}} \eta_{\varepsilon} d x=1$. If $u \in L^{1}\left(\mathbb{R}^{N}\right)$, it is then classical that the functions

$$
u_{\varepsilon}(x)=u * \eta_{\varepsilon}(x):=\int_{\mathbb{R}^{N}} u(y) \eta_{\varepsilon}(x-y) d y=\int_{B(0, \varepsilon)} u(x-y) \eta_{\varepsilon}(y) d y
$$

are smooth (because the first expression of the convolution product can be derived infinitely many times under the integral), and converge to $u$, in $L^{1}\left(\mathbb{R}^{N}\right)$, as $\varepsilon \rightarrow 0$ 


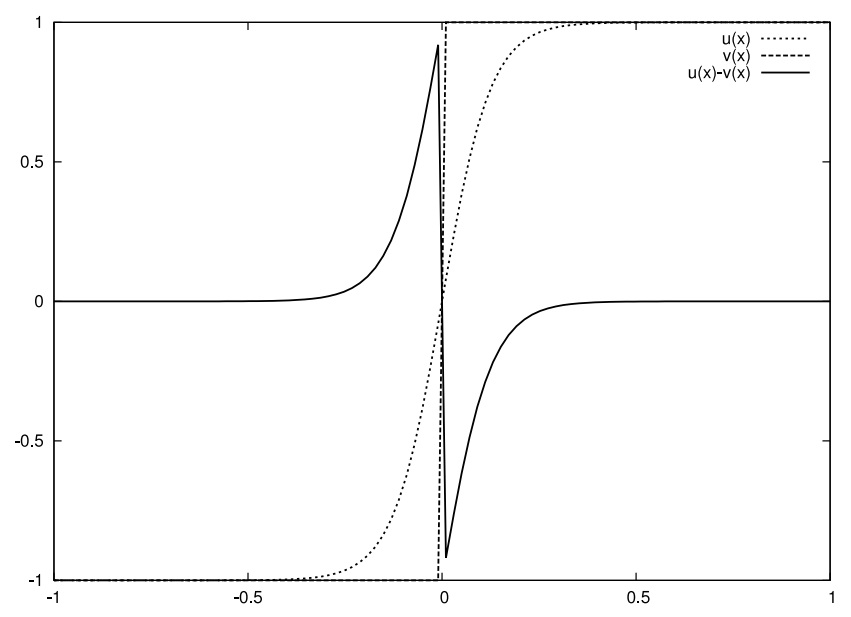

Figure 3: Smooth approximation of a step function

(the convergence is first easily shown for continuous function with compact support, and follows by density for $L^{1}$ functions).

Then, if $u \in B V\left(\mathbb{R}^{N}\right)$, one also have that for any $\phi \in C_{c}^{\infty}\left(\mathbb{R}^{N} ; \mathbb{R}^{N}\right)$ with $|\phi| \leq 1$ a.e., (to simplify we assume $\eta$ is even)

$$
\begin{aligned}
& \int_{\mathbb{R}^{N}} \phi(x) \cdot \nabla u_{\varepsilon}(x) d x \\
&=\int_{\mathbb{R}^{N}} u_{\varepsilon}(x) \operatorname{div} \phi(x) d x=\int_{\mathbb{R}^{N}} \int_{\mathbb{R}^{N}} \eta_{\varepsilon}(x-y) u(y) \operatorname{div} \phi(x) d y d x \\
&=\int_{\mathbb{R}^{N}} u(y) \operatorname{div}\left(\phi_{\varepsilon}\right)(y) d y
\end{aligned}
$$

where we have used Fubini's theorem, and the fact that $(\operatorname{div} \phi)_{\varepsilon}=\operatorname{div}\left(\phi_{\varepsilon}\right)$. By Definition 1.1, this is less than $J(u)$. Taking then the sup on all admissible $\phi$ 's, we end up with

$$
J\left(u_{\varepsilon}\right)=\int_{\mathbb{R}^{N}}\left|\nabla u_{\varepsilon}\right| d x \leq J(u)
$$

for all $\varepsilon>0$. Combined with (2), it follows that

$$
\lim _{\varepsilon \rightarrow 0} J\left(u_{\varepsilon}\right)=J(u) .
$$

This shows the Theorem, when $\Omega=\mathbb{R}^{N}$.

When $\Omega \neq \mathbb{R}^{N}$, this theorem is shown by a subtle variant of the classical proof of Meyers-Serrin's theorem [53], see for instance [40] or [7, Thm. 3.9] for details. Let us insist that the result is not straightforward, and, in particular, that in general the function $u_{n}$ can not be supposed to be smooth up to the boundary. 
Rellich's compactness theorem The second important property of $B V$ functions is the following compactness theorem:

Theorem 2. Let $\Omega \subset \mathbb{R}^{N}$ be a bounded domain with Lipschitz boundary, and let $\left(u_{n}\right)_{n \geq 1}$ be a sequence of functions in $B V(\Omega)$ such that $\sup _{n}\left\|u_{n}\right\|_{B V}<+\infty$. Then there exists $u \in B V(\Omega)$ and a subsequence $\left(u_{n_{k}}\right)_{k \geq 1}$ such that $u_{n_{k}} \rightarrow u$ (strongly) in $L^{1}(\Omega)$ as $k \rightarrow \infty$.

Proof. If we assume that the theorem is know for functions in $W^{1,1}(\Omega)$, then the extension to $B V$ functions simply follows from Thm 1 . Indeed, for each $n$, we can find $u_{n}^{\prime} \in C^{\infty}(\Omega) \cap W^{1,1}(\Omega)$ with $\left\|u_{n}-u_{n}^{\prime}\right\|_{L^{1}} \leq 1 / n$ and $\left\|u_{n}^{\prime}\right\|_{B V(\Omega)} \leq\left\|u_{n}\right\|_{B V(\Omega)}+$ $1 / n$. Then, we apply Rellich's compactness theorem in $W^{1,1}(\Omega)$ to the sequence $u_{n}^{\prime}$ : it follows that there exists $u \in L^{1}(\Omega)$ and a subsequence $\left(u_{n_{k}}^{\prime}\right)_{k}$ with $u_{n_{k}}^{\prime} \rightarrow u$ as $k \rightarrow \infty$. Clearly, we have $\left\|u_{n_{k}}-u\right\|_{L^{1}} \leq 1 / n_{k}+\left\|u_{n_{k}}^{\prime}-u\right\|_{L^{1}} \rightarrow 0$ as $k \rightarrow \infty$. Moreover, $u \in B V(\Omega)$, since its variation is bounded as follows from (2).

A complete proof (including the proof of Rellich's Thm) is found in [7], proof of Thm 3.23. The regularity of the domain $\Omega$ is crucial here, since the proof relies on an extension argument outside of $\Omega$ : it needs the existence of a linear "extension" operator $T: B V(\Omega) \rightarrow B V\left(\Omega^{\prime}\right)$ for any $\Omega^{\prime} \supset \supset$, such that for each $u \in B V(\Omega), T u$ has compact support in $\Omega^{\prime}, T u(x)=u(x)$ for a.e. $x \in \Omega$, and $\|T u\|_{B V\left(\Omega^{\prime}\right)} \leq C\|u\|_{B V(\Omega)}$. Then, the proof follows by mollifying the sequence $T u_{n}$, introducing the smooth functions $\eta_{\varepsilon} * T u_{n}$, applying Ascoli-Arzelà's theorem to the mollified functions, and a diagonal argument.

Sobolev's inequalities We observe here that the classical inequalities of Sobolev:

$$
\|u\|_{L^{\frac{N}{N-1}}\left(\mathbb{R}^{N}\right)} \leq C \int_{\mathbb{R}^{N}}|D u|
$$

if $u \in L^{1}\left(\mathbb{R}^{N}\right)$, and Poincaré-Sobolev:

$$
\|u-m\|_{L^{\frac{N}{N-1}}(\Omega)} \leq C \int_{\mathbb{R}^{N}}|D u|
$$

where $\Omega$ is bounded with Lipschitz boundary, and $m$ is the average of $u$ on $\Omega$, valid for $W^{1,1}$ functions, clearly also hold for $B V$ function as can be deduced from Thm 1. 


\subsection{The perimeter. Sets with finite perimeter}

\subsubsection{Definition, and an inequality}

Definition 1.3. A measurable set $E \subset \Omega$ is a set of finite perimeter in $\Omega$ (or Caccioppoli set) if and only if $\chi_{E} \in B V(\Omega)$. The total variation $J\left(\chi_{E}\right)$ is the perimeter of $E$ in $\Omega$, denoted by $\operatorname{Per}(E ; \Omega)$. If $\Omega=\mathbb{R}^{N}$, we simply denote $\operatorname{Per}(E)$.

We observe that a "set" here is understood as a measurable set in $\mathbb{R}^{N}$, and that this definition of the perimeter makes it depend on $E$ only up to sets of zero Lebesgue measure. In general, in what follows, the sets we will considers will be rather equivalence classes of sets which are equal up to Lebesgue negligible sets.

The following inequality is an essential property of the perimeter: for any $A, B \subseteq$ $\Omega$ sets of finite perimeter, we have

$$
\operatorname{Per}(A \cup B ; \Omega)+\operatorname{Per}(A \cap B ; \Omega) \leq \operatorname{Per}(A ; \Omega)+\operatorname{Per}(B ; \Omega) .
$$

Proof. The proof is as follows: we can consider, invoking Thm 1, two sequences $u_{n}, v_{n}$ of smooth functions, such that $u_{n} \rightarrow \chi_{A}, v_{n} \rightarrow \chi_{B}$, and

$$
\int_{\Omega}\left|\nabla u_{n}(x)\right| d x \rightarrow \operatorname{Per}(A ; \Omega) \text { and } \int_{\Omega}\left|\nabla v_{n}(x)\right| d x \rightarrow \operatorname{Per}(B ; \Omega)
$$

as $n \rightarrow \infty$. Then, it is easy to check that $u_{n} \vee v_{n}=\max \left\{u_{n}, v_{n}\right\} \rightarrow \chi_{A \cup B}$ as $n \rightarrow \infty$, while $u_{n} \wedge v_{n}=\min \left\{u_{n}, v_{n}\right\} \rightarrow \chi_{A \cap B}$ as $n \rightarrow \infty$. We deduce, using (2), that

$$
\operatorname{Per}(A \cup B ; \Omega)+\operatorname{Per}(A \cap B ; \Omega) \leq \liminf _{n \rightarrow \infty} \int_{\Omega}\left|\nabla\left(u_{n} \vee v_{n}\right)\right|+\left|\nabla\left(u_{n} \wedge v_{n}\right)\right| d x .
$$

But for almost all $x \in \Omega,\left|\nabla\left(u_{n} \vee v_{n}\right)(x)\right|+\left|\nabla\left(u_{n} \wedge v_{n}\right)(x)\right|=\left|\nabla u_{n}(x)\right|+\left|\nabla v_{n}(x)\right|$, so that (7) follows from (9) and (8).

\subsubsection{The reduced boundary, and a generalization of Green's formula}

It is shown that if $E$ is a set of finite perimeter in $\Omega$, then the derivative $D \chi_{E}$ can be expressed as

$$
D \chi_{E}=\nu_{E}(x) \mathcal{H}^{N-1}\left\llcorner\partial^{*} E\right.
$$

where $\nu_{E}(x)$ and $\partial^{*} E$ can be defined as follows: $\partial^{*} E$ is the set of points $x$ where the "blow-up" sets

$$
E_{\varepsilon}=\{y \in B(0,1): x+\varepsilon y \in E\}
$$


converge as $\varepsilon$ to 0 to a semi-space $P_{\nu_{E}(x)}=\left\{y: y \cdot \nu_{E}(x) \geq 0\right\} \cap B(0,1)$ in $L^{1}(B(0,1))$, in the sense that their characteristic functions converge, or in other words

$$
\left|E_{\varepsilon} \backslash P_{\nu_{E}(x)}\right|+\left|P_{\nu_{E}(x)} \backslash E_{\varepsilon}\right| \rightarrow 0
$$

as $\varepsilon \rightarrow 0$. This defines also the (inner) normal vector $\nu_{E}(x)$.

The set $\partial^{*} E$ is called the "reduced" boundary of $E$ (the "true" definition of the reduced boundary is a bit more precise and the precise set slightly smaller than ours, but still (10) is true with our definition, see [7, Chap. 3]).

Eq. (10) means that for any $C^{1}$ vector field $\phi$, one has

$$
\int_{E} \operatorname{div} \phi(x) d x=-\int_{\partial^{*} E} \phi \cdot \nu_{E}(x) d \mathcal{H}^{N-1}(x)
$$

which is a sort of generalization of Green's formula to sets of finite perimeter.

This generalization is useful as shows the following example: let $x_{n} \in(0,1)^{2}, n \geq$ 1 , be the sequence of rational points (in $\left.\mathbb{Q}^{2} \cap(0,1)^{2}\right)$, and let $E=\bigcup_{n \geq 1} B\left(x_{n}, \varepsilon 2^{-n}\right.$ ), for some $\varepsilon>0$ fixed.

Then, one sees that $E$ is an open, dense set in $(0,1)^{2}$. In particular its "classical" (topological) boundary $\partial E$ is very big, it is $[0,1]^{2} \backslash E$ and has Lebesgue measure equal to $1-|E| \geq 1-\pi \varepsilon^{2} / 3$. In particular its length is infinite.

However, one can show that $E$ is a finite perimeter set, with perimeter less than $\sum_{n} 2 \pi \varepsilon 2^{-n}=\pi \varepsilon$. Its "reduced boundary" is, up to the intersections (which are negligible), the set

$$
\partial^{*} E \approx \bigcup_{n \geq 1} \partial B\left(x_{n}, \varepsilon 2^{-n}\right) .
$$

One shows that this "reduced boundary" is always, as in this simple example, a rectifiable set, that is, a set which can be almost entirely covered with a countable union of $C^{1}$ hypersurfaces, up to a set of Hausdorff $\mathcal{H}^{N-1}$ measure zero: there exist $\left(\Gamma_{i}\right)_{i \geq 1}$, hypersurfaces of regularity $C^{1}$, such that

$$
\partial^{*} E \subset \mathcal{N} \cup\left(\bigcup_{i=1}^{\infty} \Gamma_{i}\right), \mathcal{H}^{N-1}(\mathcal{N})=0 .
$$

In particular, $\mathcal{H}^{N-1}$-a.e., the normal $\nu_{E}(x)$ is a normal to the surface $(\mathrm{s}) \Gamma_{i}$ such that $x \in \Gamma_{i}$. 


\subsubsection{The isoperimetric inequality}

For $u=\chi_{E}$, equation (5) becomes the celebrated isoperimetric inequality:

$$
|E|^{\frac{N-1}{N}} \leq C \operatorname{Per}(E)
$$

for all finite-perimeter set $E$ of bounded volume, with the best constant $C$ reached by balls:

$$
C^{-1}=N\left(\omega_{N}\right)^{1 / N}
$$

where $\omega_{N}=|B(0,1)|$ is the volume of the unit ball in $\mathbb{R}^{N}$.

\subsection{The co-area formula}

We now can state a fundamental property of $B V$ functions, which will be the key of our analysis in the next sections dealing with applications. This is the famous "co-area" formula of Federer and Fleming:

Theorem 3. Let $u \in B V(\Omega)$ : then for a.e. $s \in \mathbb{R}$, the set $\{u>s\}$ is a finiteperimeter set in $\Omega$, and one has

$$
J(u)=\int_{\Omega}|D u|=\int_{-\infty}^{+\infty} \operatorname{Per}(\{u>s\} ; \Omega) d s .
$$

It means that the total variation of a function is also the accumulated surfaces of all its level sets. The proof of this result is quite complicated (we refer to [36, $34,74,7])$ but let us observe that:

- It is relatively simple if $u=p \cdot x$ is an affine function, defined for instance on a simplex $T$ (or in fact any open set). Indeed, in this case, $J(u)=|T||p|$, and $\partial\{u>s\}$ are hypersurfaces $\{p \cdot x=s\}$, and it is not too difficult to compute the integral $\int_{s} \mathcal{H}^{N-1}(\{p \cdot x=s\})$;

- For a general $u \in B V(\Omega)$, we can approximate $u$ with piecewise affine functions $u_{n}$ with $\int_{\Omega}\left|\nabla u_{n}\right| d x \rightarrow J(u)$. Indeed, one can first approximate $u$ with the smooth functions provided by Thm 1 , and then these smooth functions by piecewise affine functions using the standard finite elements theory. Then, we will obtain using (2) and Fatou's lemma that $\int_{\mathbb{R}} \operatorname{Per}(\{u>s\} ; \Omega) d s \leq J(u)$;

- The reverse inequality $J(u) \leq \int_{\mathbb{R}} \operatorname{Per}(\{u>s\} ; \Omega) d s=\int_{\mathbb{R}} J\left(\chi_{\{u>s\}}\right) d s$, can easily be deduced by noticing that if $\phi \in C_{c}^{\infty}(\Omega)$ with $\|\phi\| \leq 1$, one has 
$\int_{\Omega} \operatorname{div} \phi d x=0$, so that (using Fubini's theorem)

$$
\begin{aligned}
& \int_{\Omega} u \operatorname{div} \phi d x= \\
& \int_{\{u>0\}} \int_{0}^{u(x)} d s \operatorname{div} \phi(x) d x-\int_{\{u<0\}} \int_{u(x)}^{0} d s \operatorname{div} \phi(x) d x= \\
& \int_{0}^{\infty} \int_{\Omega} \chi_{\{u>s\}}(x) \operatorname{div} \phi(x) d x d s-\int_{-\infty}^{0} \int_{\Omega}\left(1-\chi_{\{u>s\}}(x)\right) \operatorname{div} \phi(x) d x d s \\
& =\int_{-\infty}^{\infty} \int_{\{u>s\}} \operatorname{div} \phi d x d s \leq \int_{-\infty}^{\infty} \operatorname{Per}(\{u>s\} ; \Omega) d s
\end{aligned}
$$

and taking then the sup over all admissible $\phi$ 's in the leftmost term.

Remark: observe that (7) also follows easily from $(C A)$, indeed, let $u=\chi_{A}+\chi_{B}$, then $J(u) \leq J\left(\chi_{A}\right)+J\left(\chi_{B}\right)=\operatorname{Per}(A ; \Omega)+\operatorname{Per}(B ; \Omega)$, while from $(C A)$ we get that

$$
J(u)=\int_{0}^{2} \operatorname{Per}\left(\left\{\chi_{A}+\chi_{B}>s\right\} ; \Omega\right) d s=\operatorname{Per}(A \cup B ; \Omega)+\operatorname{Per}(A \cap B ; \Omega) .
$$

\subsection{The derivative of a $B V$ function (*)}

To end up this theoretical section on $B V$ functions, we mention an essential result on the measure $D u$, defined for any $u \in B V(\Omega)$ by

$$
\int \phi(x) \cdot D u(x)=-\int u(x) \operatorname{div} \phi(x) d x
$$

for any smooth enough vector field $\phi$ with compact support. As mentioned in Section 1.2.2, it is a bounded Radon measure. A derivation theorem due to Radon and Nikodym (and a refined version due to Besicovitch) shows that such a measure can be decomposed with respect to any positive radon measure $\mu$ into

$$
D u=f(x) d \mu+\nu
$$

where $\mu$-a.e.,

$$
f(x)=\lim _{\rho \rightarrow 0} \frac{D u(B(x, \rho))}{\mu(B(x, \rho)}
$$

(and in particular the theorem states that the limit exists a.e.), $f \in L_{\mu}^{1}(\Omega)$, that is, $\int_{\Omega}|f| d \mu<+\infty$, and $\nu \perp \mu$, which means that there exists a Borel set $E \subset \Omega$ such that $|\nu|(\Omega \backslash E)=0, \mu(E)=0$.

If the function $u \in W^{1,1}(\Omega)$, then $D u=\nabla u(x) d x$, with $\nabla u$ the "weak gradient" a vector-valued function in $L^{1}\left(\Omega ; \mathbb{R}^{N}\right)$. Hence, the decomposition (14) with $\mu=d x$ 
(the Lebesgue measure), holds with $f=\nabla u$ and $\nu=0$, and one says that $D u$ is "absolutely continuous" with respect to Lebesgue's measure. This is not true anymore for a generic function $u \in B V(\Omega)$. One has

$$
D u=\nabla u(x) d x+D^{s} u
$$

where the "singular part" $D^{s} u$ vanishes if and only if $u \in W^{1,1}$, and $\nabla u \in L^{1}\left(\Omega ; \mathbb{R}^{N}\right)$ is the "approximate gradient" of $u$.

The singular part can be further decomposed. Let us call $J_{u}$ the "jump set" of $u$, defined as follows:

Definition 1.4. Given $u \in B V(\Omega)$, we say that $x \in J_{u}$ if and only if there exist $u_{-}(x), u_{+}(x) \in \mathbb{R}$ with $u_{-}(x) \neq u_{+}(x)$, and $\nu_{u}(x) \in \mathbb{R}^{N}$ a unit vector such that the functions, defined for $y \in B(0,1)$ for $\varepsilon>0$ small enough

$$
y \mapsto u(x+\varepsilon y)
$$

converge as $\varepsilon \rightarrow 0$, in $L^{1}(B(0,1))$, to the function

$$
y \mapsto u_{-}(x)+\left(u_{+}(x)-u_{-}(x)\right) \chi_{\left\{y \cdot \nu_{u}(x) \geq 0\right\}}
$$

which takes value $u_{+}(x)$ in the half-space $\left\{y \cdot \nu_{u}(x) \geq 0\right\}$, and $u_{-}(x)$ in the other half-space $\left\{y \cdot \nu_{u}(x)<0\right\}$

In particular, this is consistent with our definition of $\partial^{*} E$ in Section 1.3: $\partial^{*} E=$ $J_{\chi_{E}}$, with $\left(\chi_{E}\right)_{+}(x)=1,\left(\chi_{E}\right)_{-}(x)=0$, and $\nu_{\chi_{E}}(x)=\nu_{E}$. The triple $\left(u_{-}, u_{+}, \nu_{u}\right)$ is almost unique: it is unique up to the permutation $\left(u_{+}, u_{-},-\nu_{u}\right)$. For a scalar function $u$, the canonical choice is to take $u_{+}>u_{-}$, whereas for vectorial $B V$ functions, one must fix some arbitrary rule.

One can show that $J_{u}$ is a rectifiable set (see Section 1.3, eq. (12)), in fact, it is a countable union of rectifiable sets since one can always write

$$
J_{u} \subseteq \bigcup_{n \neq m} \partial^{*}\left\{u>s_{n}\right\} \cap \partial^{*}\left\{u>s_{m}\right\}
$$

for some countable, dense sequence $\left(s_{n}\right)_{n \geq 1}$ : the jump set is where two different level sets meet.

One then has the following fundamental result: 
Theorem 4 (Federer-Volpert). Let $u \in B V(\Omega)$ : then one has

$$
D u=\nabla u(x) d x+C u+\left(u_{+}(x)-u_{-}(x)\right) \nu_{u}(x) d \mathcal{H}^{N-1}\left\llcorner J_{u}\right.
$$

where Cu is the "Cantor part" of Du, which is singular with respect to the Lebesgue measure, and vanishes on any set $E$ with $\mathcal{H}^{N-1}(E)<+\infty$. In other words, for any $\phi \in C_{c}^{1}\left(\Omega ; \mathbb{R}^{N}\right)$,

$$
\begin{aligned}
-\int_{\Omega} u(x) \operatorname{div} \phi(x) & d x=\int_{\Omega} \nabla u(x) \cdot \phi(x) d x \\
+ & \int_{\Omega} \phi(x) \cdot C u(x)+\int_{J_{u}}\left(u_{+}(x)-u_{-}(x)\right) \phi(x) \cdot \nu_{u}(x) d x .
\end{aligned}
$$

Observe that (15) is a generalized version of (11).

As we have seen, an example of a function with absolutely continuous derivative is given by any function $u \in W^{1,1}(\Omega)$ (or more obviously $u \in C^{1}(\bar{\Omega})$ ).

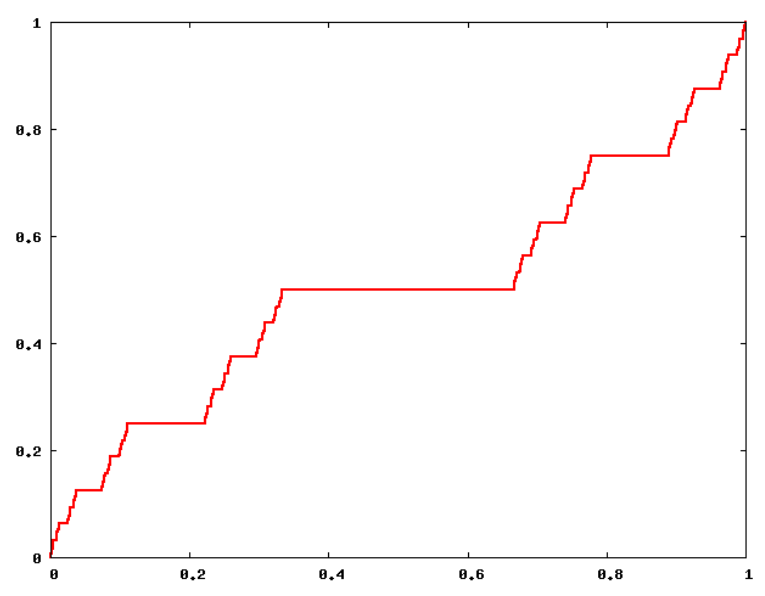

Figure 4: The "devil's staircase" or Cantor-Vitali function

An example of a function with derivative a pure jump is given by $u=\chi_{E}, E$ a Caccioppoli set (see Section 1.3). A famous example of a function with derivative purely Cantorian is the Cantor-Vitali function, obtained as follows: $\Omega=(0,1)$ and we let $u_{0}(t)=t$, and for any $n \geq 0$,

$$
u_{n+1}(t)= \begin{cases}\frac{1}{2} u_{n}(3 t) & 0 \leq t \leq \frac{1}{3} \\ \frac{1}{2} & \frac{1}{3} \leq t \leq \frac{2}{3} \\ \frac{1}{2}\left(u_{n}(3 t-2)+1\right) & \frac{2}{3} \leq t \leq 1\end{cases}
$$


Then, one checks that

$$
\sup _{(0,1)}\left|u_{n+1}-u_{n}\right|=\frac{1}{2} \sup _{(0,1)}\left|u_{n}-u_{n-1}\right|=\frac{1}{2^{n}} \times \frac{1}{6}
$$

so that $\left(u_{n}\right)_{n \geq 1}$ is a Cauchy sequence and converges uniformly to some function $u$. This function (see Fig. 4) is constant on each interval the complement of the triadic Cantor set, which has zero measure in $(0,1)$. Hence, almost everywhere, its classical derivative exists and is zero. One can deduce that the derivative $D u$ is singular with respect to Lebesgue's measure. On the other hand, it is continuous as a uniform limit of continuous functions, hence $D u$ has no jump part. In fact, $D u=C u$, which, in this case, is the measure $\mathcal{H}^{\ln 2 / \ln 3}\left\llcorner C / \mathcal{H}^{\ln 2 / \ln 3}(C)\right.$.

\section{Some functionals where the total variation appears}

\subsection{Perimeter minimization}

In quite a few applications it is important to be able to solve the following problem:

$$
\min _{E \subset \Omega} \lambda \operatorname{Per}(E ; \Omega)-\int_{E} g(x) d x
$$

The intuitive idea is as follows: if $\lambda=0$, then this will simply choose $E=\{g \geq$ $0\}$ : that is, we find the set $E$ by thresholding the values of $g$ at 0 . Now, imagine that this is precisely what we would like to do, but that $g$ has some noise, so that a brutal thresholding of its value will produce a very irregular set. Then, choosing $\lambda>0$ in (16) will start regularizing the set $\{g>0\}$, and the high values of $\lambda$ will produce a very smooth, but possibly quite approximate, version of that set.

We now state a proposition which is straighforward for people familiar with linear programming and "LP"-relaxation:

Proposition 2.1. Problem (16) is convex. In fact, it can be relaxed as follows:

$$
\min _{u \in B V(\Omega ;[0,1])} \lambda J(u)-\int_{\Omega} u(x) g(x) d x
$$

and given any solution $u$ of the convex problem (17), and any value $s \in[0,1)$, the set $\{u>s\}$ (or $\{u \geq s\}$ for $s \in(0,1])$ is a solution of (16). 
Proof. This is a consequence of the co-area formula. Denote by $m^{*}$ the minimum value of Problem (16). One has

$$
\begin{aligned}
\int_{\Omega} u(x) g(x) d x=\int_{\Omega} & \left(\int_{0}^{u(x)} d s\right) g(x) d x \\
= & \int_{\Omega} \int_{0}^{1} \chi_{\{u>s\}}(x) g(x) d s d x=\int_{0}^{1} \int_{\{u>s\}} g(x) d x d s
\end{aligned}
$$

so that, if we denote $\mathcal{E}(E)=\lambda \operatorname{Per}(E ; \Omega)-\int_{E} g(x) d x$, it follows from (18) and $(C A)$ that for any $u \in B V(\Omega)$ with $0 \leq u \leq 1$ a.e.,

$$
\lambda J(u)-\int_{\Omega} u(x) g(x) d x=\int_{0}^{1} \mathcal{E}(\{u>s\}) d s \geq m^{*} .
$$

Hence the minimum value of (17) is larger than $m^{*}$, on the other hand, it is also less since (16) is just (17) restricted to characteristic functions. Hence both problems have the same values, and it follows from (19) that if $\lambda J(u)-\int_{\Omega} u g d x=m^{*}$, that is, if $u$ is a minimizer for (17), then for a.e. $s \in(0,1),\{u>s\}$ is a solution to (16). Denote by $S$ the set of such values of $s$. Now let $s \in[0,1)$, and let $\left(s_{n}\right)_{n \geq 1}$ be a decreasing sequence of values such that $s_{n} \in S$ and $s_{n} \rightarrow s$ as $n \rightarrow \infty$. Then, $\{u>s\}=\bigcup_{n \geq 1}\left\{u>s_{n}\right\}$, and, in fact, $\lim _{n \rightarrow \infty}\left\{u>s_{n}\right\}=\{u>s\}$ (the limit is in the $L^{1}$ sense, that is, $\int_{\Omega}\left|\chi_{\left\{u>s_{n}\right\}}-\chi_{\{u>s\}}\right| d x \rightarrow 0$ as $n \rightarrow \infty$. Using (2), it follows

$$
m^{*} \leq \mathcal{E}(\{u>s\}) \leq \liminf _{n \rightarrow \infty} \mathcal{E}\left(\left\{u>s_{n}\right\}\right)=m^{*}
$$

so that $s \in S:$ it follows that $S=[0,1)$.

The meaning of this result is that it is always possible to solve a problem such as (16) despite it apparently looks non-convex, and despite the fact the solution might be nonunique (although we will soon see that it is quite "often" unique). This has been observed several times in the past [26], and probably the first time for numerical purposes in [14]. In Section 3 we will address the issues of algorithms to tackle this kind of problems.

\subsection{The Rudin-Osher-Fatemi problem}

We now concentrate on problem $(M A P c)$ with $F(u)=\lambda J(u)$ as a regularizer, that is, on the celebrated "Rudin-Osher-Fatemi" problem (in the "pure denoising case": we will not consider any operator $A$ as in $(M A P))$ :

$$
\min _{u} \lambda J(u)+\frac{1}{2} \int_{\Omega}|u(x)-g(x)|^{2} d x
$$


As mentioned in section 1.2.3, this problem has a unique solution (it is strictly convex).

Let us now show that as in the previous section, the level sets $E_{s}=\{u>s\}$ solve a particular variational problem (of the form (16), but with $g(x)$ replaced with some $s$-dependent function). This will be of particular interest for our further analysis.

\subsubsection{The Euler-Lagrange equation}

Formally:

$$
-\lambda \operatorname{div} \frac{D u}{|D u|}+u-g=0
$$

but this is hard to interpret. In particular because one can show there is always "staircasing", as soon as $g \in L^{\infty}(\Omega)$, so that there always are large areas where " $D u=0 "$.

On can interpret the equation in the viscosity sense. Or try to derive the "correct" Euler-Lagrange equation in the sense of convex analysis. This requires to define properly the "subgradient" of $J$.

Definition 2.2. For $X$ a Hilbert space, the subgradient of a convex function $F$ : $X \rightarrow(-\infty,+\infty]$ is the operator $\partial F$ which maps $x \in X$ to the (possibly empty set)

$$
\partial F(x)=\{p \in X: F(y) \geq F(x)+\langle v, y-x\rangle \forall y \in X\}
$$

We introduce the set

$$
\mathcal{K}=\left\{-\operatorname{div} \phi: \phi \in C_{c}^{\infty}\left(\Omega ; \mathbb{R}^{N}\right):|\phi(x)| \leq 1 \forall x \in \Omega\right\}
$$

and the closure $K$ if $\mathcal{K}$ in $L^{2}(\Omega)$, which is shown to be

$$
K=\left\{-\operatorname{div} z: z \in L^{\infty}\left(\Omega ; \mathbb{R}^{N}\right):-\operatorname{div}\left(z \chi_{\Omega}\right) \in L^{2}\left(\mathbb{R}^{N}\right)\right\}
$$

where the last condition means that

(i.) $-\operatorname{div} z \in L^{2}(\Omega)$, i.e., there exists $\gamma \in L^{2}(\Omega)$ such that $\int_{\Omega} \gamma u d x=\int_{\Omega} z \cdot \nabla u d x$ for all smooth $u$ with compact support;

(ii.) the above also holds for $u \in H^{1}(\Omega)$ (not compactly supported), in other words $z \cdot \nu_{\Omega}=0$ on $\partial \Omega$ in the weak sense. 
Definition 1.1 defines $J$ as

$$
J(u)=\sup _{p \in \mathcal{K}} \int_{\Omega} u(x) p(x) d x
$$

so that if $u \in L^{2}(\Omega)$ it is obvious that, also,

$$
J(u)=\sup _{p \in K} \int_{\Omega} u(x) p(x) d x .
$$

In fact, one shows that $K$ is the largest set in $L^{2}(\Omega)$ such that $(21)$ holds for any $u \in L^{2}(\Omega)$, in other words

$$
K=\left\{p \in L^{2}(\Omega): \int_{\Omega} p(x) u(x) d x \leq J(u) \forall u \in L^{2}(\Omega)\right\}
$$

Then, if we consider $J$ as a functional over the Hilbert space $X=L^{2}(\Omega)$, we have:

Proposition 2.3. For $u \in L^{2}(\Omega)$,

$$
\partial J(u)=\left\{p \in K: \int_{\Omega} p(x) u(x) d x=J(u)\right\}
$$

Proof. It is not hard to check that if $p \in K$ and $\int_{\Omega} p u d x=J(u)$, then $p \in \partial J(u)$, indeed, for any $v \in L^{2}(\Omega)$, using (21),

$$
J(v) \geq \int_{\Omega} p(x) v(x) d x=J(u)+\int_{\Omega}(v(x)-u(x)) p(x) d x .
$$

The converse inclusion can be proved as follows: if $p \in \partial J(u)$, then for any $t>0$ and $v \in \mathbb{R}^{N}$, as $J$ is one-homogeneous (4),

$$
t J(v)=J(t v) \geq J(u)+\int_{\Omega} p(x)(t v(x)-u(x)) d x,
$$

dividing by $t$ and sending $t \rightarrow \infty$, we get $J(v) \geq \int_{\Omega} p v d x$, hence $p \in K$, by (22). On the other hand, sending $t$ to 0 shows that $J(u) \leq \int_{\Omega} p u d x$ which shows our claim.

Remark We see that $K=\partial J(0)$. 
The Euler-Lagrange equation for $(R O F)$ We can now derive the equation satisfied by $u$ which minimizes $(R O F)$ : for any $v$ in $L^{2}(\Omega)$, we have

$$
\begin{array}{r}
\lambda J(v) \geq \lambda J(u)+\frac{1}{2} \int_{\Omega}(u-g)^{2}-(v-g)^{2} d x=\lambda J(u)+\int_{\Omega}(u-v)\left(\frac{u+v}{2}-g\right) d x \\
=\lambda J(u)+\int_{\Omega}(v-u)(g-u) d x-\frac{1}{2} \int_{\Omega}(u-v)^{2} d x .
\end{array}
$$

In particular, for any $t \in \mathbb{R}$,

$$
\lambda(J(u+t(v-u))-J(u))-t \int_{\Omega}(v-u)(g-u) d x \geq-\frac{t^{2}}{2} \int_{\Omega}(v-u)^{2} d x .
$$

The left-hand side of the last expression is a convex function of $t \in \mathbb{R}$, one can show quite easily that a convex function which is larger than a concave parabola and touches at the maximum point $(t=0)$ must be everywhere larger than the maximum of the parabola (here zero).

We deduce that

$$
\lambda(J(u+t(v-u))-J(u))-t \int_{\Omega}(v-u)(g-u) d x \geq 0
$$

for any $t$, in particular for $t=1$, which shows that $\frac{g-u}{\lambda} \in \partial J(u)$. Conversely, if this is true, then obviously (24) holds so that $u$ is the minimizer of $J$. It follows that the Euler-Lagrange equation for $(R O F)$ is

$$
\lambda \partial J(u)+u-g \ni 0
$$

which, in view of (23) and the characterization of $K$, is equivalent to the existence of $z \in L^{\infty}\left(\Omega ; \mathbb{R}^{N}\right)$ with:

$$
\begin{cases}-\lambda \operatorname{div} z(x)+u(x)=g(x) & \text { a.e. } x \in \Omega \\ |z(x)| \leq 1 & \text { a.e. } x \in \Omega \\ z \cdot \nu=0 & \text { on } \partial \Omega \text { (weakly) } \\ z \cdot D u=|D u|, & \end{cases}
$$

the last equation being another way to write that $\int(-\operatorname{div} z) u d x=J(u)$.

If $u$ were smooth and $\nabla u \neq 0$, the last condition ensures that $z=\nabla u /|\nabla u|$ and we recover (20).

In all cases, we see that $z$ must be orthogonal to the level sets of $u$ (from $|z| \leq 1$ and $z \cdot D u=|D u|)$, so that $-\operatorname{div} z$ is still the curvature of the level sets. 
In $1 \mathrm{D}, z$ is a scalar and the last condition is $z \cdot u^{\prime}=\left|u^{\prime}\right|$, so that $z \in\{-1,+1\}$ whenever $u$ is not constant, while $\operatorname{div} z=z^{\prime}$ : we see that the equation becomes $u=g$ or $u=$ constant (and in particular staircasing is a necessity if $g$ is not monotonous).

\subsubsection{The problem solved by the level sets}

We introduce the following problems, parameterized by $s \in \mathbb{R}$ :

$$
\min _{E} \lambda \operatorname{Per}(E ; \Omega)+\int_{E} s-g(x) d x .
$$

Given $s \in \mathbb{R}$, let us denote by $E_{s}$ a solution of $\left(R O F_{s}\right)$ (whose existence follows in a straightforward way from Rellich's theorem 2 and (2)).

Then the following holds

Lemma 2.4. Let $s^{\prime}>s$ : then $E_{s^{\prime}} \subseteq E_{s}$.

This lemma is found for instance in [4, Lem. 4]. Its proof is very easy.

Proof. We have (to simplify we let $\lambda=1$ ):

$$
\begin{gathered}
\operatorname{Per}\left(E_{s}\right)+\int_{E_{s}} s-g(x) d x \leq \operatorname{Per}\left(E_{s} \cup E_{s^{\prime}}\right)+\int_{E_{s} \cup E_{s^{\prime}}} s-g(x) d x, \\
\operatorname{Per}\left(E_{s^{\prime}}\right)+\int_{E_{s^{\prime}}} s^{\prime}-g(x) d x \leq \operatorname{Per}\left(E_{s} \cap E_{s^{\prime}}\right)+\int_{E_{s} \cap E_{s^{\prime}}} s^{\prime}-g(x) d x
\end{gathered}
$$

and summing both inequalities we get:

$$
\begin{aligned}
& \operatorname{Per}\left(E_{s}\right)+\operatorname{Per}\left(E_{s^{\prime}}\right)+\int_{E_{s}} s-g(x) d x+\int_{E_{s^{\prime}}} s^{\prime}-g(x) d x \\
& \leq \operatorname{Per}\left(E_{s} \cup E_{s^{\prime}}\right)+P\left(E_{s} \cap E_{s^{\prime}}\right)+\int_{E_{s} \cup E_{s^{\prime}}} s-g(x) d x+\int_{E_{s} \cap E_{s^{\prime}}} s^{\prime}-g(x) d x .
\end{aligned}
$$

Using (7), it follows that

$\int_{E_{s^{\prime}}} s^{\prime}-g(x) d x-\int_{E_{s} \cap E_{s^{\prime}}} s^{\prime}-g(x) d x \leq \int_{E_{s} \cup E_{s^{\prime}}} s-g(x) d x-\int_{E_{s}} s-g(x) d x$,

that is,

$$
\int_{E_{s^{\prime} \backslash E_{s}}} s^{\prime}-g(x) d x \leq \int_{E_{s^{\prime}} \backslash E_{s}} s-g(x) d x
$$

hence

$$
\left(s^{\prime}-s\right)\left|E_{s^{\prime}} \backslash E_{s}\right| \leq 0:
$$

it shows that $E_{s}^{\prime} \subseteq E_{s}$, up to a negligible set, as soon as $s^{\prime}>s$. 
In particular, it follows that $E_{s}$ is unique, except for at most countably many values of $s$. Indeed, we can introduce the sets $E_{s}^{+}=\bigcap_{s^{\prime}<s} E_{s^{\prime}}$ and $E_{s}^{-}=\bigcup_{s^{\prime}>s} E_{s^{\prime}}$, then one checks that $E_{s}^{+}$and $E_{s}^{-}$are respectively the largest and smallest solutions of $\left(R O F_{s}\right)^{1}$. There is uniqueness when the measure $\left|E_{s}^{+} \backslash E_{s}^{-}\right|=0$. But the sets $\left(E_{s}^{+} \backslash E_{s}^{-}\right), s \in \mathbb{R}$, are all disjoint, so that their measure must be zero except for at most countably many values.

Let us introduce the function:

$$
u(x)=\sup \left\{s \in \mathbb{R}: x \in E_{s}\right\} .
$$

We have that $u(x)>s$ if there exists $t>s$ with $x \in E_{t}$, so that in particular, $x \in E_{s}^{-}$; conversely, if $x \in E_{s}^{-}, x \in E_{s^{\prime}}$ for some $s^{\prime}>s$, so that $u(x)>s$ : $\{u>s\}=E_{s}^{-}$. (In the same way, we check $E_{s}^{+}=\{u \geq s\}$.)

Lemma 2.5. The function $u$ is the minimizer of $(R O F)$.

Proof. First of all, we check that $u \in L^{2}(\Omega)$. This is because

$$
\lambda \operatorname{Per}\left(E_{s} ; \Omega\right)+\int_{E_{s}} s-g(x) d x \leq 0
$$

(the energy of the emptyset), hence

$$
s\left|E_{s}\right| \leq \int_{E_{s}} g(x) d x
$$

It follows that

$$
\int_{0}^{M} s\left|E_{s}\right| d s \leq \int_{0}^{M} \int_{E_{s}} g(x) d x d s
$$

but $\int_{0}^{M} s\left|E_{s}\right| d s=\int_{E_{0}} \int_{0}^{u(x) \wedge M} s d s d x=\int_{E_{0}}(u(x) \wedge M)^{2} / 2 d x$ (using Fubini's theorem), while in the same way $\int_{0}^{M} \int_{E_{s}} g(x) d x d s=\int_{E_{0}}(u(x) \wedge M) g(x) d x$.

Hence

$$
\frac{1}{2} \int_{E_{0}}(u \wedge M)^{2} d x \leq \int_{E_{0}}(u \wedge M) g d x \leq\left(\int_{E_{0}}(u \wedge M)^{2} d x \int_{E_{0}} g^{2} d x\right)^{\frac{1}{2}}
$$

so that

$$
\int_{E_{0}}(u(x) \wedge M)^{2} d x \leq 4 \int_{E_{0}} g(x)^{2} d x
$$

\footnotetext{
${ }^{1}$ Observe that since the set $E_{s}$ are normally defined up to negligible sets, the intersections $E_{s}^{+}=\bigcap_{s^{\prime}<s} E_{s^{\prime}}$ might not be well-defined (as well as the unions $E_{s}^{-}$). A rigorous definition requires, actually, either to consider only countable intersections/unions, or to first choose a precise representative of each $E_{s}$, for instance the set $\left\{x \in E_{s}: \lim _{\rho \rightarrow 0}\left|E_{s} \cap B(x, \rho)\right| /|B(x, \rho)|=1\right\}$ of points of Lebesgue density 1 , which can be shown, for $E_{s}$ minimizing $\left(R O F_{s}\right)$, to be an open set.
} 
and sending $M \rightarrow \infty$ it follows that

$$
\int_{\{u>0\}} u(x)^{2} d x \leq 4 \int_{\{u>0\}} g(x)^{2} d x .
$$

In the same way, we can show that

$$
\int_{\{u<0\}} u(x)^{2} d x \leq 4 \int_{\{u<0\}} g(x)^{2} d x .
$$

This requires the observation that the set $\{-u>-s\}=\{u<s\}=\Omega \backslash E_{s}^{+}$is a minimizer of the problem

$$
\min _{E} \operatorname{Per}(E ; \Omega)+\int_{E} g(x)-s d x
$$

which easily follows from the fact that $\operatorname{Per}(E ; \Omega)=\operatorname{Per}(\Omega \backslash E ; \Omega)$ for any set of finite perimeter $E \subset \Omega$ : it follows that if we replace $g$ with $-g$ in $\left(R O F_{s}\right)$, then the function $u$ is replaced with $-u$.

We deduce from $(27)$ and $(28)$ that $u \in L^{2}(\Omega)$.

Let now $v \in B V(\Omega) \cap L^{2}(\Omega)$ : we have for any $M>0$,

$$
\int_{-M}^{M} \lambda \operatorname{Per}\left(E_{s}^{-} ; \Omega\right)+\int_{E_{s}^{-}} s-g(x) d x \leq \int_{-M}^{M} \lambda \operatorname{Per}(\{v>s\} ; \Omega)+\int_{\{v>s\}} s-g(x) d x
$$

since $E_{s}^{-}$is a minimizer for $\left(R O F_{s}\right)$. Notice that (using Fubini's Theorem again)

$$
\begin{aligned}
\int_{-M}^{M} \int_{\{v>s\}} s-g(x) d x & =\int_{\Omega} \int_{-M}^{M} \chi_{\{v>s\}}(x)(s-g(x)) d x \\
= & \frac{1}{2} \int_{\Omega}((v(x) \wedge M)-g(x))^{2}-((v(x) \wedge(-M))-g(x))^{2} d x,
\end{aligned}
$$

hence

$$
\int_{-M}^{M} \int_{\{v>s\}} s-g(x) d x+\int_{\Omega}(M+g(x))^{2} d x=\frac{1}{2} \int_{\Omega}(v(x)-g(x))^{2} d x+\mathcal{R}(v, M)
$$

where

$$
\begin{aligned}
\mathcal{R}(v, M)=\frac{1}{2}\left(\int_{\Omega}((v(x) \wedge\right. & M)-g(x))^{2}-(v(x)-g(x))^{2} d x \\
& \left.+\int_{\Omega}(-M-g(x))^{2}-((v(x) \wedge(-M))-g(x))^{2} d x\right)
\end{aligned}
$$

It suffices now to check that for any $v \in L^{2}(\Omega)$,

$$
\lim _{M \rightarrow \infty} \mathcal{R}(v, M)=0
$$


We leave it to the reader. From (29) and (30), we get that

$$
\begin{aligned}
& \lambda \int_{-M}^{M} \operatorname{Per}(\{u>s\} ; \Omega)+\frac{1}{2} \int_{\Omega}(u-g)^{2} d x+\mathcal{R}(u, M) \\
& \leq \lambda \int_{-M}^{M} \operatorname{Per}(\{v>s\} ; \Omega)+\frac{1}{2} \int_{\Omega}(v-g)^{2} d x+\mathcal{R}(v, M),
\end{aligned}
$$

sending $M \rightarrow \infty$ (and using the fact that both $u$ and $v$ are in $L^{2}$, so that $\mathcal{R}(\cdot, M)$ goes to 0$)$ we deduce

$$
\lambda J(u)+\frac{1}{2} \int_{\Omega}(u-g)^{2} d x \leq \lambda J(v)+\frac{1}{2} \int_{\Omega}(v-g)^{2} d x,
$$

that is, the minimality of $u$ for $(R O F)$.

We have proved the following result:

Proposition 2.6. A function $u$ solves $(R O F)$ if and only if for any $s \in \mathbb{R}$, the set $\{u>s\}$ solves $\left(R O F_{s}\right)$.

Normally, we should write "for almost any $s$ ", but as before by approximation it is easy to show that if it is true for almost all $s$, then it is true for all $s$.

This is interesting for several applications. It provides another way to solve problems such as (16) (through an unconstrained relaxation - but in fact both problems are relatively easy to solve). But most of all, it gives a lot of information on the level sets of $u$, as problems such as (16) have been studied thoroughly in the past 50 years.

The link between minimal surfaces and functions minimizing the total variation was first identified by De Giorgi and Bombieri, as a tool for the study of minimal surfaces.

Theorem 2.6 can be generalized easily to the following case: we should have that $u$ is a minimizer of

$$
\min _{u} J(u)+\int_{\Omega} G(x, u(x)) d x
$$

for some $G$ measurable in $x$, and convex, $C^{1}$ in $u$, if and only if for any $s \in \mathbb{R}$, $\{u>s\}$ minimizes

$$
\min _{E} \operatorname{Per}(E ; \Omega)+\int_{E} g(x, s) d x
$$

where $g(x, s)=\partial_{s} G(x, s)$. The case of nonsmooth $G$ is also interesting and has been studied by Chan and Esedoglu [25]. 


\subsubsection{A few explicit solutions}

The results in this section are simplifications of results which are found in $[5,4]$ (see also [3] for more results of the same kind).

Let us show how Theorem 2.6 can help build explicit solutions of $(R O F)$, in a few very simple cases. We consider the two following cases: $\Omega=\mathbb{R}^{2}$, and

i. $g=\chi_{B(0, R)}$ the characteristic of a ball;

ii. $g=\chi_{[0,1]^{2}}$ the characteristic of the unit square.

In both case, $g=\chi_{C}$ for some convex set $C$. First observe that obviously, $0 \leq u \leq 1$. As in the introduction, indeed, one easily checks that $u \wedge 1=\min \{u, 1\}$ has less energy than $u$. Another way is to observe that $E_{s}=\{u>s\}$ solves

$$
\min _{E} \lambda \operatorname{Per}(E)+\int_{E} s-\chi_{C}(x) d x
$$

but if $s>1, s-\chi_{C}$ is always positive so that $E=\emptyset$ is clearly optimal, while if $s<0, s-\chi_{C}$ is always negative so that $E=\mathbb{R}^{2}$ is optimal (and in this case the value is $-\infty)$.

Now, if $s \in(0,1), E_{s}$ solves

$$
\min _{E} \lambda \operatorname{Per}(E)-(1-s)|E \cap C|+s|E \backslash C| .
$$

Let $P$ be a half-plane containing $C$ : observe that $\operatorname{Per}(E \cap P) \leq \operatorname{Per}(E)$, since we replace the part of a boundary outside of $P$ with a straight line on $\partial P$, while $|(E \cap P) \backslash C| \leq|E \backslash C|$ : hence, the set $E \cap P$ has less energy than $E$, see Fig. 5. Hence $E_{s} \subset P$. As $C$, which is convex, is the intersection of all half-planes containing it, we deduce that $E_{s} \subset C$. (In fact, this is true in any dimension for any convex set C.)

We see that the problem for the level sets $E_{s}(31)$ becomes:

$$
\min _{E \subset C} \lambda \operatorname{Per}(E)-(1-s)|E|
$$

The characteristic of a ball If $g=\chi_{B(0, R)}$, the ball of radius $R$ (in $\mathbb{R}^{2}$ ), we see that thanks to the isoperimetric inequality (13),

$$
\lambda \operatorname{Per}(E)-(1-s)|E| \geq \lambda 2 \sqrt{\pi} \sqrt{|E|}-(1-s)|E|,
$$

and for $|E| \in[0,|B(0, R)|]$, the right-hand side is minimal only if $|E|=0$ or $|E|=|B(0, R)|$, with value 0 in the first case, $2 \lambda \pi R-(1-s) \pi R^{2}$ in the second. 


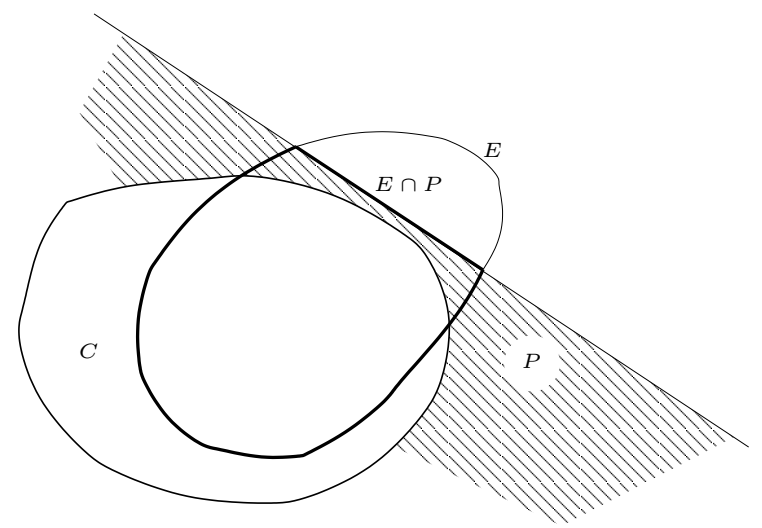

Figure 5: $E \cap P$ has less energy than $E$

Since for these two choices, the isoperimetric inequality is an equality, we deduce that the min in (32) is actually attained by $E=\emptyset$ if $s \geq 1-2 \lambda / R$, and $E=B(0, R)$ if $s \leq 1-2 \lambda / R$. Hence the solution is

$$
u=\left(1-\frac{2 \lambda}{R}\right)^{+} \chi_{B(0, R)}
$$

for any $\lambda>0$ (here $x^{+}=\max \{x, 0\}$ is the positive part of the real number $x$ ). In fact, this result holds in all dimension (with 2 replaced with $N$ in the expression). See also [52].

The characteristic of a square The case of the characteristic of a square is a bit different. The level sets $E_{s}$ need to solve (32). From the Euler-Lagrange equation (see also (26)) it follows that the curvature of $\partial E_{s} \cap C$ is $(1-s) / \lambda$. An accurate study (see also $[5,4,42]$ ) shows that, if we define (for $C=[0,1]^{2}$ )

$$
C_{R}=\bigcup_{x: B(x, R) \subset C} B(x, R)
$$

and let $R^{*}$ be the value of $R$ for which $P\left(C_{R}\right) /\left|C_{R}\right|=1 / R$, then for any $s \in[0,1]$,

$$
E_{s}= \begin{cases}\emptyset & \text { if } s \geq 1-\frac{\lambda}{R^{*}} \\ C_{\lambda /(1-s)} & \text { if } s \leq 1-\frac{\lambda}{R^{*}}\end{cases}
$$




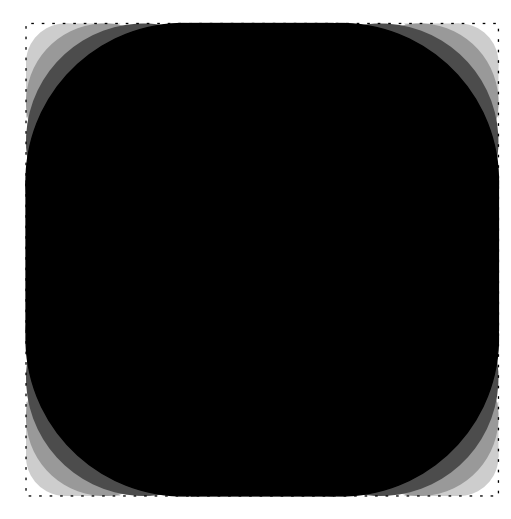

Figure 6: Solution $u$ for $g=\chi_{[0,1]^{2}}$

while letting $v(x)=1 / \mathbb{R}^{*}$ if $x \in C_{R^{*}}$, and $1 / R$ if $x \in \partial C_{R}$, we find

$$
u=(1-\lambda v(x))^{+} \text {, }
$$

see Fig. 6.

\subsubsection{The discontinuity set}

We now show that the jump set of $u_{\lambda}$ is always contained in the jump set of $g$. More precisely, we will describe shortly the proof of the following result, which was first proved in [20]:

Theorem 5 (Caselles-C-Novaga). Let $g \in B V(\Omega) \cap L^{\infty}(\Omega)$ and $u$ solve $(R O F)$. Then $J_{u} \subseteq J_{g}$ (up to a set of zero $\mathcal{H}^{N-1}$-measure).

Hence, if $g$ is already a $B V$ function, the Rudin-Osher-Fatemi denoising will never produce new discontinuities.

First, we use the following important regularity result, from the theory of minimal surfaces $[40,6]$ :

Proposition 2.7. Let $g \in L^{\infty}(\Omega), s \in \mathbb{R}$, and $E_{s}$ be a minimzer of $\left(R O F_{s}\right)$. Then $\Sigma=\partial E_{s} \backslash \partial^{*} E_{s}$ is a closed set of Hausdorff dimension at most $N-8$, while near each $x \in \partial^{*} E_{s}, \partial^{*} E_{s}$ is locally the graph of a function of class $W^{2, q}$ for all $q<+\infty$ (and, in dimension $N=2, W^{2, \infty}=C^{1,1}$ ).

It means that outside of a very small set (which is empty if $N \leq 7$ ), then the boundary of $E_{s}$ is $C^{1}$, and the normal is still differentiable but in a weaker sense. We now can show Theorem 5 . 
Proof. The jump set $J_{u}$ is where several level sets intersect: if we choose $\left(s_{n}\right)_{n \geq 1}$ a dense sequence in $\mathbb{R}^{N}$ of levels such that $E_{n}=E_{s_{n}}=\left\{u>s_{n}\right\}$ are finite perimeter sets each solving $\left(R O F_{s_{n}}\right)$, we have

$$
J_{u}=\bigcup_{n \neq m}\left(\partial^{*} E_{n} \cap \partial^{*} E_{m}\right) .
$$

Hence it is enough to show that for any $n, m$ with $n \neq m$,

$$
\mathcal{H}^{N-1}\left(\left(\partial^{*} E_{n} \cap \partial^{*} E_{m}\right) \backslash J_{g}\right)=0
$$

which precisely means that $\partial^{*} E_{n} \cap \partial^{*} E_{m} \subseteq J_{g}$ up to a negligible set. Consider thus a point $x^{0} \in \partial^{*} E_{n} \cap \partial^{*} E_{m}$ such that (without loss of generality we let $x^{0}=0$ ):

i. up to a change of coordinates, in a small neighborhood $\left\{x=\left(x_{1}, \ldots, x_{N}\right)=\right.$ $\left.\left(x^{\prime}, x_{N}\right):\left|x^{\prime}\right|<R,\left|x_{N}\right|<R\right\}$ of $x^{0}=0$, the sets $E_{n}$ and $E_{m}$ coincide respectively to $\left\{x_{N}<v_{n}\left(x^{\prime}\right)\right\}$ and $\left\{x_{N}<v_{m}\left(x^{\prime}\right)\right\}$, with $v_{n}$ and $v_{m}$ in $W^{2, q}\left(B^{\prime}\right)$ for all $q<+\infty$, where $B^{\prime}=\left\{\left|x^{\prime}\right|<R\right\}$.

ii. the measure of the contact set $\left\{x^{\prime} \in B^{\prime}: v_{n}\left(x^{\prime}\right)=v_{m}\left(x^{\prime}\right)\right\}$ is positive.

We assume without loss of generality that $s_{n}<s_{m}$, so that $E_{m} \subseteq E_{n}$, hence $v_{n} \geq v_{m}$ in $B^{\prime}$.

From $\left(R O F_{s}\right)$, we see that the function $v_{l}, l \in\{n, m\}$, must satisfy

$$
\begin{aligned}
\int_{B^{\prime}} & {\left[\sqrt{1+\left|\nabla v_{l}\left(x^{\prime}\right)\right|^{2}}+\int_{0}^{v_{l}\left(x^{\prime}\right)}\left(s_{l}-g\left(x^{\prime}, x_{N}\right)\right) d x_{N}\right] d x^{\prime} } \\
& \leq \int_{B^{\prime}}\left[\sqrt{1+\left|\nabla v_{l}\left(x^{\prime}\right)+t \phi\left(x^{\prime}\right)\right|^{2}}+\int_{0}^{v_{l}\left(x^{\prime}\right)+t \phi\left(x^{\prime}\right)}\left(s_{l}-g\left(x^{\prime}, x_{N}\right)\right) d x_{N}\right] d x^{\prime}
\end{aligned}
$$

for any smooth $\phi \in C_{c}^{\infty}\left(B^{\prime}\right)$ and any $t \in \mathbb{R}$ small enough, so that the perturbation remains in the neighborhood of $x^{0}$ where $E_{s_{n}}$ and $E_{s_{m}}$ are subgraphs. Here, the first integral corresponds to the perimeter of the set $\left\{x_{N}<v_{l}\left(x^{\prime}\right)+t \phi\left(x^{\prime}\right)\right\}$ and the second to the volume integral in $\left(R O F_{s}\right)$.

We consider $\phi \geq 0$, and compute

$$
\begin{aligned}
\lim _{\substack{t \rightarrow 0, t \\
t>0}} \frac{1}{t}\left(\int_{B^{\prime}}\right. & {\left[\sqrt{1+\left|\nabla v_{l}\left(x^{\prime}\right)+t \phi\left(x^{\prime}\right)\right|^{2}}+\int_{0}^{v_{l}\left(x^{\prime}\right)+t \phi\left(x^{\prime}\right)}\left(s_{l}-g\left(x^{\prime}, x_{N}\right)\right) d x_{N}\right] d x^{\prime} } \\
& \left.-\int_{B^{\prime}}\left[\sqrt{1+\left|\nabla v_{l}\left(x^{\prime}\right)\right|^{2}}+\int_{0}^{v_{l}\left(x^{\prime}\right)}\left(s_{l}-g\left(x^{\prime}, x_{N}\right)\right) d x_{N}\right] d x^{\prime}\right) \geq 0,
\end{aligned}
$$


we find that $v_{l}$ must satisfy

$$
\int_{B^{\prime}} \frac{\nabla v_{l}\left(x^{\prime}\right) \cdot \nabla \phi\left(x^{\prime}\right)}{\sqrt{1+\left|\nabla v_{l}\left(x^{\prime}\right)\right|^{2}}}+\left(s_{l}-g\left(x^{\prime}, v_{l}\left(x^{\prime}\right)+0\right)\right) \phi\left(x^{\prime}\right) d x^{\prime} \geq 0 .
$$

Integrating by parts, we find

$$
\left.-\operatorname{div} \frac{\nabla v_{l}}{\sqrt{1+\left|\nabla v_{l}\right|^{2}}}+s_{l}-g\left(x^{\prime}, v_{l}\left(x^{\prime}\right)+0\right)\right) \geq 0,
$$

and in the same way, taking this time the limit for $t<0$, we find that

$$
\left.-\operatorname{div} \frac{\nabla v_{l}}{\sqrt{1+\left|\nabla v_{l}\right|^{2}}}+s_{l}-g\left(x^{\prime}, v_{l}\left(x^{\prime}\right)-0\right)\right) \leq 0 .
$$

Both (33) and (34) must hold almost everywhere in $B^{\prime}$. At the contact points $x^{\prime}$ where $v_{n}\left(x^{\prime}\right)=v_{m}\left(x^{\prime}\right)$, since $v_{n} \geq v_{m}$, we have $\nabla v_{n}\left(x^{\prime}\right)=\nabla v_{m}\left(x^{\prime}\right)$, while $D^{2} v_{n}\left(x^{\prime}\right) \geq D^{2} v_{m}\left(x^{\prime}\right)$ at least at a.e. contact point $x^{\prime}$. In particular, it follows

$$
-\operatorname{div} \frac{\nabla v_{n}}{\sqrt{1+\left|\nabla v_{n}\right|^{2}}}\left(x^{\prime}\right) \leq-\operatorname{div} \frac{\nabla v_{m}}{\sqrt{1+\left|\nabla v_{m}\right|^{2}}}\left(x^{\prime}\right)
$$

If the contact set $\left\{v_{n}=v_{m}\right\}$ has positive $((N-1)$-dimensional) measure, we can find a contact point $x^{\prime}$ such that (35) holds, as well as both (33) and (34), for both $l=n$ and $l=m$. It follows

$$
g\left(x^{\prime}, v_{n}\left(x^{\prime}\right)+0\right)-s_{n} \leq g\left(x^{\prime}, v_{m}\left(x^{\prime}\right)-0\right)-s_{m}
$$

and denoting $x_{N}$ the common value $v_{n}\left(x^{\prime}\right)=v_{m}\left(x^{\prime}\right)$, we get

$$
0<s_{m}-s_{n} \leq g\left(x^{\prime}, x_{N}-0\right)-g\left(x^{\prime}, x_{N}+0\right)
$$

It follows that $x=\left(x^{\prime}, x_{N}\right)$ must be a jump point of $g$ (with the values below $x_{N}$ larger than the values above $x_{N}$, so that the jump occurs in the same direction for $g$ and $u$ ). This concludes the proof: the possible jump points outside of $J_{g}$ are negligible for the $(N-1)$-dimensional measure. The precise meaning of $g\left(x^{\prime}, x_{N} \pm 0\right)$ and the relationship to the jump of $g$ is rigorous for a.e. $x^{\prime}$, see the "slicing properties of $B V$ functions" in [7].

Remark We have also proved that for almost all points in $J_{u}$, we have $\nu_{u}=\nu_{g}$, that is, the orientation of the jumps of $u$ and $g$ are the same, which is intuitively obvious. 


\subsubsection{Regularity}

The same idea, based on the control on the curvature of the level sets which is provided by $\left(R O F_{s}\right)$, yields further continuity results for the solutions of $(R O F)$. The following theorems are proved in [21]:

Theorem 6 (Caselles-C-Novaga). Assume $N \leq 7$ and let $u$ be a minimizer. Let $A \subset \Omega$ be an open set and assume that $g \in C^{0, \beta}(A)$ for some $\beta \in[0,1]$. Then, also $u \in C^{0, \beta}\left(A^{\prime}\right)$ for any $A^{\prime} \subset \subset A$.

Here, $A^{\prime} \subset \subset A$ means that $\overline{A^{\prime}} \subset A$. The proof of this result is quite complicated and we refer to [21]. The reason for restriction on the dimension is clear from Proposition 2.7, since we need here in the proof that $\partial E_{s}$ is globally regular for all $s$. The next result is proved more easily:

Theorem 7 (Caselles-C-Novaga). Assume $N \leq 7$ and $\Omega$ is convex. Let $u$ solve $(R O F)$, and suppose $g$ is uniformly continuous with modulus of continuity $\omega$ (that is, $|g(x)-g(y)| \leq \omega(|x-y|)$ for all $x, y$, with $\omega$ continuous, nondecreasing, and $\omega(0)=0)$. Then $u$ has the same modulus of continuity.

For instance, if $g$ is globally Lipschitz, then also $u$ is with same constant.

Proof. We only sketch the proof: by approximation we can assume that $\Omega$ is smooth and uniformly convex, and, as well, that $g$ is smooth up to the boundary.

We consider two levels $s$ and $t>s$ and the corresponding level sets $E_{s}$ and $E_{t}$. Let $\delta=\operatorname{dist}\left(\Omega \cap \partial E_{s}, \Omega \cap \partial E_{t}\right)$ be the distance between the level sets $s$ and $t$ of $u$. The strict convexity of $\Omega$ and the fact that $\partial E_{s}$ are smooth, and orthogonal to the boundary (because $z \cdot \nu=0$ on $\partial \Omega$ in (26)), imply that this minimal distance cannot be reached by points on the boundary $\partial \Omega$, but that there exists $x_{s} \in \partial E_{s} \cap \Omega$ and $x_{t} \in \partial E_{t} \cap \Omega$, with $\left|x_{s}-x_{t}\right|=\delta$. We can also exclude the case $\delta=0$, by arguments similar to the previous proof.

Let $e=\left(x_{s}-x_{t}\right) / \delta$. This must be the outer normal to both $\partial E_{t}$ and $\partial E_{s}$, respectively at $x_{t}$ and $x_{s}$.

The idea is to "slide" one of the sets until it touches the other, in the direction $e$. We consider, for $\varepsilon>0$ small, $\left(E_{t}+(\delta+\varepsilon) e\right) \backslash \bar{E}_{s}$, and a connected component $W_{\varepsilon}$ which contains $x_{s}$ on its boundary. We then use $\left(R O F_{t}\right)$, comparing $E_{t}$ with 
$E_{t} \backslash\left(W_{\varepsilon}-(\delta+\varepsilon) e\right)$ and $E_{s}$ with $E_{s} \cup W_{\varepsilon}$ :

$$
\begin{aligned}
\operatorname{Per}\left(E_{t} ; \Omega\right) & +\int_{E_{t}}(t-g(x)) d x \\
& \leq \operatorname{Per}\left(E_{t} \backslash\left(W_{\varepsilon}-(\delta+\varepsilon) e\right) ; \Omega\right)+\int_{E_{t} \backslash\left(W_{\varepsilon}-(\delta+\varepsilon) e\right)}(t-g(x)) d x \\
\operatorname{Per}\left(E_{s} ; \Omega\right) & +\int_{E_{s}}(s-g(x)) d x \\
& \leq \operatorname{Per}\left(E_{s} \cup W_{\varepsilon} ; \Omega\right)+\int_{E_{s} \cup W_{\varepsilon}}(s-g(x)) d x .
\end{aligned}
$$

Now, if we let $L_{t}=\mathcal{H}^{N-1}\left(\partial W_{\varepsilon} \backslash \partial E_{s}\right)$ and $L_{s}=\mathcal{H}^{N-1}\left(\partial W_{\varepsilon} \cap \partial E_{s}\right)$, we have that

$$
\operatorname{Per}\left(E_{t} \backslash\left(W_{\varepsilon}-(\delta+\varepsilon) e\right), \Omega\right)=\operatorname{Per}\left(E_{t}, \Omega\right)-L_{t}+L_{s}
$$

and

$$
\operatorname{Per}\left(E_{s} \cup W_{\varepsilon}, \Omega\right)=\operatorname{Per}\left(E_{s}, \Omega\right)+L_{t}-L_{s},
$$

so that, summing both equations in (36), we deduce

$$
\int_{W_{\varepsilon}-(\delta+\varepsilon) e}(t-g(x)) d x \leq \int_{W_{\varepsilon}}(s-g(x)) d x .
$$

Hence,

$$
(t-s)\left|W_{\varepsilon}\right| \leq \int_{W_{\varepsilon}}(g(x+(\delta+\varepsilon) e)-g(x)) d x \leq\left|W_{\varepsilon}\right| \omega(\delta+\varepsilon) .
$$

Dividing both sides by $\left|W_{\varepsilon}\right|>0$ and sending then $\varepsilon$ to zero, we deduce

$$
t-s \leq \omega(\delta) .
$$

The regularity of $u$ follows. Indeed, if $x, y \in \Omega$, with $u(x)=t>u(y)=s$, we find $|u(x)-u(y)| \leq \omega(\delta) \leq \omega(|x-y|)$ since $\delta \leq|x-y|, \delta$ being the minimal distance between the level surface of $u$ through $x$ and the level surface of $u$ through $y$.

\section{Algorithmic issues}

\subsection{Discrete problem}

To simplify, we let now $\Omega=(0,1)^{2}$ and we will consider here a quite straightforward discretization of the total variation in $2 D$, as

$$
T V_{h}(u)=h^{2} \sum_{i, j} \frac{\sqrt{\left|u_{i+1, j}-u_{i, j}\right|^{2}-\left|u_{i, j+1}-u_{i, j}\right|^{2}}}{h} .
$$


Here in the sum, the differences are replaced by 0 when one of the points is not on the grid. The matrix $\left(u_{i, j}\right)$ is our discrete image, defined for instance for $i, j=1, \ldots, N$, and $h=1 / N$ is the discretization step: $T V_{h}$ is therefore an approximation of the $2 D$ total variation of a function $u \in L^{1}\left((0,1)^{2}\right)$ at scale $h>0$.

It can be shown in many ways that (37) is a "correct" approximation of the Total Variation $J$ introduced previously and we will skip this point. The most simple result is as follows:

Proposition 3.1. Let $\Omega=(0,1)^{2}, p \in[1,+\infty)$, and $G: L^{p}(\Omega) \rightarrow \mathbb{R}$ a continuous functional such that $\lim _{c \rightarrow \infty} G(c+u)=+\infty$ for any $u \in L^{p}(\Omega)$ (this coerciveness assumption is just to ensure the existence of a solution to the problem, and other situations could be considered). Let $h=1 / N>0$ and $u^{h}=\left(u_{i, j}\right)_{1 \leq i, j \leq N}$, identified with $u^{h}(x)=\sum_{i, j} \chi_{((i-1) h, i h) \times((j-1) h, j h)}(x)$, be the solution of

$$
\min _{u^{h}} T V_{h}\left(u^{h}\right)+G\left(u^{h}\right) .
$$

Then, there exists $u \in L^{p}(\Omega)$ such that some subsequence $u^{h_{k}} \rightarrow u$ as $k \rightarrow \infty$ in $L^{1}(\Omega)$, and $u$ is a minimizer in $L^{p}(\Omega)$ of

$$
J(u)+G(u) .
$$

But more precise results can be shown, including with error bounds, see for instance recent works [50, 45].

In what follows, we will choose $h=1$ (introducing $h$ yields in general to straightforward changes in the other parameters). Given $u=\left(u_{i, j}\right)$ a discrete image $(1 \geq i, j, \geq N)$, we introduce the discrete gradient

$$
(\nabla u)_{i, j}=\left(\begin{array}{c}
\left(D_{x}^{+} u\right)_{i, j} \\
\left(D_{y}^{+} u\right)_{i, j}
\end{array}\right)=\left(\begin{array}{c}
u_{i+1, j}-u_{i, j} \\
u_{i, j+1}-u_{i, j}
\end{array}\right)
$$

except at the boundaries: if $i=N,\left(D_{x}^{+} u\right)_{N, j}=0$, and if $j=N,\left(D_{y}^{+} u\right)_{i, N}=0$. Let $X=\mathbb{R}^{N \times N}$ be the vector space where $u$ lives, then $\nabla$ is a linear map from $X$ to $Y=X \times X$, and (if we endow both spaces with the standard Euclidean scalar product), its adjoint $\nabla^{*}$, denoted by - div, and defined by

$$
\langle\nabla u, p\rangle_{Y}=\left\langle u, \nabla^{*} p\right\rangle_{X}=-\langle u, \operatorname{div} p\rangle_{X}
$$

for any $u \in X$ and $p=\left(p_{i, j}^{x}, p_{i, j}^{y}\right) \in Y$, is given by the following formulas

$$
(\operatorname{div} p)_{i, j}=p_{i, j}^{x}-p_{i-1, j}^{x}+p_{i, j}^{y}-p_{i, j-1}^{y}
$$


for $2 \leq i, j \leq N-1$, and the difference $p_{i, j}^{x}-p_{i-1, j}^{x}$ is replaced with $p_{i, j}^{x}$ if $i=1$, and with $-p_{i-1, j}^{x}$ if $i=N$, while $p_{i, j}^{y}-p_{i, j-1}^{y}$ is replaced with $p_{i, j}^{y}$ if $j=1$ and with $-p_{i, j-1}^{y}$ if $j=N$.

We will focus on algorithms for solving the discrete problem

$$
\min _{u \in X} \lambda\|\nabla u\|_{2,1}+\frac{1}{2}\|u-g\|^{2}
$$

where $\|p\|_{2,1}=\sum_{i, j} \sqrt{\left(p_{i, j}^{x}\right)^{2}+\left(p_{i, j}^{y}\right)^{2}}$. In this section and all what follows, $J(\cdot)$ will now denote the discrete total variation $J(u)=\|\nabla u\|_{2,1}$ (and we will not speak anymore of the continuous variation introduced in the previous section). The problem (38) can also be written in the more general form

$$
\min _{u \in X} F(A u)+G(u)
$$

where $F: Y \rightarrow \mathbb{R}_{+}$and $G: X \rightarrow \mathbb{R}$ are convex functions and $A: X \rightarrow Y$ is a linear operator (in the discretization of $(R O F), A=\nabla, F(p)=\|p\|_{2,1}, G(u)=\|u-g\|_{2 \lambda}^{2}$ ).

It is essential here that $F, G$ are convex, since we will focus on techniques of convex optimization which can produce quite efficient algorithms for problems of the form (39), provided $F$ and $G$ have a simple structure.

\subsection{Basic convex analysis - Duality}

Before detailing a few numerical methods to solve (38) let us recall the basics of convex analysis in finite-dimensional spaces (all the results we state now are true in a more general setting, and the proofs in the Hilbertian framework are the same). We refer of course to $[67,31]$ for more complete information.

\subsubsection{Convex functions - Legendre-Fenchel conjugate}

Let $X$ be a finite-dimensional, Euclidean space (or a Hilbert space). Recall that a subset $C \subset X$ of $X$ is said to be convex if and only if for any $x, x^{\prime} \in C$, the segment $\left[x, x^{\prime}\right] \subset C$, that is, for any $t \in[0,1]$,

$$
t x+(1-t) x^{\prime} \in C .
$$

Let us now introduce a similar definition for functions:

Definition 3.2. We say that the function $F: X \rightarrow[-\infty,+\infty]$ is 
- convex if and only if for any $x, x^{\prime} \in X, t \in[0,1]$,

$$
F\left(t x+(1-t) x^{\prime}\right) \leq t F(x)+(1-t) F\left(x^{\prime}\right),
$$

- proper if and only if $F$ is not identically $-\infty$ or $+\infty^{2}$

- lower-semicontinuous (l.s.c.) if and only for any $x \in X$ and $\left(x_{n}\right)_{n}$ a sequence converging to $x$,

$$
F(x) \leq \liminf _{n \rightarrow \infty} F\left(x_{n}\right) .
$$

We let $\Gamma^{0}(X)$ be the set of all convex, proper, l.s.c. functions on $X$.

It is well known, and easy to show, that if $F$ is twice differentiable at any $x \in X$, then it is convex if and only if $D^{2} F(x) \geq 0$ at any $x \in X$, in the sense that for any $x, y, \sum_{i, j} \partial_{i, j}^{2} F(x) y_{i} y_{j} \geq 0$. If $F$ is of class $C^{1}$, one has that $F$ is convex if and only if

$$
\langle\nabla F(x)-\nabla F(y), x-y\rangle \geq 0
$$

for any $x, y \in X$.

For any function $F: X \rightarrow[-\infty,+\infty]$, we define the domain

$$
\operatorname{dom} F=\{x \in X: F(x)<+\infty\}
$$

(which is always a convex set if $F$ is) and the epigraph

$$
\text { epi } F=\{(x, t) \in X \times \mathbb{R}: t \geq F(x)\} \text {, }
$$

which is convex if and only if $F$ is.

Then, it is well-known that $F$ is l.s.c. if and only if for any $\lambda \in \mathbb{R},\{F \leq \lambda\}$ is closed, if and only if epi $F$ is closed in $X \times \mathbb{R}$. [Indeed, if $F$ is l.s.c. and $\left(x_{n}\right) \in\{F \leq$ $\lambda\}$ converges to $x$, then $F(x) \leq \liminf _{n} F\left(x_{n}\right) \leq \lambda$, so that $\{F \leq \lambda\}$ is closed; if this set is closed and $\left(x_{n}, t_{n}\right)_{n}$ is a sequence if epi $F$ converging to $(x, t)$, then for any $\lambda>t,(x, t) \in\{F \leq \lambda\}$, that is $F(x) \leq \lambda$, so that $F(x) \leq t$ and $(x, t) \in$ epi $F$; and if epi $F$ is closed and $\left(x_{n}\right)_{n} \rightarrow x$, for any $t>\liminf _{n} F\left(x_{n}\right)$ there exists a subsequence $\left(x_{n_{k}}\right)$ such that $\left(x_{n_{k}}, t\right) \in$ epi $F$ for each $k$, so that $(x, t) \in$ epi $F$ hence $F(x) \leq t$. We deduce (41).]

Hence, we have $F \in \Gamma^{0}$ if and only if epi $F$ is closed, convex, nonempty and differs from $X \times \mathbb{R}$.

\footnotetext{
${ }^{2}$ notice that if $F$ is convex, $F \equiv-\infty$ if and only if there exists $x \in X$ s.t. $F(x)=-\infty$, so that a proper convex function has values in $\mathbb{R} \cup\{+\infty\}$.
} 
Another standard fact is that in finite dimension, any convex $F$ is locally Lipschitz in the interior of its domain.

Definition 3.3 (Legendre-Fenchel conjugate). We define the Legendre-Fenchel conjugate $F^{*}$ of $F$ for any $p \in X$ by

$$
F^{*}(p)=\sup _{x \in X}\langle p, x\rangle-F(x) .
$$

It is obvious that $F^{*}$ is convex, l.s.c. (as a supremum of linear, continuous functions). It is also proper if $F$ is convex and proper, as we will soon see: hence it maps $\Gamma^{0}$ into itself (and in fact, onto).

The following is the most classical and fundamental result of convex duality:

Theorem 8. Let $F \in \Gamma^{0}$ : then $F^{* *}=F$.

Before proving the theorem, let us state an important separation result on which it relies:

Theorem 9. Let $X$ be an Euclidean space, $C \subset X$ be a closed convex set, and $x \notin C$. Then there exists a closed hyperplane separating strictly $x$ and $C$, that is: there exists $p \in X$ and $\alpha \in \mathbb{R}$ such that

$$
\langle p, x\rangle>\alpha \geq\langle p, z\rangle
$$

for any $z \in C$.

This result is in its most general form a consequence of the Hahn-Banach theorem, however, in the finite dimensional, or Hilbert setting, its proof is much easier than in the general setting:

Proof. Let $\bar{x} \in C$ be the projection of $x$ onto $C$ :

$$
\bar{x}=\arg \min \left\{\left\|x-x^{\prime}\right\|^{2}: x^{\prime} \in C\right\} .
$$

The proof that it exists is standard and relies on the "parallelogram identity" $\|a+b\|^{2}+\|a-b\|^{2}=2\left(\|a\|^{2}+\|b\|^{2}\right)$, and the fact that $X$ is complete.

Let $p=x-\bar{x}$. We know that for any $z \in C$,

$$
\langle x-\bar{x}, z-\bar{x}\rangle \leq 0
$$

as is deduced from a straightforward first variation argument. Hence

$$
\langle p, z\rangle \leq \alpha=\langle p, \bar{x}\rangle
$$


On the other hand,

$$
\langle p, x\rangle=\langle p, \bar{x}\rangle+\|p\|^{2}>\alpha .
$$

Now we can prove Theorem (8):

Proof. First, for any $x$ and $p, F^{*}(p) \geq\langle p, x\rangle-F(x)$, so that $\langle p, x\rangle-F^{*}(p) \leq F(x)$, and taking the sup over $p$ we get that $F^{* *}(x) \leq F(x)$. Hence we need to show the opposite inequality.

To simplify, we prove it only in case $\operatorname{dom} F=X$. If $\operatorname{dom} F \neq X$, then it is easy to approximate $F$ as a sup of functions $\left(F_{\delta}\right)_{\delta>0}$ in $\Gamma^{0}$ (for instance, the MoreauYosida regularizations $\left.F_{\delta}(x)=\min _{y} F(y)+\|x-y\|^{2} /(2 \delta) \leq F(x)\right)$ and recover the result.

Let now $(x, t) \notin$ epi $F$, which is closed and convex. By the separation theorem, there exists $p, s, \alpha$ such that

$$
\langle p, x\rangle+s t>\alpha \geq\langle p, z\rangle+s u
$$

for any $(z, u) \in \operatorname{epi} F$. Sending $u \rightarrow+\infty$ we see that $s \leq 0$. On the other hand, since we have assumed $\operatorname{dom} F=X, x \in \operatorname{dom} F$ and $t<F(x)$, so that

$$
\langle p, x\rangle+s t>\alpha \geq\langle p, x\rangle+s F(x),
$$

and it follows $s>0$.

Now for any $z$,

$$
\left\langle\frac{p}{s}, x\right\rangle+t<\frac{\alpha}{s} \leq\left\langle\frac{p}{s}, z\right\rangle+F(z)
$$

we deduce that $\left\langle\frac{p}{s}, x\right\rangle+t<-F^{*}(-p / s)$, so that $t<F^{* *}(x)$. It follows that $F(x) \leq F^{* *}(x)$.

\subsubsection{Subgradient}

The definition is already given in Definition 2.2. Now $F$ is a convex function defined on a finite dimensional space $X$.

Definition 3.4. For $x \in X$,

$$
\partial F(x)=\{p \in X: F(y) \geq F(x)+\langle p, y-x\rangle \forall y \in \operatorname{dom} F\}
$$

and $\operatorname{dom} \partial F=\{x: \partial F(x) \neq \emptyset\} \subset \operatorname{dom} F$. 
If $F$ is differentiable at $x$, then $\partial F(x)=\{\nabla F(x)\}$.

Now, for any $p, x,\langle p, x\rangle \leq F(x)+F^{*}(p)$. But $p \in \partial F(x)$ implies that

$$
\langle p, x\rangle-F(x) \geq\langle p, y\rangle-F(y)
$$

for all $y$, hence

$$
\langle p, x\rangle-F(x) \geq F^{*}(p) .
$$

Hence, one deduces the Legendre-Fenchel identity:

Proposition 3.5. For any $F$ convex, $p \in \partial F(x)$ if and only if

$$
\langle p, x\rangle-F(x)=F^{*}(p) .
$$

Moreover if $F \in \Gamma^{0}$, so that $F^{* *}=F$, then this is equivalent to $x \in \partial F^{*}(p)$.

We have the following obvious proposition:

Proposition 3.6. Let $F$ be convex, then $x \in \arg \min _{X} F$ if and only if $0 \in \partial F(x)$.

Proof. indeed, this is equivalent to $F(y) \geq F(x)+\langle 0, y-x\rangle$ for all $y$.

The following trickier to show, but we skip the proof:

Proposition 3.7. Let $F, G$ be convex and assume $\operatorname{int}(\operatorname{dom} G) \cap \operatorname{dom} F \neq \emptyset$ : then $\partial(F+G)=\partial F+\partial G$.

The inclusion $\partial(F+G) \supset \partial F+\partial G$ always holds. For a proof, see [31]. The condition that $\operatorname{int}(\operatorname{dom} G) \cap \operatorname{dom} F \neq \emptyset$ (which is always true, for instance, if $\operatorname{dom} G=X$ ), is way too strong in finite dimension, where one may just assume that the relative interior of the domains of $G$ and $F$ is not empty [67]. If not, the result might not be true, as shows the example where $F(x)=+\infty$ if $x<0,-\sqrt{x}$ if $x \geq 0$, and $G(x)=F(-x)$.

Monotonicity The subgradient of a convex function $F$ is an example of "monotone" operator (in the sense of Minty): clearly from the definition we have for any $x, y$ and any $p \in \partial F(x), q \in \partial F(y)$,

$$
\langle p-q, x-y\rangle \geq 0
$$

(Just sum the two inequalities $F(y) \geq F(x)+\langle p, y-x\rangle$ and $F(x) \geq F(y)+$ $\langle q, x-y\rangle$.) Compare with (42). This is an essential property, as numerous algorithms have been designed (mostly in the 70's) to find the zeroes of monotone operators and their numerous variants. Also, a quite complete theory is available for defining and describing the flow of such operators [70, 19]. 


\subsubsection{The dual of $(R O F)$}

We now can easily derive the "dual" problem of (38) (and, in fact, also of (ROF) since everything we will write here also holds in the Hilbertian setting).

Recall that $J$ denotes now the discrete total variation introduced in (38). Let $u$ be a minimizer: from Propositions 3.6 and 3.7, we have

$$
0 \in \partial\left(\lambda J+\frac{1}{2}\|\cdot-g\|^{2}\right)(u)=\lambda \partial J(u)+u-g .
$$

(We recover in the discrete setting the same Euler-Lagrange equation as in the continuous setting, see (25).)

Hence: $(g-u) / \lambda \in \partial J(u)$, hence from Proposition 3.5, $u \in \partial J^{*}((g-u) / \lambda)$, hence $v=(g-u) / \lambda$ solves

$$
0 \in v-\frac{g}{\lambda}+\frac{1}{\lambda} \partial J^{*}(v)
$$

which is exactly the equation which characterizes the minimality for

$$
\min _{v} \frac{1}{2}\left\|v-\frac{g}{\lambda}\right\|^{2}+\frac{1}{\lambda} J^{*}(v) .
$$

Now, what is $J^{*}$ ? This is quite simple: $J$ can (as in the continuous setting) be defined by duality, indeed,

$$
\begin{aligned}
J(u)=\|\nabla u\|_{2,1}= & \sup \left\{\langle\xi, \nabla u\rangle_{Y}:\left|\xi_{i, j}\right| \leq 1 \forall i, j\right\} \\
& =\sup \left\{-\langle\operatorname{div} \xi, u\rangle_{X}:\left|\xi_{i, j}\right| \leq 1 \forall i, j\right\} \\
& =\sup _{p}\langle p, x\rangle_{X}-H(p)
\end{aligned}
$$

where, letting

$$
K=\left\{p=-\operatorname{div} \xi \in X:\left\|\xi_{i, j}\right\| \leq 1 \forall i, j\right\},
$$

we have $H(p)=0$ for $p \in K$ and $H(p)=+\infty$ if $p \notin K$ ( $H$ is called the "characteristic function of $\left.K^{\prime \prime}\right)$. Hence $J$ is the Legendre-Fenchel conjugate $H^{*}$ of this function $H$.

Since $K$ is closed and convex, $H \in \Gamma^{0}$, so that $J^{*}=H^{* *}=H$. Hence the dual problem (44) is also

$$
\min _{v \in K} \frac{1}{2}\left\|v-\frac{g}{\lambda}\right\|^{2}=\min _{|\xi|_{i, j} \leq 1} \frac{1}{2}\left\|\operatorname{div} \xi+\frac{g}{\lambda}\right\|^{2}
$$

and we recover $u$ by letting $u=g-\lambda v=g+\lambda \operatorname{div} \xi$.

We will see that this problem has a structure which makes it nicer (easier) to solve than the primal problem (38). 


\subsection{4 "Proximal" operator}

We end this section by introducing more generally the "proximal" operator associated to a function $F \in \Gamma^{0}$. For any $F \in \Gamma^{0}$ it is not hard to show that for any $\delta>0$, problem

$$
\min _{y} \delta F(y)+\frac{1}{2}\|y-x\|^{2}
$$

always have a solution, which is unique. The equation for this solution $y$ is

$$
\delta \partial F(y)+y-x \ni 0
$$

hence

$$
y=(I+\delta \partial F)^{-1}(x)
$$

is well-defined and uniquely defined. The mapping $(I+\delta \partial F)^{-1}$ is called the "proximal map" of $\delta F$ and sometimes denoted $\operatorname{prox}_{\delta F}$. The following identity, which is exactly our derivation of the dual problem in the previous section, is due to Moreau:

$$
x=(I+\delta \partial F)^{-1}(x)+\delta\left(I+\frac{1}{\delta} \partial F^{*}\right)^{-1}\left(\frac{x}{\delta}\right),
$$

and for $\delta=1$ it reduces to:

$$
x=(I+\partial F)^{-1}(x)+\left(I+\partial F^{*}\right)^{-1}(x) .
$$

Examples If $F(x)=\alpha x^{2} / 2$ for some $\alpha>0$, we check that

$$
(I+\delta \partial F)^{-1}(x)=\frac{x}{1+\delta \alpha} .
$$

The reader may check that this is coherent with (48) and the fact that $F^{*}(p)=$ $p^{2} /(2 \alpha)$.

If $F(x)$ is the characteristic function of a closed, convex set $C \subset X$, that is $F(x)=0$ if $x \in C$ and $+\infty$ else, then

$$
(I+\delta \partial F)^{-1}(x)=\Pi_{C}(x),
$$

the Euclidean projection on $C$ of $x$, which actually minimizes $\|x-y\|^{2}$ over all $y \in C$. On the other hand, it follows from (48) that

$$
\bar{y}=\left(I+\frac{1}{\delta} \partial F^{*}\right)^{-1}(y)=y-\frac{1}{\delta} \Pi_{C}(\delta y)
$$


which is some kind of "shrinkage" or "soft-thresholding" of $y$ from which one removes the projection on $(1 / \delta) C$. The point $\bar{y}$ is the minimizer of

$$
\min _{z} \frac{\|z-y\|^{2}}{2}+\frac{1}{\delta} h_{C}(z)
$$

where $F^{*}=h_{C}$ is the support function of $C$, defined by $h_{C}(z)=\sup _{x \in C}\langle z, x\rangle$.

In the sequel we introduce a few possible algorithms to solve (38) or (46).

\subsection{Gradient descent}

Consider first an elementary problem which is to minimize over $X$ a function $F \in$ $\Gamma^{0}$, which is differentiable and such that $\nabla F$ is Lipschitz with some constant $L$ (one says that $F$ is $C^{1,1}$ ).

It is not the case for (38), but it is the case for the approximation

$$
F_{\varepsilon}(u)=\sum_{i, j} \sqrt{\varepsilon^{2}+\left|(\nabla u)_{i, j}\right|^{2}}+\frac{1}{2}\|u-g\|^{2}
$$

for any $\varepsilon>0$ (and it is clear that as $\varepsilon \rightarrow 0$, this problem will approximate the other one). In this case, $\nabla F_{\varepsilon}$ is Lipschitz with a constant of order $1 / \varepsilon$.

Then, the most straigthforward approach is the "gradient descent": choose $h>0$ a step, any $x^{0} \in X$ and let for any $n \geq 0$

$$
u^{n+1}=u^{n}-h \nabla F\left(u^{n}\right) .
$$

(of course the step $h$ needs not, and should not, be constant, but for simplicity we stick to this case).

This method is not very efficient (and should not be used!). A complexity bound can be derived:

Theorem 10 (Nesterov [59]). Assume $h \in(0,2 / L)$ : then $F\left(u^{k}\right) \rightarrow \min F=F\left(x^{*}\right)$ as $k \rightarrow \infty$. The best rate of convergence is obtained for $h=1 / L$, and is

$$
F\left(u^{k}\right)-F\left(x^{*}\right) \leq \frac{2 L\left\|u^{0}-u^{*}\right\|^{2}}{k+4}
$$

Observe that the estimate depends on the quality of the initial guess. For a proof, see [59], Thm 2.1.14 and Cor. 2.1.2.

For solving the approximation $F_{\varepsilon}$, one sees that the step $h$ should be taken of order $\varepsilon$. This approach cannot be used to solve the dual problem (46): indeed, 
although the objective function is quite smooth in this case (and the gradient, $\nabla(\operatorname{div} p+g / \lambda)$, is Lipschitz with constant $L \leq 8)$, it has to be minimized on a convex set (hence with a constraint). For this reason, we need to introduce constrained variants of the Gradient Descent algorithm.

\subsubsection{Splitting, and Projected gradient descent}

We follow in this presentation the paper of Beck and Teboulle [13]. Assume we want to solve

$$
\min _{x \in X} F(x)+G(x)
$$

where: $F$ is $C^{1,1}$ ( $\nabla F$ is $L$-Lipschitz), and $G$ is "simple", meaning that the "prox" $(I+h \partial G)^{-1}$ is easy to compute. This is for instance the case for the dual problem (46): in this case

$$
F(p)=\frac{1}{2}\|\operatorname{div} p+g\|^{2} \text { and } G(p)= \begin{cases}0 & \text { if }\left\|p_{i, j}\right\| \leq 1 \forall i, j \\ +\infty & \text { else. }\end{cases}
$$

We see that (see section 3.2.4)

$$
(I+h \partial G)^{-1}(p)=\arg \min _{q} \frac{1}{2}\|q-p\|^{2}+h G(q)=\Pi_{C}(p)
$$

where $\Pi_{C}$ denotes the orthogonal projection onto $\left\{p:\left\|p_{i, j}\right\| \leq 1 \forall i, j\right\}$ and is straightforward to compute.

This is an example of "splitting" (introduced first by Douglas and Rachford, see for instance [48] for a general form): one solves successively one step of the gradient descent of $F$ (in an explicit way), and one step of the gradient descent of $G$ (in an implicit way), in order to obtain a "full" gradient descent of $F+G$. Hence the term "forward-backwards" splitting, see [27].

In case $G$ is, like here, a characteristic function and $(I+h \partial G)^{-1}$ is a projection), then it reduces to a "projected gradient algorithm": one does one explicit step of descent in $F$, and then reproject the point on our constraint.

The resulting algorithm is hence as follows: we choose $x^{0} \in X$, and let

$$
x^{n+1}=(I+h \partial G)^{-1}\left(x^{n}-h \nabla F\left(x^{n}\right)\right)
$$

for a given, fixed step $h>0$. One can also write the iteration $x^{n+1} \in x^{n}-$ $h\left(\nabla F\left(x^{n}\right)+\partial G\left(x^{n+1}\right)\right)$ which makes apparent the forward-backwards splitting. 
Again, this algorithm is quite slow, but it is interesting to understand the intuitive idea behind it. In fact, if $\nabla F$ is $L$-Lipschitz, we can write for any $x, y \in X$

$$
\begin{aligned}
F(y)=F(x)+\left\langle\int_{0}^{1} \nabla F(x+t\right. & (y-x)) d t, y-x\rangle \\
& \leq F(x)+\langle\nabla F(x), y-x\rangle+\frac{L}{2}\|y-x\|^{2}
\end{aligned}
$$

so that the parabola $y \mapsto Q_{L}(y, x)=F(x)+\langle\nabla F(x), y-x\rangle+\frac{L}{2}\|y-x\|^{2}$ approximates from above the function $F$. Now, assume $x=x^{n}$ and we replace the minimization of $F$, at step $n$, with the minimization of $Q_{L}\left(y, x^{n}\right)$ w.r. $y$. Then, we find $y=x^{n}-(1 / L) \nabla F\left(x^{n}\right)$, that is, a step of the gradient descent algorithms with step $1 / L$. This is a way to interpret that algorithm, and provides a natural way to extend it to the minimization of $F+G$ : indeed, we can now let

$$
Q_{L}(y, x)=F(x)+\langle\nabla F(x), y-x\rangle+\frac{L}{2}\|y-x\|^{2}+G(y)
$$

and we see, as before, that $F(y)+G(y) \leq Q_{L}(y, x)$ for any $x, y$.

Now, consider the problem $\min _{y} Q_{L}\left(y, x^{n}\right)$. The equation is

$$
\nabla F\left(x^{n}\right)+L\left(y-x^{n}\right)+\partial G(y) \ni 0
$$

and we find that the solution is nothing else than the iterate $x^{n+1}$ given by (49), provided $h=1 / L$.

The following is Lemma 2.3 in [13]

Lemma 3.8. Let $x \in X$, and $h>0$, and let $y=\arg \min Q_{1 / h}(\cdot, x)$ be such that $F(y)+G(y) \leq Q_{1 / h}(y, x)$ (which is true as soon as $h<1 / L$, by (50)). Then for any $z \in X$

$$
\begin{aligned}
& (F(z)+G(z))-(F(y)+G(y)) \\
& \quad \geq \frac{1}{2 h}\|x-y\|^{2}+\frac{1}{h}\langle x-z, y-x\rangle=\frac{1}{2 h}\left(\|y-z\|^{2}-\|x-z\|^{2}\right) .
\end{aligned}
$$

Proof. By assumption,

$$
(F(z)+G(z))-(F(y)+G(y)) \geq F(z)+G(z)-Q_{1 / h}(y, x) .
$$

Also, $F(z) \geq F(x)+\langle\nabla F(x), z-x\rangle$, while $G(z) \geq G(y)+\langle p, z-y\rangle$ where $p=$ $(x-h \nabla F(x)-y) / h \in \partial G(y)$. Hence

$$
F(z)+G(z) \geq F(x)+G(y)+\langle\nabla F(x), z-x\rangle+\langle p, z-y\rangle .
$$


We deduce from (52) that

$$
\begin{gathered}
(F(z)+G(z))-(F(y)+G(y)) \\
\geq F(x)+G(y)+\langle\nabla F(x), z-x\rangle+\langle p, z-y\rangle \\
-F(x)-\langle\nabla F(x), y-x\rangle-\frac{1}{2 h}\|y-x\|^{2}-G(y) \\
=\langle\nabla F(x)+p, z-y\rangle-\frac{1}{2 h}\|y-x\|^{2} \\
=\frac{1}{h}\langle x-y, z-y\rangle-\frac{1}{2 h}\|y-x\|^{2}=\frac{1}{2 h}\|y-x\|^{2}+\frac{1}{h}\langle x-y, z-x\rangle .
\end{gathered}
$$

This allows to prove the following result (see Beck and Teboulle [13], Thm 3.1):

Theorem 11. Let $\left(x^{n}\right)_{n}$ satisfy (49), and $h=1 /$ L. Then

$$
\left(F\left(x^{k}\right)+G\left(x^{k}\right)\right)-\left(F\left(x^{*}\right)+G\left(x^{*}\right)\right) \leq \frac{L\left\|x^{0}-x^{*}\right\|^{2}}{2 k}
$$

for any $k \geq 1$, and for any solution $x^{*}$ of the problem.

Hence the rate of convergence is essentially the same as the gradient descent's.

Proof. We follow the very elegant proof of Beck and Teboulle [13]. First use (51) with $z=x^{*}, x=x^{n}, h=1 / L$ :

$$
\frac{2}{L}\left(\left(F\left(x^{*}\right)+G\left(x^{*}\right)\right)-\left(F\left(x^{n+1}\right)+G\left(x^{n+1}\right)\right)\right) \geq\left\|x^{n+1}-x^{*}\right\|^{2}-\left\|x^{n}-x^{*}\right\|^{2},
$$

which we sum from $n=0$ to $k-1$ :

$$
\frac{2}{L}\left(k\left(F\left(x^{*}\right)+G\left(x^{*}\right)\right)-\sum_{n=1}^{k}\left(F\left(x^{n}\right)+G\left(x^{n}\right)\right)\right) \geq\left\|x^{k}-x^{*}\right\|^{2}-\left\|x^{0}-x^{*}\right\|^{2} .
$$

We use (51) again with $z=x=x^{n}, h=1 / L$ :

$$
\frac{2}{L}\left(\left(F\left(x^{n}\right)+G\left(x^{n}\right)\right)-\left(F\left(x^{n+1}\right)+G\left(x^{n+1}\right)\right)\right) \geq\left\|x^{n+1}-x^{n}\right\|^{2},
$$

which we multiply by $n$ before summing from 0 to $k-1$ :

$$
\begin{aligned}
& \frac{2}{L}\left(\sum_{n=0}^{k-1} n\left(F\left(x^{n}\right)+G\left(x^{n}\right)\right)-\sum_{n=1}^{k}(n-1)\left(F\left(x^{n}\right)+G\left(x^{n}\right)\right)\right)= \\
& \quad \frac{2}{L}\left(\sum_{n=1}^{k-1}\left(F\left(x^{n}\right)+G\left(x^{n}\right)\right)-(k-1)\left(F\left(x^{k}\right)+G\left(x^{k}\right)\right)\right) \geq \sum_{n=0}^{k-1} n\left\|x^{n+1}-x^{n}\right\|^{2} .
\end{aligned}
$$


We add this last equation to (54) and find:

$$
\begin{aligned}
\frac{2}{L}\left(k \left(F\left(x^{*}\right)\right.\right. & \left.\left.+G\left(x^{*}\right)\right)-k\left(F\left(x^{k}\right)+G\left(x^{k}\right)\right)\right) \\
& \geq\left\|x^{k}-x^{*}\right\|^{2}-\left\|x^{0}-x^{*}\right\|^{2}+\sum_{n=0}^{k-1} n\left\|x^{n+1}-x^{n}\right\|^{2} \geq-\left\|x^{0}-x^{*}\right\|^{2}
\end{aligned}
$$

from which (53) follows.

Hence: this provides a convergent (but slow) way to minimize the dual problem (and many variants).

\subsubsection{Improvements: optimal first-order methods}

The rate of convergence of theses methods are slow. It is shown by Nesterov [59] that first order method can theoretically not achieve a better rate of convergence than $C / k^{2}$ (after $k$ iterations). A few variants of the previous methods achieve such a rate of convergence and are recommended in the implementations.

Nesterov/Beck and Teboulle's acceleration In [13] the following iteration is proposed, as an variant of an acceleration for the gradient descent proposed by Nesterov in [58]: let $x^{0} \in X=\mathbb{R}^{N}, y^{1}=x^{0}, t_{1}=1$, and:

$$
\begin{gathered}
x^{k}=\left(I+\frac{1}{L} \partial G\right)^{-1}\left(y^{k}-\frac{1}{L} \nabla F\left(y^{k}\right)\right), \\
t_{k+1}=\frac{1+\sqrt{1+4 t_{k}^{2}}}{2}, \quad y^{k+1}=x^{k}+\frac{t_{k}-1}{t_{k+1}}\left(x^{k}-x^{k-1}\right) .
\end{gathered}
$$

Then:

Theorem 12 (Beck and Teboulle [13, Thm. 4.1]). For any minimizer $x^{*}$,

$$
F\left(x^{k}\right)+G\left(x^{k}\right)-\left(F\left(x^{*}\right)+G\left(x^{*}\right)\right) \leq \frac{2 L\left\|x^{0}-x^{*}\right\|^{2}}{(k+1)^{2}} .
$$

Yu. Nesterov himself also proposed in improvement of his earlier algorithm for nonsmooth problems in [60], which is similar in spirit but a bit more complex to describe. See also [15] for similar accelerations for a smaller class of problems. 


\subsection{Augmented Lagrangian approaches}

Another class of methods for solving (38) are the "augmented Lagrangian" methods, also known as "split Bregman" iterations, or "Alternating directions method of multipliers"

The basic idea is as follows: instead of solving (38), we solve the constrained problem

$$
\min _{p=\nabla u} \lambda\|p\|_{2,1}+\frac{1}{2}\|u-g\|^{2} .
$$

Then, to enforce the constraint, we use an augmented Lagrangian approach, which consists in introducing

$$
L_{\alpha}(p, u, \mu)=\|p\|_{2,1}+\frac{1}{2}\|u-g\|^{2}+\langle\mu, p-\nabla u\rangle+\frac{\alpha}{2}\|p-\nabla u\|^{2}
$$

where here, $\mu \in Y$ is a Lagrange multiplier for the constraint $p=\nabla u$. The method consists then in minimizing alternatively $L$ w.r. $p, u$, and maximizing w.r. $\mu$ :

$$
\begin{aligned}
& u^{k+1}=\arg \min _{u} L\left(p^{k}, u, \mu^{k}\right) \\
& p^{k+1}=\arg \min _{p} L\left(p, u^{k+1}, \mu^{k}\right) \\
& \mu^{k+1}=\mu^{k}+\alpha\left(p^{k+1}-\nabla u^{k+1}\right)
\end{aligned}
$$

and this method is shown to converge. It seems it was first studied by Gabay and Mercier, 1976, Glowinski and Marrocco, 1975. Then, it was analyzed in a more general framework in [30]. See also [32] and the references therein for a recent study on these approaches.

\subsection{Primal-dual approaches}

The last interesting family of algorithms to solve (38) are maybe the primal-dual approaches, or "Arrow-Hurwicz" type methods. The idea goes back to [11], but seems to be first found in this framework in a paper of Appleton and Talbot [10]. Consider the formulation (39), using Legendre-Fenchel's duality, it can also be written as:

$$
\min _{x \in X} \max _{y \in Y}\langle y, A x\rangle-F^{*}(y)+G(x)
$$

and the idea is to alternate gradient descent in $x$ and ascent in $y$. Take care that in some cases, it provides iterations which are almost identical to the iterations provided by the previous splitting approaches. An important observation is that standard convex analysis shows that under very weak assumptions, the min and 
max may be swapped in (55). This is another approach to the dual problem of (39), which can be found by writing

$$
\begin{aligned}
& \min _{x \in X} F(A x)+G(x)=\min _{x \in X} \max _{y \in Y}\langle y, A x\rangle-F^{*}(y)+G(x) \\
& =\max _{y \in Y} \min _{x \in X}\left\langle A^{*} y, x\right\rangle+G(x)-F^{*}(y)=\max _{y \in Y}-\left(G^{*}\left(-A^{*} y\right)-F^{*}(y)\right) .
\end{aligned}
$$

Moreover, one deduces immediately that the quantity

$$
\mathcal{G}(x, y)=F(A x)+G(x)+G^{*}\left(-A^{*} y\right)-F^{*}(y),
$$

known as the primal-dual gap, is always nonnegative, and vanishes only if $(\hat{x}, \hat{y})$ is a saddle-point of (55), hence satisfying

$$
\langle y, A \hat{x}\rangle-F^{*}(y)+G(\hat{x}) \leq\langle\hat{y}, A \hat{x}\rangle-F^{*}(\hat{y})+G(\hat{x}) \leq\langle\hat{y}, A x\rangle-F^{*}(\hat{y})+G(x)
$$

for all $(x, y) \in X \times Y$.

This suggests the following approach, which consists in performing simultaneously an approximate gradient descent in $x$ and gradient ascent in $y$ : choose $x^{0}, y^{0}$, $\tau, \sigma>0$ two time-steps, and let

$$
\begin{aligned}
& y^{n+1}=\left(I+\sigma \partial F^{*}\right)^{-1}\left(y^{n}+\sigma A x^{n}\right) \\
& x^{n+1}=(I+\tau \partial G)^{-1}\left(x^{n}-\tau A^{*} y^{n+1}\right)
\end{aligned}
$$

The scheme, as is, is proposed in a paper of Zhu and Chan [73], with an interesting (and very efficient) acceleration which is obtained by varying the time-steps, but unfortunately no proof of convergence exists. A global study of such schemes is found in a recent preprint [33].

We have provided recently in [63] the following variant, inspired by a paper of L. Popov [65]. The algorithm is as follows: we choose $x^{0}=\bar{x}^{0}, y^{0}$, and let for each $n \geq 0$ :

$$
\begin{aligned}
& y^{n+1}=\left(I+\sigma \partial F^{*}\right)^{-1}\left(y^{n}+\sigma A \bar{x}^{n}\right) \\
& x^{n+1}=(I+\tau \partial G)^{-1}\left(x^{n}-\tau A^{*} y^{n+1}\right) \\
& \bar{x}^{n+1}=2 x^{n+1}-x^{n}
\end{aligned}
$$

(Observe that the algorithm could also be written with a variable $\bar{y}$ instead of $\bar{x}$, and iterating first in $x$, then in $y$.) This is a slight variant of "extragradient" algorithms (first proposed by G. Korpelevich [44]) and which is know to converge.

This algorithm has been recently presented as a particular case of a more general algorithm in [33], and a proof of convergence is also given there. It also reduces 
trivially to the standard Douglas-Rachford splitting in case $A=I d$ (and probably if $A$ is invertible), just perform the change of variable $v^{n}=y^{n}-x^{n} / \tau$ and check against the formula proposed in [48, eq. (10)]. We give here an alternate proof of convergence, which is inspired from [65] and [57]. Actually, we can show convergence of the iterates to a solution in finite dimension, while in the general case, mimicking the proof of [57] where A. Nemirovski computes rates of convergence for a general version of the extragradient algorithm, we find a convergence of a primal-dual gap to zero, in $O(1 / n)$. For practical use, we introduce the partial primal-dual gap

$\mathcal{G}_{B_{1} \times B_{2}}(x, y)=\max _{y^{\prime} \in B_{2}}\left\langle y^{\prime}, A x\right\rangle-F^{*}\left(y^{\prime}\right)+G(x)-\min _{x^{\prime} \in B_{1}}\left\langle y, A x^{\prime}\right\rangle-F^{*}(y)+G\left(x^{\prime}\right)$, $\left(\mathcal{G}=\mathcal{G}_{X \times Y}\right)$. Then, as soon as $B_{1} \times B_{2}$ contains a saddle-point $(\hat{x}, \hat{y})$, defined by (56), we have

$$
\mathcal{G}_{B_{1} \times B_{2}}(x, y) \geq\langle\hat{y}, A x\rangle-F^{*}(\hat{y})+G(x)-\langle y, A \hat{x}\rangle-F^{*}(y)+G(\hat{x}) \geq 0
$$

and it vanishes only if $(x, y)$ is itself a saddle-point.

Theorem 13. Let $L=\|A\|$ and assume problem (55) has a saddlepoint $(\hat{x}, \hat{y})$. Then, if $\tau \sigma L^{2}<1$ and $\left(x_{n}, \bar{x}_{n}, y_{n}\right)$ are defined by (57):

(a) For any $n$,

$$
\frac{\left\|y^{n}-\hat{y}\right\|^{2}}{2 \sigma}+\frac{\left\|x^{n}-\hat{x}\right\|^{2}}{2 \tau} \leq C\left(\frac{\left\|y^{0}-\hat{y}\right\|^{2}}{2 \sigma}+\frac{\left\|x^{0}-\hat{x}\right\|^{2}}{2 \tau}\right)
$$

where the constant $C \leq\left(1-\tau \sigma L^{2}\right)^{-1}$;

(b) If we let $x_{N}=\left(\sum_{=1}^{N} x^{n}\right) / N$ and $y_{N}=\left(\sum_{n=1}^{N} y^{n}\right) / N$, for any bounded $B_{1} \times$ $B_{2} \subset X \times Y$ the restricted gap has the following bound:

$$
\mathcal{G}_{B_{1} \times B_{2}}\left(x_{N}, y_{N}\right) \leq \frac{C\left(B_{1}, B_{2}\right)}{n} .
$$

Moreover, the weak cluster points of $\left(x_{N}, y_{N}\right)$ are saddle-points of (55);

(c) If the dimension of the spaces $X$ and $Y$ is finite, then there exists a saddlepoint $\left(x^{*}, y^{*}\right)$ such that $x^{n} \rightarrow x^{*}$ and $y^{n} \rightarrow y^{*}$ (of course, then, also $\left(x_{n}, y_{n}\right) \rightarrow$ $\left.\left(x^{*}, y^{*}\right)\right)$.

The proof of Theorem 13 is found in Appendix A. 
The estimate (59) is relatively weak but seems to show that the method is in some sense optimal (but slow). However, we'll see in the next section 3.7 that the convergence can be apparently improved by varying the time-steps and relaxation parameters, as suggested in [73], although we do not have a clear explanation for this (and it is possible that this acceleration is problem-dependent, as opposed to the result of Theorem 12).

Observe that if $F^{*}(y) /|y| \rightarrow \infty$ as $|y| \rightarrow \infty$, then for any $R>0, F^{*}(y) \geq R|y|$ for $y$ large enough which yields that $\operatorname{dom} F \subset B(0, R)$. Hence $F$ has full domain. It is classical that in this case, $F$ is locally Lipschitz in $Y$.

One checks, then, that

$$
\max _{y \in Y}\left\langle y, A x_{n}\right\rangle-F^{*}(y)+G\left(x_{n}\right)=F\left(A x_{n}\right)+G\left(x_{n}\right)
$$

is reached at some $y \in \partial F\left(A x_{n}\right)$, which is globally bounded thanks to (58). It follows from (59) that $F\left(A x_{n}\right)+G\left(x_{n}\right)-(F(A \bar{x})+G(\bar{x})) \leq C / n$ for some constant depending on the starting point $\left(x^{0}, y^{0}\right), F$ and $L$. In the same way, if $\lim _{|x| \rightarrow \infty} G(x) /|x| \rightarrow \infty$, we have $F^{*}\left(y_{n}\right)+G^{*}\left(-A^{*} y_{n}\right)-\left(F^{*}(\hat{y})+G^{*}\left(-A^{*} \hat{y}\right)\right) \leq C / n$. If both $F^{*}(y) /|y|$ and $G(x) /|x|$ diverge as $|y|$ and $|x|$ go to infinity, then the global gap $\mathcal{G}\left(x_{n}, y_{n}\right) \leq C / n$.

It is easy to check that this approach is useful for many variants of (38) or similar discretizations of (16). We leave this as an exercise to the reader, see also the examples in section 3.7 .

\subsection{Graph-cut techniques}

A last approach to solve (38) (or, in fact, a slight variant) is to use "graph-cuts" or "maximal flow" algorithms [1]. It has been noticed long ago [62] that maximal flow/minimum cut techniques could be used to solve discrete problems of the form (16), that is, to compute finite sets minimizing a discrete variant of the perimeter and an additional external field term.

This approach has gained in popularity in the past ten years, mostly because of incredibly fast algorithms, specially coined for image processing applications, see in particular [16].

Combined with the discrete counterpart of Proposition 2.6, it leads to efficient techniques for solving (only) the denoising problem $(R O F)$ in the discrete setting. 
In fact, although this approach is strictly limited to problems of the form

$$
\min _{u \in \mathbb{R}^{N \times N}} J(u)+\sum_{i, j} \Psi_{i, j}\left(u_{i, j}\right)
$$

with each function $\Psi_{i, j}$ convex, and $J$ involving pairwise interactions, ${ }^{3}$ such as

$$
J(u)=\sum_{i, j}\left|u_{i+1, j}-u_{i, j}\right|+\left|u_{i, j+1}-u_{i, j}\right|,
$$

an algorithm described in a paper by D. Hochbaum [41] provides a way to solve exactly (up to machine precision) this minimization for simple terms $\Psi_{i, j}\left(u_{i, j}\right)$ such as $\left(u_{i, j}-g_{i, j}\right)^{2}$.

We will not describe this approach in these notes, and refer to [23], and the references therein, for details.

\subsection{Comparisons of the numerical algorithms}

It is of course naive to believe that one single algorithm will fulfill the needs of all inverse problems which can be regularized by the total variation. However, the method (57) has the advantage of being easy to adapt to many different situations, and provide good results in most cases. Clearly, research should then focus on improving the numerical methods specifically for each particular applications, once it has been checked that the approach was efficient. In the following, we will present a comparison of the algorithms discussed in the previous sections for computing the minimizer of the discrete $(R O F)$ problem (38). Besides this, we will also compare different discrete approximations of the total variation including anisotropic approximations and upwind schemes.

In order to evaluate the performance of each algorithm, we used the following procedure. First, we ran the proposed modified extragradient method (57) for a large number of iterations (10000) to generate the ground truth solution (the primal dual gap was always less then $10^{-6}$ ). Then, we ran the different algorithms until the root mean squared error (RMSE) of the current iterate to the ground truth solution was less than $t o l=10^{-3}$.

Table 1 gives an overview of the algorithms and parameter settings we used in our performance evaluation. All algorithms were implemented in pure Matlab, except for the graph-cut algorithm, whose main routine was implemented in optimized $\mathrm{C} / \mathrm{C}++$ (see [23] for more details). In addition, we implemented an parallel

\footnotetext{
${ }^{3}$ In fact, slightly more complex situations can be considered, see for instance [43].
} 


\begin{tabular}{|l||l|}
\hline P-GD & Primal, gradient descend, $\varepsilon=0.001$ \\
\hline D-PGD & Dual, projected gradient descend \\
\hline D-BT & Dual, fast iterative shrinkage thresholding algorithm $[13]$ \\
\hline PD-AL & Primal-dual, augmented Lagrangian approach $[32], \alpha=20$ \\
\hline PD-ME & Primal-dual, modified extragradient, $\tau=0.01$ \\
\hline PD-MEG & Primal-dual, modified extragradient, GPU version, $\tau=0.01$ \\
\hline PD-ZC & Primal-dual, Arrow-Hurwitz method, varying steps [73] \\
\hline GC-8 $(16)$ & Graph-cut, $8(16)$-connected graph, 8-bit accuracy $[23]$ \\
\hline
\end{tabular}

Table 1: Explanation of the algorithms of the performance evaluation.

version of the modified extragradient algorithm on the (graphics processing unit) GPU using the CUDA framework. Executed on a Nvidia Tesla C1060 GPU, this results in a dramatic speedup of approximately 200 compared to the pure Matlab implementation. Note that on the other hand, an efficient parallel implementation of graph-cut algorithms is still an open problem. It can therefore be expected that algorithms that can be executed in parallel will play an important role in future. Finally, we note that since graph-cut algorithms compute the exact solution with respect to a discretized (8 Bit) solution space, their results are not directly compareable to those of the continuous optimization algorithms.

Figure 7 shows the input image and results of our evaluation. The first row shows the clean and noisy input images and the second row shows results for different values of the regularization parameter $\lambda$. In the last row we provide a comparison between the graph-cut algorithms and the continuous minimization algorithms for $\lambda=1$. The graph cut algorithm was executed using a 8- and a 16-connected graph. The modified extragradient method was executed using the simple forward differences approximation (see (37)) and a more sophisticated upwind scheme proposed in [24]. One can see that for a 8-connected graph, the so-called metrication errors of graph-cut algorithms are clearly visible. For a 16-connected graph, the results are very close to those of the continuous algorithms. Comparing the results of the continuous algorithms, one can observe that the upwind scheme delivers sharp edges almost independend of the edge orientation, whereas the simple forward differences approximation exhibits some blurring at certain edge orientations.

Table 2 shows the results of our performance evaluation. The table entries refer to the number of iterations the algorithm needed until the RMSE was less 


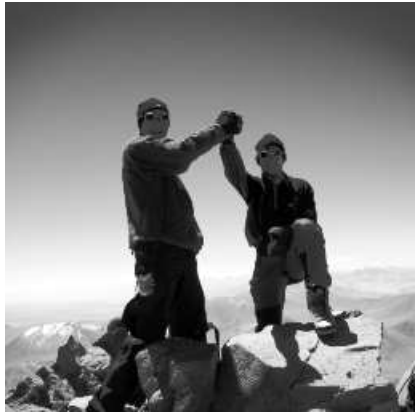

(a) Clean image

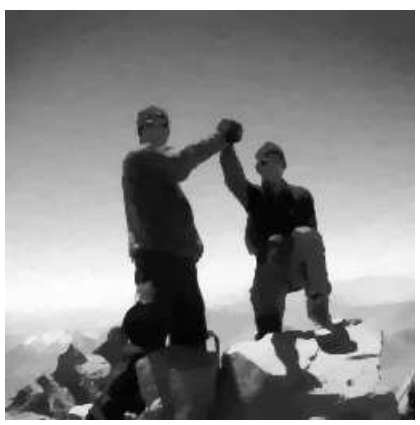

(c) $\lambda=1 / 16$

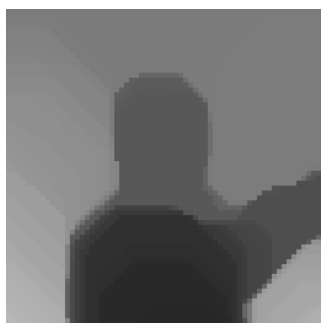

(f) GC-8

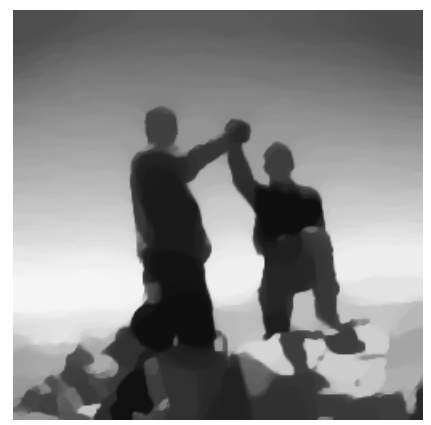

(d) $\lambda=1 / 4$

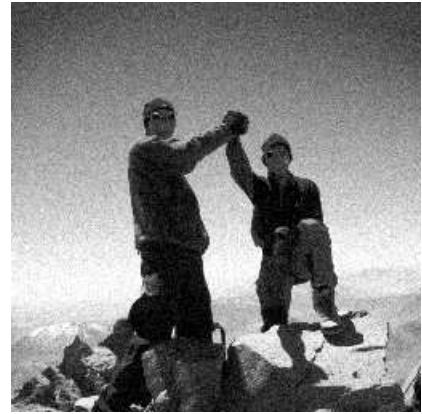

(b) Noisy image $(\sigma=0.05)$

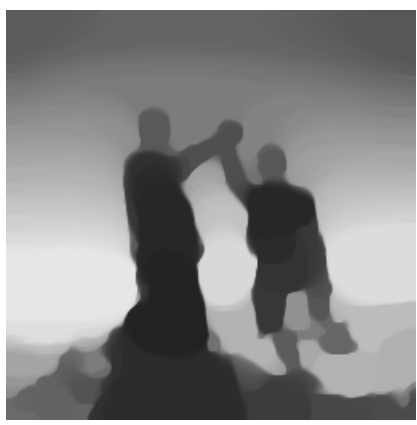

(e) $\lambda=1$

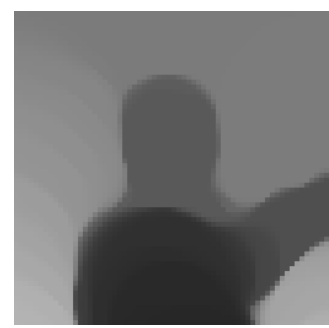

(i) PD-ME, upwind

Figure 7: Results and comparisons of the proposed minimization algorithms. (a) and (b) show the clean input image of size $(256 \times 256)$ and a degraded version generated by adding white Gaussian noise of standard deviation $\sigma=0.05$. (c)-(e) show some results of the minimization algorithms for different values of the regularization parameter $\lambda$. (f)-(i) show a comparison of different discrete approximations of the total variation. (f) and (g) are anisotropic polygonial approximations of different order used by the graph cut techniques. (h) and (i) are more isotropic approximations used by the primal-dual minimization algorithm. 


\begin{tabular}{|l||c|c|c|c|c|}
\hline$\lambda$ & $1 / 16$ & $1 / 8$ & $1 / 4$ & $1 / 2$ & 1 \\
\hline \hline P-GD & $800 / 20.6$ & $-/-$ & $-/-$ & $-/-$ & $-/-$ \\
\hline D-PGD & $110 / 2.57$ & $350 / 8.93$ & $1120 / 27.93$ & $3340 / 86.78$ & $-/-$ \\
\hline D-BT & $40 / 1.28$ & $80 / 2.54$ & $140 / 4.72$ & $270 / 8.31$ & $460 / 15.41$ \\
\hline PD-AL & $50 / 1.40$ & $90 / 2.65$ & $150 / 4.55$ & $250 / 7.55$ & $420 / 12.5$ \\
\hline PD-ME & $40 / 1.09$ & $70 / 2.06$ & $120 / 3.64$ & $220 / 6.04$ & $410 / 10.99$ \\
\hline PD-MEG & $40 / 0.005$ & $70 / 0.009$ & $120 / 0.016$ & $220 / 0.029$ & $410 / 0.053$ \\
\hline PD-ZC & $20 / 0.56$ & $50 / 1.30$ & $90 / 2.43$ & $150 / 3.84$ & $300 / 8.02$ \\
\hline GC-8 & $-/ 0.59$ & $-/ 0.67$ & $-/ 0.79$ & $-/ 0.95$ & $-/ 1.31$ \\
\hline GC-16 & $-/ 1.10$ & $-/ 1.27$ & $-/ 1.58$ & $-/ 2.06$ & $-/ 2.96$ \\
\hline
\end{tabular}

Table 2: Performance evaluation of various minimization algorithms. The entries in the table refer to [iterations/time (sec)].

than tol. The second numbers refer to the respective execution time in seconds. If no entry is present, the algorithm did not meet the convergence criterion within 10000 iterations. At first, one can observe that for larger values of $\lambda$ the problem gets harder for all algorithms. Actually, simple gradient based methods even fail to converge within 10000 iterations for larger values of $\lambda$. The PD-ZC algorithm is the fastest iterative method. It is slightly better than the proposed PD-ME algorithm. However, while the PD-ME method is proven to converge with a certain rate, a proof of convergence for the PD-ZC method (if there is one) is still an open problem. Furthermore, while the PD-ZC method is tailored for minimizing the $(R O F)$ model, we will see in the next sections that the PD-ME method is applicable for a much larger class of problems. Interestingly, the PD-AL algorithm, which is often considered to be the most competing algorithm for minimizing the $(R O F)$ model, is clearly outperformed by PD-ZC and PD-ME. The graph-cut algorithms are fast and deliver exact discrete solutions but an efficient parallelization is still an open problem. In contrast, the continuous PD-ME algorithm is easy to parallelize and its GPU-based variant yields the overall fastest method. 


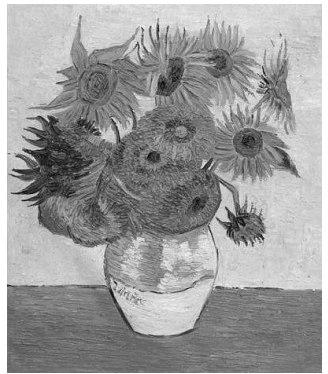

(a) Original image

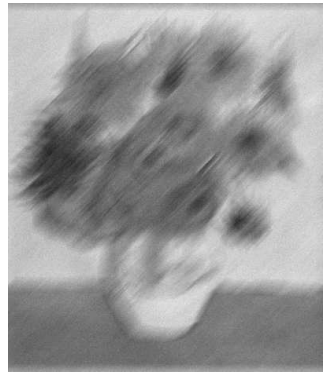

(b) Degraded image

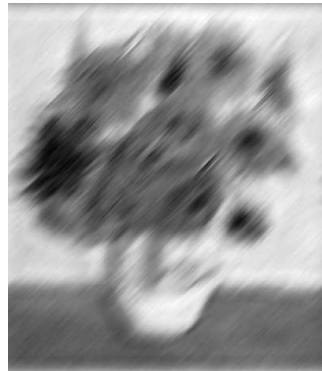

(c) Wiener filter

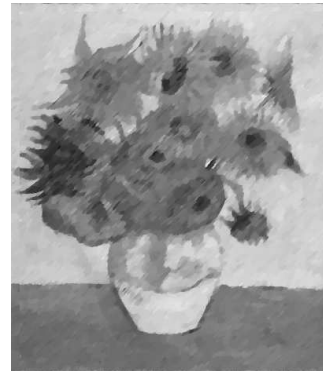

(d) TV-deblurring

Figure 8: Motion deblurring using total variation regularization. (a) and (b) show the clean image and a degraded version containing motion blur of approximately 30 pixels and Gaussian noise of standard deviation $\sigma=0.02$. (c) is the result of standard Wiener filtering. (d) is the result of the total variation based deblurring method. Note that the TV-based method yields visually much more appealing results.

\section{Applications}

\subsection{Total variation based image deblurring and zooming}

The standard $(R O F)$ model can be easily extended for image deblurring and digital zooming.

$$
\min _{u}\left\{\int_{\Omega}|D u|+\frac{\lambda}{2} \int_{\Omega}(A u-f)^{2} d x\right\}
$$

where $\Omega \subset \mathbb{R}^{2}$ is the domain of the image and $A$ is a linear operator. In the case of image deblurring, $A$ is the blurring kernel. In the case of image zooming, $A$ describes the downsampling procedure, which is often assumed to be a blurring kernel followed by subsampling operator. This problem can be easily rewritten in terms of a saddle-point problem (55).

$$
\min _{u} \max _{p, q}\langle p, D u\rangle+\langle q, A u-f\rangle-I_{\|p\|_{\infty} \leq 1}-\frac{1}{2 \lambda}\|q\|^{2},
$$

which can then be solved by the iterates (57).

Figure 8 shows the application of the energy (60) to motion deblurring. While the classical Wiener filter is not able to restore the image the total variation based approach yields a far better result. Figure 9 shows the application of (60) to zooming. On can observe that total variation based zooming leads to a superresolved 
image with sharp boundaries whereas standard bicubic interpolation does not preserve sharp boundaries.

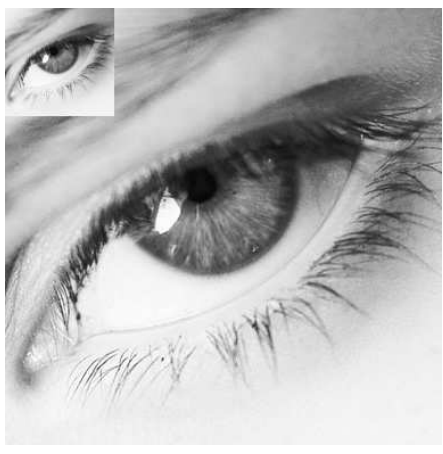

(a) Original images

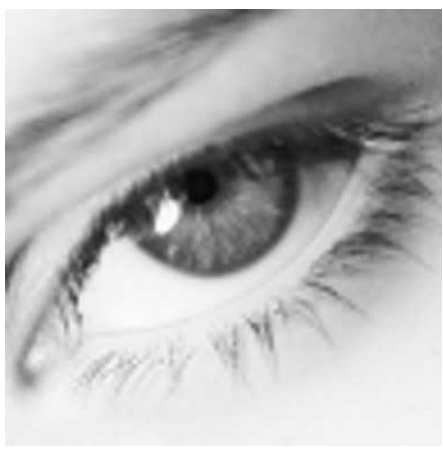

(b) Bicubic interpolation

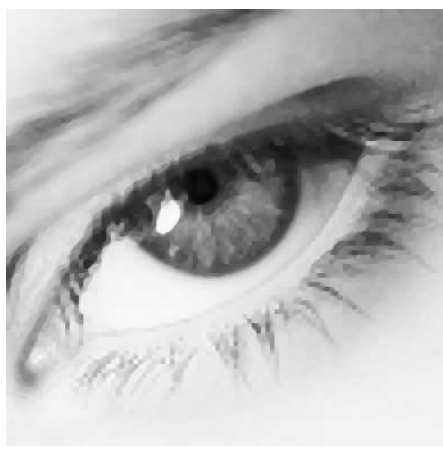

(c) TV-zooming

Figure 9: Image zooming using total variation regularization. (a) shows the original image and a by a factor of 4 downsampled version. (b) is the result of zooming by a factor of 4 using bicubic interpolation. (c) is the result of the total variation based zooming model. One can see that total variation based zooming yields much sharper image edges.

\subsection{Total variation with $L^{1}$ data fidelity term}

Similar to the $(R O F)$ model, the $T V-L^{1}$ model $[61,25,12]$ is defined as the variational problem

$$
\min _{u}\left\{\int_{\Omega}|D u|+\lambda \int_{\Omega}|u-f| d x\right\}
$$

The difference compared to the $(R O F)$ model is that the squared $L^{2}$ data fidelity term has been replaced by the $L^{1}$ norm. Although the change is small, the $T V-L^{1}$ model offers some desirable properties. First, it turns out that the $T V-L^{1}$ model is more effective than the $(R O F)$ model in removing impulse noise (e.g. salt and pepper noise) [61]. Second, the $T V-L^{1}$ model is contrast invariant. This means that, if $u$ is a solution of (62) for a certain input image $f$, then $c u$ is also a solution for $c f$ for $c \in \mathbb{R}^{+}$. Therefore the $T V-L^{1}$ model has a strong geometrical meaning which makes it useful for scale-driven feature selection and denoising of shapes.

Being not strictly convex, computing a minimizer of the $T V-L^{1}$ model is a hard task. Several methods have been proposed to compute an approximate minimizer using fixed point iterations based on smoothing [71] or quadratic splitting 
techniques [12]. Recently, an augmented Lagrangian method has been proposed to compute the exact minimizer of the $T V-L^{1}$ model [32]. In order to apply the proposed modified extragradient method to solve the $T V-L^{1}$ model, we again rewrite it in terms of a saddle-point problem (55)

$$
\min _{u} \max _{p, q}\langle p, D u\rangle+\langle q, u-f\rangle-I_{\left\{\|p\|_{\infty} \leq 1\right\}}-I_{\left\{|q|_{\infty} \leq \lambda\right\}},
$$

which can then be solved using the iterates (57).

Figure 4.2 shows the resoration of an image containing impulse noise (e.g. salt and pepper noise). As expected the $(R O F)$ model can not restore the image without loosing fine scale details. On the other hand, the $T V-L^{1}$ model does not give too much weight to the outliers and hence leads to much better results. This shows that it is important to use a data term which matches the expected noise model.

\subsection{Variational models with possibly nonconvex data terms}

The approach in this section is described with more details in the paper [64]. Let us consider the problem of finding the minimizer of an energy functional $F: L^{1}(\Omega) \rightarrow$ $[0, \infty]$ of the form

$$
\min _{u}\left\{F(u)=\int_{\Omega} f(x, u(x), \nabla u(x)) d x\right\}
$$

where $\Omega$ is a $d$-dimensional bounded open subset of $\mathbb{R}^{N}$ and $u: \Omega \rightarrow \mathbb{R}$ is an unknown scalar function. For $d=2, \Omega$ is usually assumed to be a rectangular image domain. The Lagrangian $f(x, t, p)$ is the "core" of the energy functional and is used to model the characteristics of the energy functional. We will assume here that $f(x, t, p)$ is continuous in $(x, t)$, and convex in $p$, but not necessarily in $t$.

\subsubsection{Convex representation}

We can introduce a general theoretical framework which is quite classical in the calculus of variations, although not so well-known. The basic concept is the idea of cartesian currents $[38,39]$, which consists in taking the whole graph $(x, u(x))$ of a function as the "object" to optimize upon, rather than the function $u$ itself. It is related to the so-called theory of calibration, which was recently brought back to light by Alberti et al. in [2], as an approach to characterize the minimizers of the Mumford-Shah functional [56] by an implicit (and new) convex representation: it 


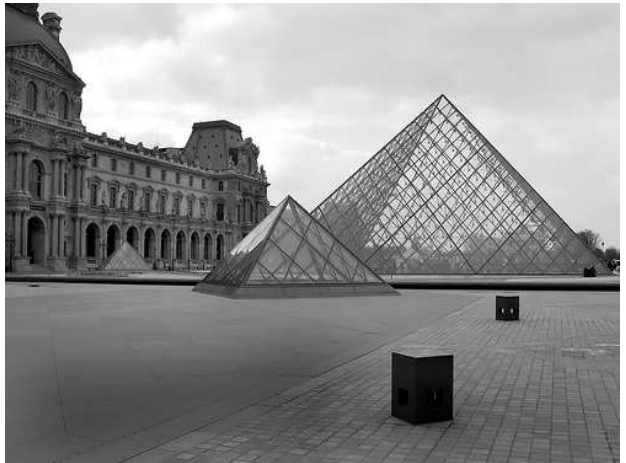

(a) Original image

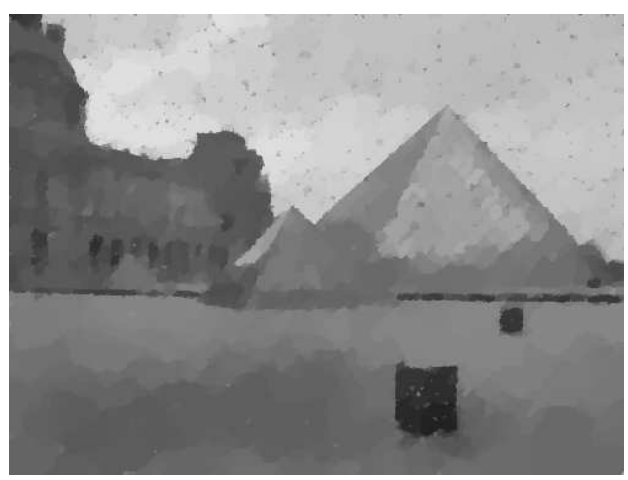

(c) $(R O F)$

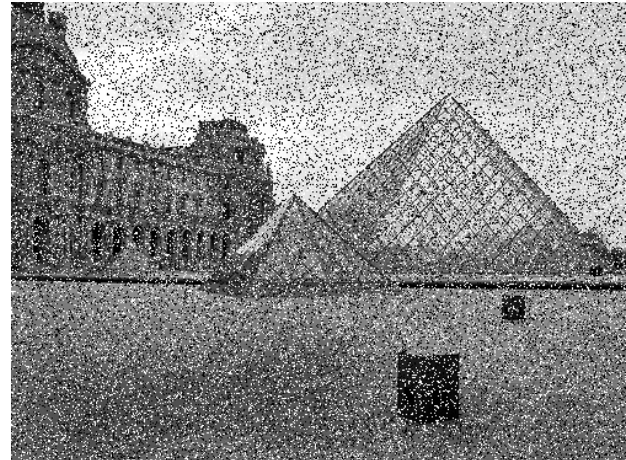

(b) Noisy image

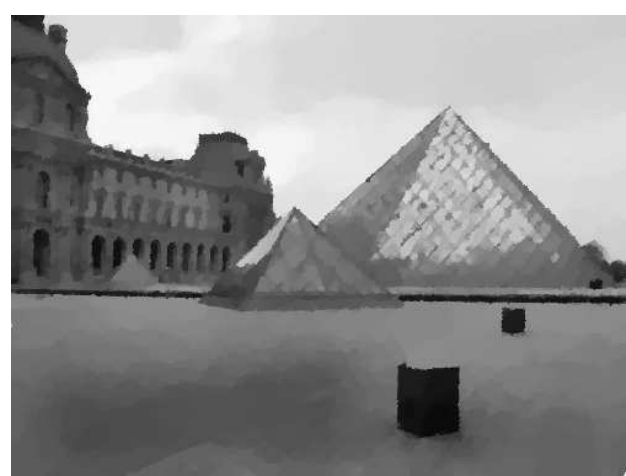

(d) $T V-L^{1}$

Figure 10: Image denoising in the case of impulse noise. (a) shows the clean image and (b) is a noisy version which has been corrupted by $25 \%$ salt and pepper noise. (c) is the result of the $(R O F)$ model. (d) is the result of the $T V-L^{1}$ model. Note that the $T V-L^{1}$ model is able to remove the noise while still preserving some small details.

allows to actually characterize (some) minimizers of the Mumford-Shah functional by means of divergence free vector in higher dimensions.

Let us start by considering the subgraph of the function $u(x)$, which is the collection of all points lying below the function value $u(x)$. Fig. 11 shows an example for a one-dimensional function $u(x)$ where the subgraph is represented as the gray area. We also introduce the function $\mathbf{1}_{u}(x, t): \Omega \times \mathbb{R} \rightarrow\{0,1\}$ which is the characteristic function of the subgraph of $u(x)$ :

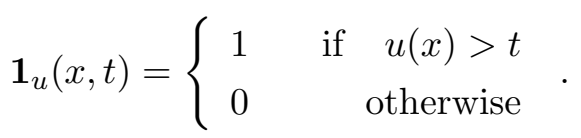




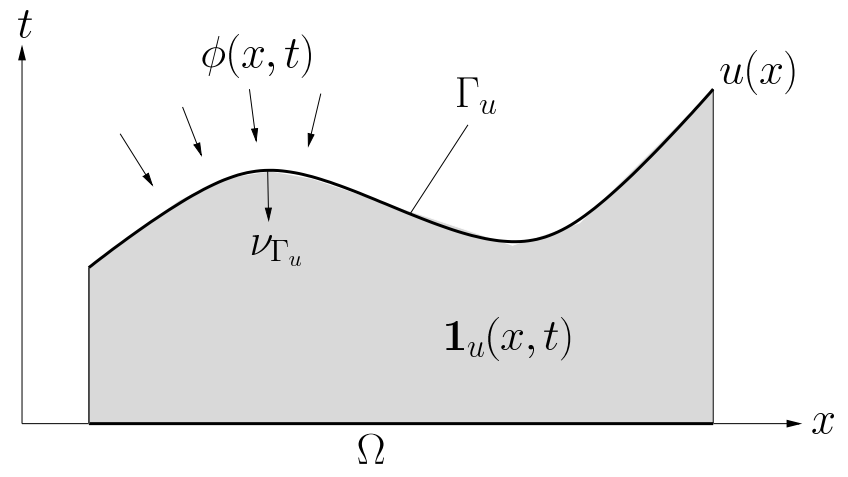

Figure 11: A one-dimensional function $u(x)$, its two-dimensional subgraph $\mathbf{1}_{u}$ and the vector field $\phi(x, t)$. The function $\mathbf{1}_{u}$ is supposed to be equal to 1 in the gray area and 0 outside.

Furthermore let us denote by $\Gamma_{u}$ the boundary of $\mathbf{1}_{u}(x, t)$. For the sake of simplicity, we assume first that $u$ is smooth.

The key idea is now to consider the flux of a vector field $\phi=\left(\phi^{x}, \phi^{t}\right): \Omega \times \mathbb{R} \rightarrow$ $\mathbb{R}^{N} \times \mathbb{R}$ through the boundary $\Gamma_{u}$.

$$
\Phi=\int_{\Gamma_{u}} \phi \cdot \nu_{\Gamma_{u}} d \mathcal{H}^{N},
$$

where $\mathcal{H}^{N}$ denotes the $N$-dimensional Hausdorff measure. $\nu_{\Gamma_{u}}$ denotes the inner unit normal to $\Gamma_{u}$ which is given by

$$
\nu_{\Gamma_{u}}=\frac{1}{\sqrt{1+|\nabla u(x)|^{2}}}\left(\begin{array}{c}
\nabla u(x) \\
-1
\end{array}\right)
$$

Alternatively, since we have $D \mathbf{1}_{u}=\nu_{\Gamma_{u}} \cdot \mathcal{H}^{N}$ on $\Gamma_{u}$, the flux can be written as

$$
\Phi=\int_{\Gamma_{u}} \phi \cdot \nu_{\Gamma_{u}} d \mathcal{H}^{N}=\int_{\Omega \times \mathbb{R}} \phi \cdot D \mathbf{1}_{u},
$$

where the expression $D \mathbf{1}_{u}$ denotes the distributional derivative of $\mathbf{1}_{u}$, which is, in an integral sense, also well defined for characteristic functions. In the following, it will turn out that by choosing an appropriate vector field $\phi, F(u)$ can be expressed as the maximal flux of $\phi$ through $\Gamma_{u}$.

Theorem 14. For any function $u \in W^{1,1}(\Omega ; \mathbb{R})$ the functional

$$
F(u)=\int_{\Omega} f(x, u(x), \nabla u(x)) d x,
$$


with $f(x, t, p)$ being continuous and positive in $t$ and convex in $p$, can be written as the higher dimensional convex functional

$$
\mathcal{F}\left(\mathbf{1}_{u}\right):=\sup _{\phi \in \mathcal{K}} \int_{\Omega \times \mathbb{R}} \phi \cdot D \mathbf{1}_{u},
$$

where the convex set $\mathcal{K}$ is given by

$$
\begin{aligned}
\mathcal{K}=\left\{\phi=\left(\phi^{x}, \phi^{t}\right) \in C_{0}\left(\Omega \times \mathbb{R} ; \mathbb{R}^{N} \times \mathbb{R}\right):\right. & \\
\phi^{t}(x, t) & \left.\geq f^{*}\left(x, t, \phi^{x}(x, t)\right), \forall x, t \in \Omega \times \mathbb{R}\right\} .
\end{aligned}
$$

Here, $f^{*}\left(x, t, p^{*}\right)$ denotes the Legendre-Fenchel conjugate (or convex conjugate) of $f(x, t, p)$ with respect to the last variable $p$, see Definition 3.3. Now, $f(x, t, p)$ being convex and lower semi continuous in $p$, Theorem 8 yields the equality $f^{* *}=f$.

Theorem 14 essentially states, that the potentially (but not necessarily) non convex functional (64) of a scalar function in dimension $N$ can be rewritten as a convex functional in dimension $N+1$. Moreover, it is seen from the definition that this convex functional is "a sort of" total variation, and essentially has the same structure. To sum up, we have recast problem (64) as the minimization of a modified (nonuniform, anisotropic) perimeter, and the new problem is similar to (16). It is remarkable that this works for functions $f(x, u(x), \nabla u(x))$ with a quite arbitrary behavior in $u(x)$ (although continuous, in that variable). On the other hand, this comes along with an increased computational complexity, since we added a dimension to the problem. ${ }^{4}$

Proof. Let us sketch the proof of Theorem 14. We first check that for any $\phi \in \mathcal{K}$, we have

$$
F(u) \geq \int_{\Omega \times \mathbb{R}} \phi \cdot D \mathbf{1}_{u} .
$$

Indeed, using (68) and the definition of the inner unit normal (67), the flux can be rewritten as

$$
\begin{array}{r}
\int_{\Omega \times \mathbb{R}} \phi \cdot D \mathbf{1}_{u}=\int_{\Gamma_{u}} \phi(x, t) \cdot\left(\begin{array}{c}
\nabla u(x) \\
-1
\end{array}\right) \frac{d \mathcal{H}^{N}(x, t)}{\sqrt{1+|\nabla u(x)|^{2}}} \\
=\int_{\Omega} \phi^{x}(x, u(x)) \cdot \nabla u(x)-\phi^{t}(x, u(x)) d x
\end{array}
$$

\footnotetext{
${ }^{4} \mathrm{And}$, in fact, it is not completely surprising if one thinks first of the case $N=0 \ldots$
} 
as $\sqrt{1+|\nabla u(x)|^{2}}$ is nothing else as the Jacobian of the change of variable $\Gamma_{u} \ni$ $(x, t) \mapsto x \in \Omega$. Since $\phi \in \mathcal{K}$, it follows

$$
\int_{\Omega \times \mathbb{R}} \phi \cdot D \mathbf{1}_{u} \leq \int_{\Omega} \phi^{x}(x, u(x)) \cdot \nabla u(x)-f^{*}\left(x, t, \phi^{x}(x, u(x))\right) d x,
$$

which is less than $F(u)$ by definition of the convex conjugate $f^{*}$. This shows (72).

The proof that the supremum is actually $F(u)$, that is, of $(70)$, is more technical. Essentially, one would need to choose $\phi^{x}(x, u(x))=\nabla_{p} f(x, u(x), \nabla u(x))$ at the point $(x, u(x))$ (since $p^{*}=\nabla_{p} f(x, t, p)$ reaches the maximum in $\max _{p}\langle q, p\rangle-$ $f(x, t, p)$, at least when $f$ is differentiable at $p$, see Prop. 3.5), and $\phi^{t}(x, u(x))=$ $f^{*}\left(x, t, \phi^{x}(x, u(x))\right)$. If $f, u$ are smooth enough (essentially, $C^{1}$ ), then such a choice can be performed. In other cases, it is shown that one can build a continuous field $\phi \in \mathcal{K}$ such that the flux (68) is arbitrarily close to $F(u)$.

Remark 4.1. In fact, the theorem still holds for $u \in B V(\Omega)$ a bounded variation function, and a Lagrangian $f(x, t, p)$ with linear growth (in $p$ ) at $\infty$, with a similar proof. It can also be extended to Lagrangians which take the value $+\infty$, such as illustrated in Fig. 12(c), with some additional regularity assumptions in $x, t$.

We have now transformed the problem of computing the minimizer of (64) into computing the minimizer of

$$
\min _{\mathbf{1}_{u}}\left\{\mathcal{F}\left(\mathbf{1}_{u}\right)=\sup _{\phi \in \mathcal{K}} \int_{\Omega \times \mathbb{R}} \phi \cdot D \mathbf{1}_{u}\right\} .
$$

Minimization in (74) is carried out over binary functions, which comprise a non convex set. Therefore we replace the function $\mathbf{1}_{u}$ in (74) by a more general function $v \in \mathcal{C}$, where the convex set $\mathcal{C}$ is given by

$$
\mathcal{C}=\left\{v \in B V(\Omega \times \mathbb{R} ;[0,1]): \lim _{t \rightarrow-\infty} v(x, t)=1, \lim _{t \rightarrow+\infty} v(x, t)=0\right\} .
$$

Hence we consider the relaxed problem

$$
\min _{v \in \mathcal{C}}\left\{\mathcal{F}(v):=\sup _{\phi \in \mathcal{K}} \int_{\Omega \times \mathbb{R}} \phi \cdot D v\right\} .
$$

Using this relaxation we essentially minimize the convex envelope $\mathcal{F}(v)$ of $\mathcal{F}\left(\mathbf{1}_{u}\right)$. 


\subsubsection{Convex relaxation}

Our intention is still to solve the binary problem. Hence, the question remains in which sense the minimizers of (76) and (74) are related? Indeed, one can show that a simple thresholding produces a solution of (74) from one of (76). This is summarized by the following result, which generalizes Proposition 2.1.

Proposition 4.2. Let $v^{*}$ by a global minimizer of (76). Then for any $s \in[0,1)$ the characteristic function $\mathbf{1}_{\left\{v^{*}>s\right\}}$ is also a global minimizer of (74).

Proof. The proof is the same as the proof of Prop. 2.1, as soon as one has observed that $\mathcal{F}$ satisfies the generalized co-area formula:

$$
\mathcal{F}(v)=\int_{-\infty}^{+\infty} \mathcal{F}\left(\mathbf{1}_{\{v>s\}}\right) d s .
$$

This follows from the fact that $\mathcal{F}$ can be represented as

$$
\mathcal{F}(v)=\int_{\Omega \times \mathbb{R}} h(x, t, D v)=\int_{\Omega \times \mathbb{R}} h\left(x, t, \frac{D v}{|D v|}\right)|D v|
$$

where $h$ is the convex, l.s.c. and one-homogeneous function of $D v=\left(D^{x} v, D^{t} v\right)$ defined as the support function of the convex set $\left\{\phi=\left(\phi^{x}, \phi^{t}\right): \phi^{t} \geq f^{*}\left(x, t, \phi^{x}\right)\right\}$, for any $(x, t)$ :

$$
h(x, t, D v):=\sup _{\phi^{t} \geq f^{*}\left(x, t, \phi^{x}\right)} \phi \cdot D v
$$

This function is shown to be nothing else as:

$$
h(x, t, D v)= \begin{cases}\left|D^{t} v\right| f\left(x, t, D^{x} v\right) /\left|D^{t} v\right| & \text { if } D^{t} v<0 \\ f^{\infty}\left(x, t, D^{x} v\right) & \text { if } D^{t} v=0 \\ +\infty & \text { if } D^{t} v>0\end{cases}
$$

where $f^{\infty}\left(x, t, p^{x}\right):=\lim _{\lambda \rightarrow+\infty} f\left(x, t, \lambda p^{x}\right) / \lambda$ is the recession function of $f$, see for instance $[28,39]$.

Hence, for any $v$, if we let $\nu_{v}=D v /|D v|$ (the Besicovitch derivative of the measure $D v$ with respect to its variation $|D v|)$, we have, using the standard co-area formula for $B V$ functions (in a form which is more general than $(C A)[36,34,74,7]$ ):

$$
\begin{aligned}
\mathcal{F}(v) & =\int_{\Omega \times \mathbb{R}} h\left(x, t, \nu_{v}(x, t)\right)|D v|=\int_{-\infty}^{+\infty} \int_{\Omega \times \mathbb{R}} h\left(x, t, \nu_{v}(x, t)\right)\left|D \mathbf{1}_{\{v>s\}}\right| d s \\
= & \int_{-\infty}^{+\infty} \int_{\Omega \times \mathbb{R}} h\left(x, t, D \mathbf{1}_{\{v>s\}} /\left|D \mathbf{1}_{\{v>s\}}\right|\right)\left|D \mathbf{1}_{\{v>s\}}\right| d s=\int_{-\infty}^{+\infty} \mathcal{F}\left(\mathbf{1}_{\{v>s\}}\right) d s
\end{aligned}
$$


where we have used the fact that $\mathcal{H}^{N-1}$-a.e. on the boundary of $\{v>s\}, \nu_{v}=$ $\nu_{\{v>s\}}=D 1_{\{v>s\}} /\left|D 1_{\{v>s\}}\right|$, that is, the gradient of $v$ is normal to its level lines.

\subsubsection{Numerical resolution}

After a suitable discretization (as described in Section 3.1), problem (76), which is of the form (55), can be solved for instance by algorithm (57).

The most complicated step requires to project onto the (discretized version of the) set $\mathcal{K}$ defined in (71). Let us describe now a few examples. We will assume that $f(x, t, p)$ has the form $g(x, t)+h(p)$. Then, $\mathcal{K}$ is reduced to

$$
\left\{\phi: \phi^{t}(x, t) \geq h^{*}\left(\phi^{x}(x, t)\right)-g(x, t)\right\}
$$

and we essentially need to know how to project onto the convex set $\left\{q=\left(q^{x}, q^{t}\right)\right.$ : $\left.q^{t} \geq h^{*}\left(q^{x}\right)\right\}$.

Quadratic regularization If $h(p)=|p|^{2} / 2$, then $h^{*}(q)=|q|^{2} / 2$ as well, and we need to know how to project some $q_{0}=\left(q_{0}^{x}, q_{0}^{t}\right)$ onto $K=\left\{q=\left(q^{x}, q^{t}\right): q^{t} \geq\right.$ $\left.\left|q^{x}\right|^{2} / 2\right\}$, see Fig. 12(a).

If $q_{0}$ does not satisfy the constraint, that is, $q_{0}^{t}<\left|q_{0}^{x}\right|^{2} / 2$, we need to project $q_{0}$ onto the paraboloid $q^{t}=\left|q^{x}\right|^{2} / 2$. Hence we must solve the following unconstrained optimization problem

$$
\min _{q}\left\{\frac{\left|q-q_{0}\right|^{2}}{2}-\lambda\left(q^{t}-\frac{\left|q^{x}\right|^{2}}{2}\right)\right\}
$$

where $\lambda$ is a Lagrange multiplier for the equality constraint $q^{t}-\left|q^{x}\right|^{2} / 2=0$. The optimaliy conditions of (81) are given by

$$
\begin{aligned}
q^{x}-q_{0}^{x}+\lambda q^{x} & =0 \\
q^{t}-q_{0}^{t}-\lambda & =0 \\
q^{t}-\frac{\left|q^{x}\right|^{2}}{2} & =0,
\end{aligned}
$$

After eliminating $q^{t}$ and $q^{x}$, we arrive at the following cubic equation for $\lambda$ :

$$
\lambda^{3}+\lambda^{2}\left(q_{0}^{t}+2\right)+\lambda\left(2 q_{0}^{t}+1\right)+q_{0}^{t}-\frac{\left|q_{0}^{x}\right|^{2}}{2}=0 .
$$




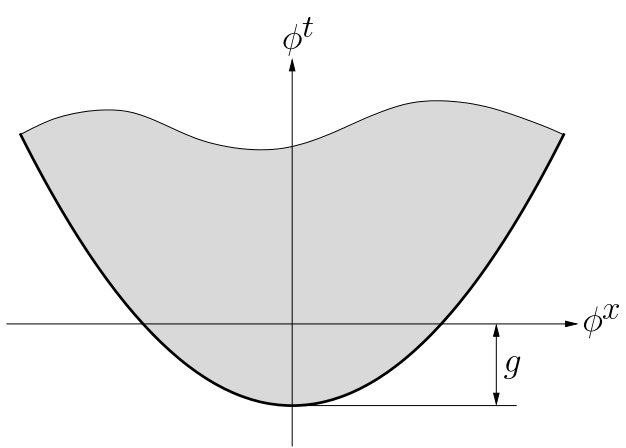

(a) $h(p)=|p|^{2} / 2$

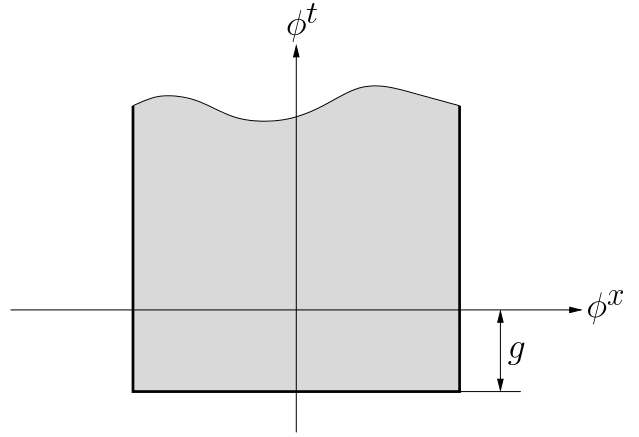

(b) $h(p)=|p|$

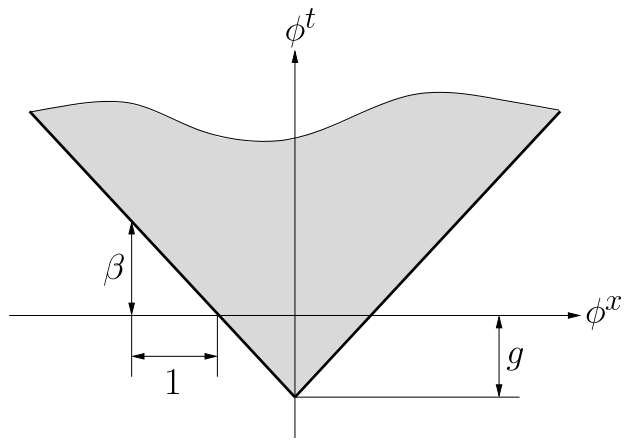

(c) $h(p)=0$ if $|p| \leq \beta$ and $+\infty$ else

Figure 12: The sets $\left\{q^{t} \geq h^{*}\left(q^{x}\right)\right\}$ for various $h$ : (a) Quadratic, (b) Total Variation, (c) Lipschitz regularization.

Instead of using a direct cubic solver for (83) we utilize Newton's method. We choose a starting point $\left.\lambda^{0}=\max \left\{0,-\left(2 q_{0}^{t}+1\right) / 3\right)\right\}+1$ and let for each $n \geq 0$

$$
\lambda^{n+1}=\lambda^{n}-\frac{\lambda^{3}+\lambda^{2}\left(q_{0}^{t}+2\right)+\lambda\left(2 q_{0}^{t}+1\right)+q_{0}^{t}-\frac{\left|q_{0}^{x}\right|^{2}}{2}}{3 \lambda^{2}+2 \lambda\left(q_{0}^{t}+2\right)+2 q_{0}^{t}+1} .
$$

We found this scheme to have a quite fast convergence. We never experienced more than $10-20$ iterations to achieve a reasonable accuracy. Then, after computing the solution of (83), the solution of the projection is given by

$$
q=\left(\frac{q_{0}^{x}}{1+\lambda}, q_{0}^{t}+\lambda\right)
$$

Total variation regularization In case $h(p)=|p|$, then $h^{*}(q)=0$ for $|q| \leq 1$, and $+\infty$ else, and the projection of $q_{0}$ onto the convex $\left\{q=\left(q^{x}, q^{t}\right): q^{t} \geq 0,\left|q^{x}\right| \leq\right.$ 


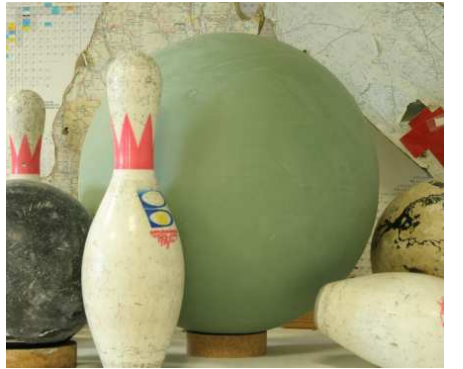

(a) Left input image

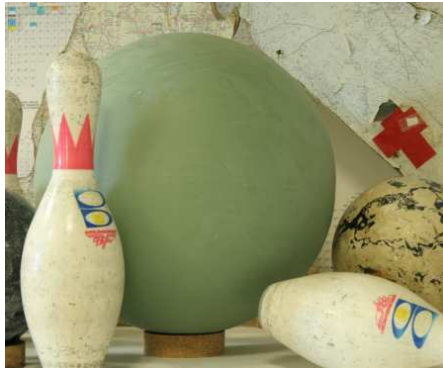

(b) Right input image

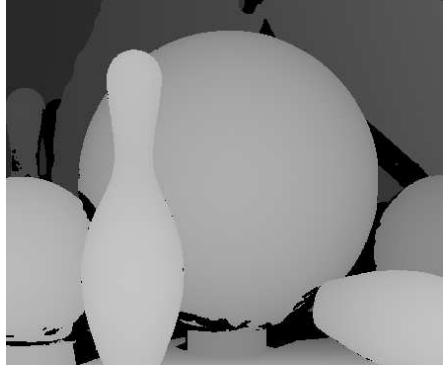

(c) "True" disparity

Figure 13: Rectified stereo image pair and the ground truth disparity, where black pixels correspod to unknown disparity values.

1\}, see Fig. 12(b), is simply given by:

$$
q=\left(\frac{q_{0}^{x}}{\max \left\{1,\left|q_{0}^{x}\right|\right\}}, \max \left\{0, q_{0}^{t}\right\}\right) .
$$

Lipschitz constraint One advantage of this approach is that a Lipschitz constraint is quite easy to enforce. We consider $h(p)=0$ if $|p| \leq L,+\infty$ else. Then, the convex conjugate of $h$ is simply $h^{*}(q)=L|q|$, and we just need to know how to project $q_{0}$ onto the convex cone $\left\{q^{t} \geq L\left|q^{x}\right|\right\}$, see Fig. 12(c). This projection is of course straightforward: is given by

$$
q=\left(\mu \frac{q_{0}^{x}}{\left|q_{0}^{x}\right|}, \mu L\right)
$$

where $\mu$ is given by

$$
\mu=\frac{\max \left\{0,\left|q_{0}^{x}\right|+L q_{0}^{t}\right\}}{1+L^{2}}
$$

Example Figure 14 shows three examples of stereo reconstruction using the three different regularizers described above. Of course, only the total variation performs well in this context. As expected, the Lipschitz constraint limits the slope of the solution, while the quadratic contstraint also oversmooths. The best compromise would be to take $h$ a Huber function, quadratic near zero and linear for large values, see [64] for this example. 


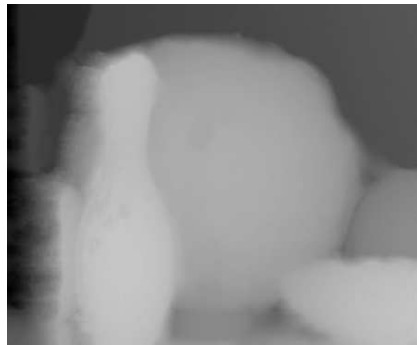

(a)

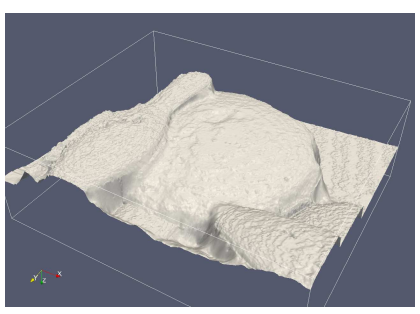

(d)

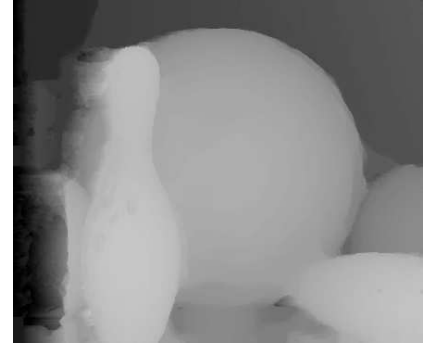

(b)

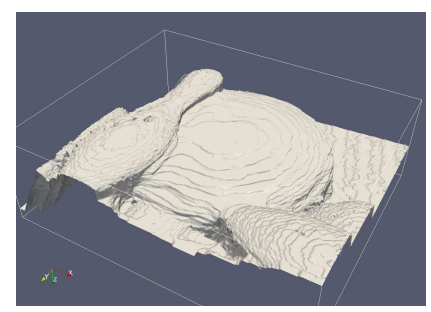

(e)

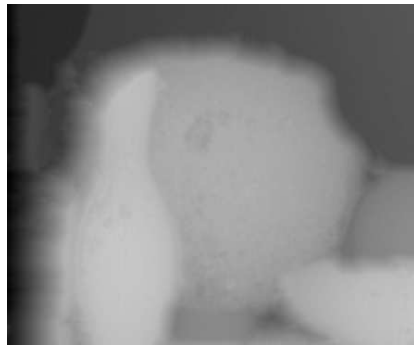

(c)

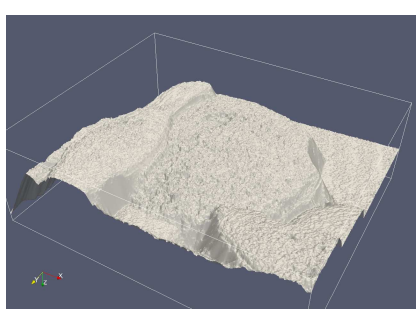

(f)

Figure 14: Application of different convex regularity terms to disparity estimation. First column: Quadratic regularization, second column: Total Variation regularization, third column: Lipschitz regularization.

\subsection{The minimal partition problem}

Consider now the problem of finding a segmentation into $k$ sets of a domain $\Omega \subset \mathbb{R}^{N}$, which minimizes the total interface between the sets, as in the piecewise constant Mumford-Shah problem:

$$
\min _{\left(R_{i}\right)_{i=1}^{k},\left(c_{i}\right)_{i=1}^{k}} \frac{\lambda}{2} \sum_{i=1}^{k} \operatorname{Per}\left(R_{i} ; \Omega\right)+\frac{1}{2} \sum_{i=1}^{k} \int_{R_{i}}\left|g(x)-c_{i}\right|^{2} d x
$$

where $c_{i} \in \mathbb{R}$ and where the regions $\left(R_{i}\right)_{i=1}^{k}$ form a partition of $\Omega$, that is, $R_{i} \cap R_{j}=\emptyset$ if $i \neq j$ and $\bigcup_{i=1}^{k} R_{i}=\Omega$. It corresponds to the best least square approximation of $g$ with a piecewise constant function $u=\sum_{i} c_{i} \chi_{R_{i}}$, whith the total perimeter of the partition as the cost of a particular approximation (the total number of set $k$ should in theory be free, however it is always possible to bound it from above if $g$ is bounded, so that there is no loss of generality in keeping it fixed). Of course, given the partition $\left(R_{i}\right)_{i=1}^{k}$, the optimal constant $c_{i}=\left(1 /\left|R_{i}\right|\right) \int_{R_{i}} g d s$ is the average value of $g$ on $R_{i}$ for each $i=1, \ldots, k$. On the other hand, finding the minimum of (89) with respect to the partition $\left(R_{i}\right)_{i=1}^{k}$ is a hard task, even for fixed values $\left(c_{i}\right)_{i=1}^{k}$. It 
is known that its discrete counterpart (the Pott's model) is NP-hard, so that it is unlikely that (89) has a simple convex representation, at least without increasing drastically the number of variables.

We will show that one can consider simple convex approximations the interface term in (89) which can be actually minimized, and in many cases provide a solution of the original problem (although nothing of this kind is know in general).

Let us introduce $v_{i}=\chi_{R_{i}} \in B V(\Omega)$ : the functions $v_{i}$ satisfy $v_{i}(x) \in\{0,1\}$ and $\sum_{i=1}^{k} v_{i}(x)=1$ a.e. in $\Omega$. Moreover, letting $\mathbf{v}=\left(v_{1}, \ldots, v_{k}\right) \in B V\left(\Omega ; \mathbb{R}^{k}\right)$, we see that

$$
J_{\mathbf{v}}=\bigcup_{i=1}^{l} J_{v_{i}}=\bigcup_{i=1}^{l} \Omega \cap \partial^{*} R_{i}
$$

and the total surface of the interface is

$$
\mathcal{H}^{N-1}\left(J_{\mathbf{v}}\right)=\frac{1}{2} \sum_{i=1}^{k} \operatorname{Per}\left(R_{i} ; \Omega\right)
$$

since in the right-hand side, the common boundary of $R_{i}$ and $R_{j}$ is counted twice for all $i \neq j$.

We can therefore define the partition functional as

$$
\mathcal{J}(\mathbf{v})= \begin{cases}\mathcal{H}^{N-1}\left(J_{\mathbf{v}}\right) & \text { if } \mathbf{v} \in B V\left(\Omega ;\{0,1\}^{k}\right) \text { with } \sum_{i=1}^{k} v_{i}=1 \text { a.e. } \\ +\infty & \text { else. }\end{cases}
$$

Then, the best convex approximation of $\mathcal{J}$ should be its convex l.s.c. envelope $\mathcal{J}^{* *}$ (in $L^{2}\left(\Omega ; \mathbb{R}^{k}\right)$ ), as defined in Definition 3.3:

$$
\mathcal{J}^{* *}(\mathbf{v})=\sup _{\mathbf{w} \in L^{2}\left(\Omega ; \mathbb{R}^{k}\right)}\langle\mathbf{w}, \mathbf{v}\rangle-\mathcal{J}^{*}(\mathbf{w})
$$

where

$$
\mathcal{J}^{*}(\mathbf{w})=\sup _{\mathbf{v} \in L^{2}\left(\Omega ; \mathbb{R}^{k}\right)}\langle\mathbf{w}, \mathbf{v}\rangle-\mathcal{J}(\mathbf{v}) .
$$

However, this provides an abstract definition of $\mathcal{J}^{* *}$ but does not say how to actually compute it or minimize it. It is possible, though, to show that the domain of $\mathcal{J}^{* *}$ is

$$
\operatorname{dom} \mathcal{J}^{* *}=\left\{\mathbf{v} \in B V\left(\Omega ;[0,1]^{k}\right), \sum_{i=1}^{k} v_{i}=1 \text { a.e. in } \Omega\right\},
$$

see [22] where a different representation is used but this can be deduced by a simple change of variable. 
To be able to numerically solve the problem, one should find a convex, l.s.c. functional $J \leq \mathcal{J}^{5}$ with a particular form, which can be handled and provide a problem that can be actually solved. A typical form is $J(\mathbf{v})=\int_{\Omega} F(x, D \mathbf{v})$ for $F(x, p)$ some function, convex in $p$ and measurable in $x$. Moreover, one should at least require that $J=\mathcal{J}$ on its domain (that is, on binary functions $\mathbf{v} \in\{0,1\}^{k}$ with $\sum_{i} v_{i}=1$ ). Eventually, one should try to find the largest possible $J$ in this class, so that it becomes more likely that a solution of

$$
\min _{\mathbf{v}} \lambda J(v)+\frac{1}{2} \int_{\Omega} \sum_{i=1}^{k} v_{i}(x)\left|g(x)-c_{i}\right|^{2} d x
$$

is itself binary (in the domain of $\mathcal{J}$ ), and therefore provides a minimizer of (89) for fixed $\left(c_{i}\right)_{i=1}^{k}$.

Several choices have been proposed in the literature. In [72], it is proposed to use simple LP-relaxation exactly as for the multiway cut problem in the discrete literature [1]. Hence one just lets

$$
J(v)=\frac{1}{2} \sum_{i=1}^{k} \int_{\Omega}\left|D v_{i}\right| \quad \text { if } \mathbf{v} \in B V\left(\Omega ;[0,1]^{k}\right),
$$

and $+\infty$ else, which naturally extends (90) to the domain of $\mathcal{J}^{* *}$. However, it can be shown that this relaxation is too small, see [22, Prop. A.1.] and Figure 15(b).

On the other hand, [47] propose to use the vectorial total variation (appropriately rescaled), which is defined exactly like in $(T V)$, that is,

$$
\begin{aligned}
J(v)=\frac{1}{\sqrt{2}} \int_{\Omega}|D \mathbf{v}| & \\
& =\frac{1}{\sqrt{2}} \sup \left\{-\int \mathbf{v} \cdot \operatorname{div} \phi: \phi \in C_{c}^{\infty}\left(\Omega ; \mathbb{R}^{N \times k}\right), \sum_{i, j} \phi_{i, j}^{2} \leq 1\right\} .
\end{aligned}
$$

Examples show that this does not perform much better than the previous choice for recovering triple junctions, see Figure 15(c).

The "best" possible choice, if of the form $\int_{\Omega} F(x, D \mathbf{v})$ for a convex, even function $F(x, p)$ (w.r. $p$ ), can be shown to be

$$
J(\mathbf{v})=\int_{\Omega} \Psi(D \mathbf{v})
$$

\footnotetext{
${ }^{5}$ hence $J \leq \mathcal{J}^{* *}$, since it is the largest convex, l.s.c. functional below $\mathcal{J}$.
} 


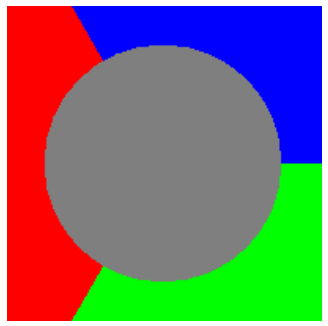

(a) Input image

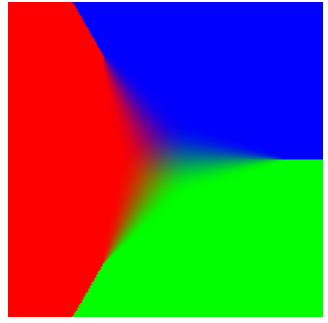

(b) Relaxation (92)

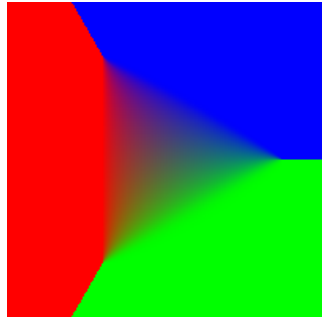

(c) Relaxation (93)

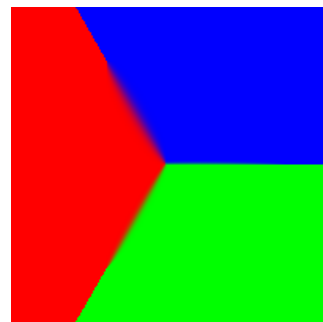

(d) Relaxation (94)

Figure 15: Triple junction experiment with $k=3$. (a) shows the input image with given boundary datum. (b) shows the result using the relaxation of [72], and (c) the relaxation of [47]. (d) shows the result of the proposed relaxation.

for $\Psi: \mathbb{R}^{N \times k} \rightarrow[0,+\infty]$ the convex, 1-homogeneous function given by

$$
\Psi(p)=\sup \left\{\sum_{i=1}^{k}\left\langle p_{i}, q_{i},\right\rangle:\left|p_{i}-p_{j}\right| \leq 1 \forall 1 \leq i<j \leq k\right\} .
$$

(Note in particular that $\Psi(p)=+\infty$ if $\sum_{i=1}^{k} p_{i} \neq 0$, which is not an issue since if $\mathbf{v} \in \operatorname{dom} \mathcal{J}^{* *}, \sum v_{i}=1$ hence $\sum_{i} D v_{i}=0$.) In our notation, for $p$ a vector in $\mathbb{R}^{N \times k}, p_{j}=\left(p_{i, j}\right)_{i=1}^{N}$ is a $N$-dimensional vector for each $j=1, \ldots, k$. Letting

$$
\mathcal{K}=\left\{-\operatorname{div} \phi: \phi \in C_{c}^{\infty}\left(\Omega ; \mathbb{R}^{N \times k}\right),\left|\phi_{i}(x)-\phi_{j}(x)\right| \leq 1 \forall x \in \Omega, 1 \leq i<j \leq k\right\},
$$

we can also define $J$ as the support function of $\mathcal{K}$ :

$$
J(\mathbf{v})=\sup _{\mathbf{w} \in \mathcal{K}}\langle\mathbf{w}, \mathbf{v}\rangle,
$$

which is a variant of $(T V)$ : it is again a sort of total variation and we can hope to minimize it with the techniques described in Section 3. This construction is related to the theory of "paired calibration" introduced in the 1990's by Lawlor-Morgan and Brakke [46, 18].

In order to minimize (91) using the techniques described in these notes, one first chooses to minimize alternatively with respect to the partition $\left(R_{i}\right)_{i=1}^{k}$, described by the function $\mathbf{v}$, and with respect to the constants $\left(c_{i}\right)_{i=1}^{k}$ (which, in general, should lead to a local minimizer, or even a mere critical point of the problem). Minimizing with respect to the $c_{i}$ 's is straightforward (the solution is simply the average of the data in the region $R_{i}$ ). The minimization with respect to $\mathbf{v}$ is performed, for instance, using Algorithm (57). A crucial step is the projection 


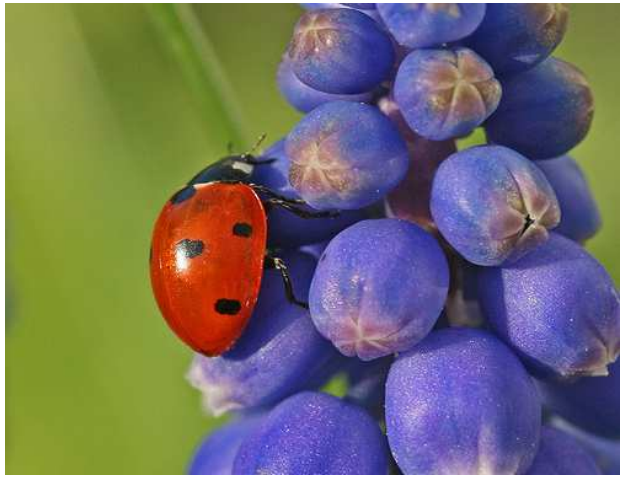

(a) Input image

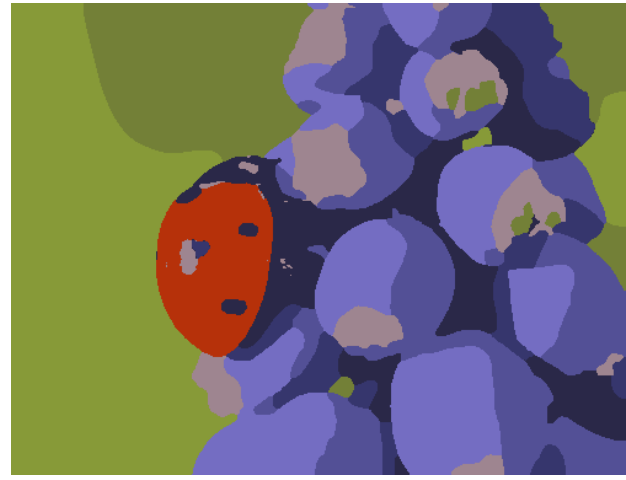

(b) Segmentation, $k=8$

Figure 16: Piecewise constant Mumford-Shah segmentation of a natural image. (a) shows the input image and (b) is the minimizer of energy (89). The mean color values $c_{i}$ of the partitions have been initialized using k-means clustering.

onto $K=\left\{p \in \mathbb{R}^{N \times k}:\left|p_{i}-p_{j}\right| \leq 1 \forall i, j\right\}$, whose $\Psi$ is the support function. This is performed by alternate projections, following Dykstra's algorithm for projecting onto intersection of convex sets [17].

Figure 15 shows the minimization of $J$ with a boundary datum which enforces the completion of three regions: we find a triple point with $120^{\circ}$ angles, as the theory expects. Observe that lower relaxations provide wrong results (with a relaxed energy strictly below the interfacial energy of the original problem). The next Figure 16 shows an example of a minimization of (91) done following this approach. (The numerical computations have been performed on a GPU.)

\section{A A proof of convergence}

We prove in this section Theorem 13, that is, a convergence estimate for the modified Douglas-Rachford (or extragradient) algorithm (57). The assumption that $x^{0}=\bar{x}^{0}$ can also be written $x^{-1}=x^{0}$ and $\bar{x}^{0}=2 x^{0}-x^{-1}$, which is consistent with the definition of $\bar{x}^{n+1}$ for $n \geq 0$. The proof which follows is heavily inspired by [57] (for the estimate) and [65] (for the convergence proof).

Proof. We have

$$
\begin{aligned}
& \partial F^{*}\left(y^{n+1}\right) \ni \frac{y^{n}-y^{n+1}}{\sigma}+A \bar{x}^{n} \\
& \partial G\left(x^{n+1}\right) \ni \frac{x^{n}-x^{n+1}}{\tau}-A^{*} y^{n+1}
\end{aligned}
$$


so that for any $(x, y) \in X \times Y$,

$$
\begin{aligned}
& F^{*}(y) \geq F^{*}\left(y^{n+1}\right)+\left\langle\frac{y^{n}-y^{n+1}}{\sigma}, y-y^{n+1}\right\rangle+\left\langle A \bar{x}^{n}, y-y^{n+1}\right\rangle \\
& G(x) \geq G\left(x^{n+1}\right)+\left\langle\frac{x^{n}-x^{n+1}}{\tau}, x-x^{n+1}\right\rangle-\left\langle y^{n+1}, A\left(x-x^{n+1}\right)\right\rangle .
\end{aligned}
$$

Summing both inequalities, it follows:

$$
\begin{gathered}
F^{*}(y)+G(x)+\frac{\left\|y-y^{n}\right\|^{2}}{2 \sigma}+\frac{\left\|x-x^{n}\right\|^{2}}{2 \tau} \geq \\
F^{*}\left(y^{n+1}\right)+G\left(x^{n+1}\right)+\left\langle A x^{n+1}-\bar{x}^{n}, y^{n+1}-y\right\rangle+\left\langle A x^{n+1}, y\right\rangle-\left\langle y^{n+1}, A x\right\rangle \\
+\frac{\left\|y-y^{n+1}\right\|^{2}}{2 \sigma}+\frac{\left\|x-x^{n+1}\right\|^{2}}{2 \tau}+\frac{\left\|y^{n}-y^{n+1}\right\|^{2}}{2 \sigma}+\frac{\left\|x^{n}-x^{n+1}\right\|^{2}}{2 \tau}
\end{gathered}
$$

Now:

$$
\begin{gathered}
\left\langle A\left(x^{n+1}-\bar{x}^{n}\right), y^{n+1}-y\right\rangle=\left\langle A\left(\left(x^{n+1}-x^{n}\right)-\left(x^{n}-x^{n-1}\right)\right), y^{n+1}-y\right\rangle \\
=\left\langle A\left(x^{n+1}-x^{n}, y^{n+1}-y\right\rangle-\left\langle A\left(x^{n}-x^{n-1}\right), y^{n}-y\right\rangle\right. \\
-\left\langle A\left(x^{n}-x^{n-1}\right), y^{n+1}-y^{n}\right\rangle \\
\geq\left\langle A\left(x^{n+1}-x^{n}\right), y^{n+1}-y\right\rangle-\left\langle A\left(x^{n}-x^{n-1}\right), y^{n}-y\right\rangle \\
-L\left\|x^{n}-x^{n-1}\right\|\left\|y^{n+1}-y^{n}\right\| .
\end{gathered}
$$

For any $\delta>0$, we have that (using $2 a b \leq \delta a^{2}+b^{2} / \delta$ for any $a, b$ )

$$
L\left\|x^{n}-x^{n-1}\right\|\left\|y^{n+1}-y^{n}\right\| \leq \frac{L \delta \tau}{2 \tau}\left\|x^{n}-x^{n-1}\right\|^{2}+\frac{L \sigma}{2 \delta \sigma}\left\|y^{n+1}-y^{n}\right\|^{2}
$$

and we choose $\delta=\sqrt{\sigma / \tau}$, so that $L \delta \tau=L \sigma / \delta=\sqrt{\sigma \tau} L<1$.

Summing the last inequality together with (95) and (96), we get that for any $x \in X$ and $y \in Y$,

$$
\begin{aligned}
& \frac{\left\|y-y^{n}\right\|^{2}}{2 \sigma}+\frac{\left\|x-x^{n}\right\|^{2}}{2 \tau} \geq \\
& {\left[\left\langle A x^{n+1}, y\right\rangle-F^{*}(y)+G\left(x^{n+1}\right)\right]-\left[\left\langle A x, y^{n+1}\right\rangle-F^{*}\left(y^{n+1}\right)+G(x)\right]} \\
& +\frac{\left\|y-y^{n+1}\right\|^{2}}{2 \sigma}+\frac{\left\|x-x^{n+1}\right\|^{2}}{2 \tau}+(1-\sqrt{\sigma \tau} L) \frac{\left\|y^{n}-y^{n+1}\right\|^{2}}{2 \sigma} \\
& +\frac{\left\|x^{n}-x^{n+1}\right\|^{2}}{2 \tau}-\sqrt{\sigma \tau} L \frac{\left\|x^{n-1}-x^{n}\right\|^{2}}{2 \tau} \\
& +\left\langle A\left(x^{n+1}-x^{n}\right), y^{n+1}-y\right\rangle-\left\langle A\left(x^{n}-x^{n-1}\right), y^{n}-y\right\rangle
\end{aligned}
$$


Let us now sum (97) from $n=0$ to $N-1$ : it follows that for any $x$ and $y$,

$$
\begin{aligned}
& \sum_{n=1}^{N}\left[\left\langle A x^{n}, y\right\rangle-F^{*}(y)+G\left(x^{n}\right)\right]-\left[\left\langle A x, y^{n}\right\rangle-F^{*}\left(y^{n}\right)+G(x)\right] \\
& +\frac{\left\|y-y^{N}\right\|^{2}}{2 \sigma}+\frac{\left\|x-x^{N}\right\|^{2}}{2 \tau}+(1-\sqrt{\sigma \tau} L) \sum_{n=1}^{N} \frac{\left\|y^{n}-y^{n-1}\right\|^{2}}{2 \sigma} \\
& +(1-\sqrt{\sigma \tau} L) \sum_{n=1}^{N-1} \frac{\left\|x^{n}-x^{n-1}\right\|^{2}}{2 \tau}+\frac{\left\|x^{N}-x^{N-1}\right\|^{2}}{2 \tau} \\
& \leq \frac{\left\|y-y^{0}\right\|^{2}}{2 \sigma}+\frac{\left\|x-x^{0}\right\|^{2}}{2 \tau}+\left\langle A\left(x^{N}-x^{N-1}\right), y^{N}-y\right\rangle
\end{aligned}
$$

where we have used $x^{-1}=x^{0}$. Now, as before,

$$
\left\langle A\left(x^{N}-x^{N-1}\right), y^{N}-y\right\rangle \leq\left\|x^{N}-x^{N-1}\right\|^{2} /(2 \tau)+\left(\tau \sigma L^{2}\right)\left\|y-y^{N}\right\|^{2} /(2 \sigma),
$$

and it follows

$$
\begin{aligned}
& \sum_{n=1}^{N}\left[\left\langle A x^{n}, y\right\rangle-F^{*}(y)+G\left(x^{n}\right)\right]-\left[\left\langle A x, y^{n}\right\rangle-F^{*}\left(y^{n}\right)+G(x)\right] \\
& +\left(1-\sigma \tau L^{2}\right) \frac{\left\|y-y^{N}\right\|^{2}}{2 \sigma}+\frac{\left\|x-x^{N}\right\|^{2}}{2 \tau}+(1-\sqrt{\sigma \tau} L) \sum_{n=1}^{N} \frac{\left\|y^{n}-y^{n-1}\right\|^{2}}{2 \sigma} \\
& +(1-\sqrt{\sigma \tau} L) \sum_{n=1}^{N-1} \frac{\left\|x^{n}-x^{n-1}\right\|^{2}}{2 \tau} \leq \frac{\left\|y-y^{0}\right\|^{2}}{2 \sigma}+\frac{\left\|x-x^{0}\right\|^{2}}{2 \tau}
\end{aligned}
$$

First we choose $(x, y)=(\hat{x}, \hat{y})$ a saddle-point in (98). Then, it follows from (56) that the first summation in (98) is non-negative, and point (a) in Theorem 13 follows. We then deduce from (98) and the convexity of $G$ and $F^{*}$ that, letting $x_{N}=\left(\sum_{n=1}^{N} x^{n}\right) / N$ and $y_{N}=\left(\sum_{n=1}^{N} y^{n}\right) / N$,

$$
\begin{aligned}
{\left[\left\langle A x_{N}, y\right\rangle-F^{*}(y)+G\left(x_{N}\right)\right]-\left[\left\langle A x, y_{N}\right\rangle\right.} & \left.-F^{*}\left(y_{N}\right)+G(x)\right] \\
& \leq \frac{1}{N}\left(\frac{\left\|y-y^{0}\right\|^{2}}{2 \sigma}+\frac{\left\|x-x^{0}\right\|^{2}}{2 \tau}\right)
\end{aligned}
$$

for any $(x, y) \in X \times Y$, which yields (59). Consider now a weak cluster point $\left(x^{*}, y^{*}\right)$ of $\left(x_{N}, y_{N}\right)$ (which is a bounded sequence, hence weakly compact). Since $G$ and $F^{*}$ are convex and l.s.c. they also are weakly l.s.c., and it follows from (99) that

$$
\left[\left\langle A x^{*}, y\right\rangle-F^{*}(y)+G\left(x^{*}\right)\right]-\left[\left\langle A x, y^{*}\right\rangle-F^{*}\left(y^{*}\right)+G(x)\right] \leq 0
$$


for any $(x, y) \in X \times Y$ : this shows that $\left(x^{*}, y^{*}\right)$ satisfies (56) and therefore is a saddle-point. We have shown point (b) in Theorem 13.

It remains to prove the convergence to a saddle point if the spaces $X$ and $Y$ are finite-dimensional. Point (a) establishes that $\left(x^{n}, y^{n}\right)$ is a bounded sequence, so that some subsequence $\left(x^{n_{k}}, y^{n_{k}}\right)$ converges to some limit $\left(x^{*}, y^{*}\right)$, strongly since we are in finite dimension. Observe that (98) implies that $\lim _{n}\left(x^{n}-x^{n-1}\right)=$ $\lim _{n}\left(y^{n}-y^{n-1}\right)=0$, in particular also $x^{n_{k}-1}$ and $y^{n_{k}-1}$ converge respectively to $x^{*}$ and $y^{*}$. It follows that the limit $\left(x^{*}, y^{*}\right)$ is a fixed point of the algorithm (57), hence a saddle point of our problem.

We can then take $(x, y)=\left(x^{*}, y^{*}\right)$ in $(97)$, which we sum from $n=n^{k}$ to $N-1$, $N>n_{k}$. We obtain

$$
\begin{gathered}
\frac{\left\|y^{*}-y^{N}\right\|^{2}}{2 \sigma}+\frac{\left\|x^{*}-x^{N}\right\|^{2}}{2 \tau}+(1-\sqrt{\sigma \tau} L) \sum_{n=n_{k}+1}^{N} \frac{\left\|y^{n}-y^{n-1}\right\|^{2}}{2 \sigma} \\
-\frac{\left\|x^{n_{k}}-x^{n_{k}-1}\right\|^{2}}{2 \tau}+(1-\sqrt{\sigma \tau} L) \sum_{n=n_{k}}^{N-1} \frac{\left\|x^{n}-x^{n-1}\right\|^{2}}{2 \tau}+\frac{\left\|x^{N}-x^{N-1}\right\|^{2}}{2 \tau} \\
+\left\langle A\left(x^{N}-x^{N-1}\right), y^{N}-y^{*}\right\rangle-\left\langle A\left(x^{n_{k}}-x^{n_{k}-1}\right), y^{n_{k}}-y^{*}\right\rangle \\
\leq \frac{\left\|y^{*}-y^{n_{k}}\right\|^{2}}{2 \sigma}+\frac{\left\|x^{*}-x^{n_{k}}\right\|^{2}}{2 \tau}
\end{gathered}
$$

from which we easily deduce that $x^{N} \rightarrow x^{*}$ and $y^{N} \rightarrow y^{*}$ as $N \rightarrow \infty$.

\section{References}

[1] R. K. Ahuja, T. L. Magnanti, and J. B. Orlin. Network flows. Prentice Hall Inc., Englewood Cliffs, NJ, 1993. Theory, algorithms, and applications.

[2] G. Alberti, G. Bouchitté, and D. Dal Maso. The calibration method for the Mumford-Shah functional and f ree-discontinuity problems. Calc. Var. Partial Differential Equations, 16(3):299-333, 2003.

[3] W. K. Allard. Total variation regularization for image denoising, I. Geometric th eory. SIAM J. Math. Anal., 39(4):1150-1190, 2007.

[4] F. Alter, V. Caselles, and A. Chambolle. A characterization of convex calibrable sets in $\mathbb{R}^{N}$. Math. Ann., 332(2):329-366, 2005. 
[5] F. Alter, V. Caselles, and A. Chambolle. Evolution of characteristic functions of convex sets in the plane by the minimizing total variation flow. Interfaces Free Bound., 7(1):29-53, 2005.

[6] L. Ambrosio. Corso introduttivo alla teoria geometrica della misura ed alle superfici minime. Appunti dei Corsi Tenuti da Docenti della Scuola. [Notes of Courses Given by Teachers at the School]. Scuola Normale Superiore, Pisa, 1997.

[7] L. Ambrosio, N. Fusco, and D. Pallara. Functions of bounded variation and free discontinuity problems. The Clarendon Press Oxford University Press, New York, 2000.

[8] L. Ambrosio and V. M. Tortorelli. Approximation of functionals depending on jumps by elliptic functionals via $\Gamma$-convergence. Comm. Pure Appl. Math., 43(8):999-1036, 1990.

[9] L. Ambrosio and V. M. Tortorelli. On the approximation of free discontinuity problems. Boll. Un. Mat. Ital. B (7), 6(1):105-123, 1992.

[10] B. Appleton and H. Talbot. Globally optimal geodesic active contours. $J$. Math. Imaging Vision, 23(1):67-86, 2005.

[11] K. J. Arrow, L. Hurwicz, and H. Uzawa. Studies in linear and non-linear programming. With contributions by H. B. Chenery, S. M. Johnson, S. Karlin, T. Marschak, R. M. Solow. Stanford Mathematical Studies in the Social Sciences, vol. II. Stanford University Press, Stanford, Calif., 1958.

[12] J.-F. Aujol, G. Gilboa, T. Chan, and S. Osher. Structure-texture image decomposition-modeling, algorithms, and parameter selection. Int. J. Comput. Vis., 67(1):111-136, 2006.

[13] A. Beck and M. Teboulle. A fast iterative shrinkage-thresholding algorithm for linear inverse problems. SIAM J. Imaging Sci., 2(1):183-202, 2009.

[14] G. Bellettini, M. Paolini, and C. Verdi. Convex approximations of functionals with curvature. Atti Accad. Naz. Lincei Cl. Sci. Fis. Mat. Natur. Rend. Lincei (9) Mat. Appl., 2(4):297-306, 1991. 
[15] J. Bioucas-Dias and M. Figueiredo. A new TwIST: two-step iterative shrinkage / thresholding algorithms for image restoration. IEEE Trans. on Image Processing, 16:2992-3004, 2007.

[16] Y. Boykov and V. Kolmogorov. An experimental comparison of min-cut/maxflow algorithms for energy minimization in vision. IEEE Trans. Pattern Analysis and Machine Intelligence, 26(9):1124-1137, September 2004.

[17] J. P. Boyle and R. L. Dykstra. A method for finding projections onto the intersection of convex sets in Hilbert spaces. In Advances in order restricted statistical inference (Iowa City, Iowa, 1985), volume 37 of Lecture Notes in Statist., pages 28-47. Springer, Berlin, 1986.

[18] K. A. Brakke. Soap films and covering spaces. J. Geom. Anal., 5(4):445-514, 1995.

[19] H. Brézis. Opérateurs maximaux monotones et semi-groupes de contractions dans les espaces de Hilbert. North-Holland Publishing Co., Amsterdam, 1973. North-Holland Mathematics Studies, No. 5. Notas de Matemática (50).

[20] V. Caselles, A. Chambolle, and M. Novaga. The discontinuity set of solutions of the TV denoising problem and some extensions. Multiscale Model. Simul., 6(3):879-894, 2007.

[21] V. Caselles, A. Chambolle, and M. Novaga. Regularity for solutions of the total variation denoising problem. Technical report, Preprint CMAP, 2009.

[22] A. Chambolle, D. Cremers, and T. Pock. A convex approach for computing minimal partitions. Technical Report 649, CMAP, Ecole Polytechnique, France, 2008.

[23] A. Chambolle and J. Darbon. On total variation minimization and surface evolution using parametric maximum flows. Int J Comput Vis, 2009.

[24] A. Chambolle, S. Levine, and B. Lucier. Some variations on total variationbased image smoothing. Technical report, CMAP, Ecole Polytechnique, 2009.

[25] T. F. Chan and S. Esedoḡlu. Aspects of total variation regularized $L^{1}$ function approximation. SIAM J. Appl. Math., 65(5):1817-1837 (electronic), 2005. 
[26] T. F. Chan, S. Esedoḡlu, and M. Nikolova. Algorithms for finding global minimizers of image segmentation and denoising models. SIAM J. Appl. Math., 66(5):1632-1648 (electronic), 2006.

[27] P. Combettes and V. Wajs. Signal recovery by proximal forward-backward splitting. SIAM Multiscale Modelling and Simulation, 2006.

[28] G. Dal Maso. Integral representation on $B V(\Omega)$ of $\Gamma$-limits of variational integrals. Manuscr. Math., 30(4):387-416, december 1979.

[29] G. David. Global minimizers of the Mumford-Shah functional. In Current developments in mathematics, 1997 (Cambridge, MA), pages 219-224. Int. Press, Boston, MA, 1999.

[30] J. Eckstein and D. Bertsekas. On the douglas-rachford splitting method and the proximal point algorithm for maximal monotone operators. Mathematical Programming, 55, 1992.

[31] I. Ekeland and R. Témam. Convex analysis and variational problems, volume 28 of Classics in Applied Mathematics. Society for Industrial and Applied Mathematics (SIAM), Philadelphia, PA, english edition, 1999. Translated from the French.

[32] E. Esser. Applications of lagrangian-based alternating direction methods and connections to split bregman. CAM Reports 09-31, UCLA, Center for Applied Math., 2009.

[33] E. Esser, X. Zhang, and T. Chan. A general framework for a class of first order primal-dual algorithms for tv minimization. CAM Reports 09-67, UCLA, Center for Applied Math., 2009.

[34] L. C. Evans and R. F. Gariepy. Measure theory and fine properties of functions. CRC Press, Boca Raton, FL, 1992.

[35] K. J. Falconer. The geometry of fractal sets, volume 85 of Cambridge Tracts in Mathematics. Cambridge University Press, Cambridge, 1986.

[36] H. Federer. Geometric measure theory. Springer-Verlag New York Inc., New York, 1969. 
[37] S. Geman and D. Geman. Stochastic relaxation, Gibbs distributions, and the Bayesian restoration of images. IEEE Trans. PAMI, PAMI-6(6):721-741, November 1984.

[38] M. Giaquinta, G. Modica, and J. Souček. Cartesian currents in the calculus of variations. I, volume 37 of Ergebnisse der Mathematik und ihrer Grenzgebiete. Springer-Verlag, Berlin, 1998.

[39] M. Giaquinta, G. Modica, and J. Souček. Cartesian currents in the calculus of variations. II, volume 38 of Ergebnisse der Mathematik und ihrer Grenzgebiete. Springer-Verlag, Berlin, 1998.

[40] E. Giusti. Minimal surfaces and functions of bounded variation. Birkhäuser Verlag, Basel, 1984.

[41] D. S. Hochbaum. An efficient algorithm for image segmentation, Markov random fields and related problems. J. ACM, 48(4):686-701 (electronic), 2001.

[42] B. Kawohl and T. Lachand-Robert. Characterization of Cheeger sets for convex subsets of the plane. Pacific J. Math., 225(1):103-118, 2006.

[43] V. Kolmogorov and R. Zabih. What energy functions can be minimized via graph cuts? IEEE Trans. Pattern Analysis and Machine Intelligence, 2(26):147-159, 2004.

[44] G. M. Korpelevich. Extrapolational gradient methods and their connection with modified Lagrangians. Ehkon. Mat. Metody, 19:694-703, 1983.

[45] M.-J. Lai, B. J. Lucier, and J. Wang. The convergence of a central-difference discretization of rudin-osher-fatemi model for image denoising. In Scale Space and Variational Methods in Computer Vision, volume 5567 of Lecture Notes in Computer Science, pages 514-526. Springer, 2009.

[46] G. Lawlor and F. Morgan. Paired calibrations applied to soap films, immiscible fluids, and surfaces or networks minimizing other norms. Pacific J. Math., 166(1):55-83, 1994.

[47] J. Lellmann, J. Kappes, J. Yuan, F. Becker, and C. Schnörr. Convex multiclass image labeling by simplex-constrained total variation. In Scale Space and Variational Methods in Computer Vision, volume 5567 of Lecture Notes in Computer Science, pages 150-162. Springer, 2009. 
[48] P.-L. Lions and B. Mercier. Splitting algorithms for the sum of two nonlinear operators. SIAM J. Numer. Anal., 16:964-979, 1979.

[49] Cécile Louchet. Modèles variationnels et bayésiens pour le débruitage d'images : de la variation totale vers les moyennes non-locales. $\mathrm{PhD}$ thesis, Université Paris-Descartes, 2008.

[50] B. J. Lucier and J. Wang. Error bounds for finite-difference methods for RudinOsher-Fatemi image smoothing. CAM Reports 09-70, UCLA, Center for Applied Math., 2009.

[51] F. Maddalena and S. Solimini. Lower semicontinuity properties of functionals with free discontinuities. Arch. Ration. Mech. Anal., 159(4):273-294, 2001.

[52] Y. Meyer. Oscillating patterns in image processing and nonlinear evolution equations, volume 22 of University Lecture Series. American Mathematical Society, Providence, RI, 2001. The fifteenth Dean Jacqueline B. Lewis memorial lectures.

[53] N. Meyers and J. Serrin. $H=W$. Proc. Nat. Acad. Sci. USA, 51:1055-1056, 1964.

[54] J.-M. Morel and S. Solimini. Variational Methods in Image Segmentation. Birkhäuser, Boston, 1995.

[55] D. Mumford. Pattern theory: The mathematics of perception. In Proceedings of the International Congress of Mathematicians, volume 3, 2002.

[56] D. Mumford and J. Shah. Optimal approximation by piecewise smooth functions and associated variational problems. Comm. Pure Appl. Math., 42:577$685,1989$.

[57] A. Nemirovski. Prox-method with rate of convergence $O(1 / t)$ for variational inequalities with Lipschitz continuous monotone operators and smooth convexconcave saddle point problems. SIAM J. Optim., 15(1):229-251, 2004.

[58] Yu. E. Nesterov. A method for solving the convex programming problem with convergence rate $O\left(1 / k^{2}\right)$. Dokl. Akad. Nauk SSSR, 269(3):543-547, 1983.

[59] Yu. E. Nesterov. Introductory lectures on convex optimization, volume 87 of Applied Optimization. Kluwer Academic Publisher, 2004. 
[60] Yu. E. Nesterov. Smooth minimization of nonsmooth functions. Mathemetical programmind Series A, 103:127-152, 2005.

[61] M. Nikolova. A variational approach to remove outliers and impulse noise. $J$. Math. Image Vis., 20(1-2):99-120, 2004.

[62] J. C. Picard and H. D. Ratliff. Minimum cuts and related problems. Networks, $5(4): 357-370,1975$.

[63] T. Pock, D. Cremers, H. Bischof, and A. Chambolle. An algorithm for minimizing the Mumford-Shah functional. In ICCV Proceedings, LNCS. Springer, 2009.

[64] T. Pock, D. Cremers, H. Bischof, and A. Chambolle. Global solutions of variational models with convex regularization. (submitted), 2010.

[65] L. Popov. A modification of the arrow-hurwicz method for search of saddl e points. Mathematical Notes, 28(5):845-848, 1980.

[66] M. Protter, I. Yavneh, and M. Elad. Closed-form MMSE estimation for signal denoising under sparse representation modelling over a unitary dictionary. Technical report, Technion, Haifa, 2009.

[67] R. T. Rockafellar. Convex analysis. Princeton Landmarks in Mathematics. Princeton University Press, Princeton, NJ, 1997. Reprint of the 1970 original, Princeton Paperbacks.

[68] L. Rudin, S. J. Osher, and E. Fatemi. Nonlinear total variation based noise removal algorithms. Physica D., 60:259-268, 1992. [also in Experimental Mathematics: Computational Issues in Nonlinear Science (Proc. Los Alamo Conf. 1991)].

[69] L. Schwartz. Théorie des Distributions. Hermann, 1966. (2 volumes).

[70] R. E. Showalter. Monotone operators in Banach space and nonlinear partial differential equations, volume 49 of Mathematical Surveys and Monographs. American Mathematical Society, Providence, RI, 1997.

[71] C. R. Vogel and M. E. Oman. Iterative methods for total variation denoising. SIAM J. Sci. Comput, 17(1):227-238, 1996. Special issue on iterative methods in numerical linear algebra (Breckenridge, CO, 1994). 
[72] C. Zach, D. Gallup, J. M. Frahm, and M. Niethammer. Fast global labeling for real-time stereo using multiple plane sweeps. In Vision, Modeling, and Visualization 2008, pages 243-252. IOS Press, 2008.

[73] M. Zhu and T. Chan. An efficient primal-dual hybrid gradient algorithm for total variation image restoration. CAM Reports 08-34, UCLA, Center for Applied Math., 2008.

[74] W. P. Ziemer. Weakly differentiable functions. Springer-Verlag, New York, 1989. Sobolev spaces and functions of bounded variation. 